University of Louisville

ThinkIR: The University of Louisville's Institutional Repository

Electronic Theses and Dissertations

$8-2010$

\title{
Inference for time to event and sojourn time data under right censoring using reweighting approaches.
}

Jie Fan

University of Louisville

Follow this and additional works at: https://ir.library.louisville.edu/etd

\section{Recommended Citation}

Fan, Jie, "Inference for time to event and sojourn time data under right censoring using reweighting approaches." (2010). Electronic Theses and Dissertations. Paper 421.

https://doi.org/10.18297/etd/421

This Doctoral Dissertation is brought to you for free and open access by ThinkIR: The University of Louisville's Institutional Repository. It has been accepted for inclusion in Electronic Theses and Dissertations by an authorized administrator of ThinkIR: The University of Louisville's Institutional Repository. This title appears here courtesy of the author, who has retained all other copyrights. For more information, please contact thinkir@louisville.edu. 


\title{
INFERENCE FOR TIME TO EVENT AND SOJOURN TIME DATA UNDER RIGHT CENSORING USING REWEIGHTING APPROACHES
}

By

\author{
Jie Fan \\ MPA, University of Louisville, 2005 \\ MS, University of Louisville, 2007

\begin{abstract}
A Dissertation
Submitted to the Faculty of the

Graduate School of University of Louisville

in Partial Fulfillment of the Requirements

for the Degree of
\end{abstract}

Doctor of Philosophy

Department of Bioinformatics and Biostatistics

University of Louisville

Louisville, KY

Aug. 2010 
Copy right 2010 by Jie Fan

All rights reserved 



\section{INFERENCE FOR TIME TO EVENT DATA AND SOJOURN TIME DATA}

UNDER RIGHT CENSORING USING REWEIGHTING APPROACHES

By

Jie Fan

MPA, University of Louisville, 2005

MS, University of Louisville, 2007

A Dissertation Approved on

June 16,2010

by the following Dissertation Committee:

Dissertation Director 


\section{DEDICATION}

This dissertation is dedicated to my parents

Mr. Sheyong Fan and Mrs. Xiaoying Lu

and my wife

Dejuan Chen

who provide me invaluable support. 


\section{ACKNOWLEDGEMENTS}

I would like to thank Dr. Somnath Datta, my dissertation director, for his guidance and encouragement. His suggestions help me to enhance the quality of this research and to improve my skills to handle complicated issues in research. This research was supported in part by Dr. Datta's grant from the U.S. National Science Foundation, which help me to overcome financial difficulty under economic recession. I would also like to thank my other committee members, Dr. Guy Brock, Dr. Maiying Kong, Dr. John Myers, and Dr. Chenxi Wang, for their comments and assistance over the past two years. Finally, I would like to express my thanks to my wife, Dejuan, for her understanding and supporting my decision to pursue the doctoral degree. I would have never made it without her patience. 


\begin{abstract}
INFERENCE FOR TIME TO EVENT AND SOJOURN TIME DATA UNDER RIGHT CENSORING USING REWEIGHTING APPROACHES
\end{abstract}

Jie Fan

June 16,2010

In this dissertation research, we aim to solve problems of two types of survival data, clustered survival data with potentially informative cluster size and sojourn time data. The methods for these two types of data are different. However, both data have right censored observations, and we use reweighting approaches to deal with the censoring issue.

In the first part of the dissertation research, we consider marginal AFT models for correlated survival data with potentially informative cluster size. Informative cluster size means that the size of the correlated groups may be predictive of their survival characteristics. Two competing proposals, cluster-weighted AFT (CWAFT) marginal model and non-cluster-weighted AFT (NCWAFT) marginal model, are investigated. Simulation and theoretical results show that the CWAFT approach produces unbiased parameter estimation, but that the NCWAFT model does not when the cluster size is informative. We use probability-probability plots to investigate statistical properties of confidence intervals and adopt Wald tests to examine power properties for the CWAFT model. To illustrate our analysis, we apply the CWAFT model to a dental study data set. 
In the second part of the dissertation research, we consider the problem of comparing sojourn time distributions of a transient state in a general multistate system in two samples (groups) when the transition times are right censored. Under this setup, the censoring induced on the weight times is complex since both the state entry and exit are subjected to right censoring. Using the reweighting principle, a two sample MannWhitney type U-statistic is constructed that compares only the uncensored state sojourn times from the two distributions. A second Mann-Whitney type statistic is also constructed using a different reweighting that allows for comparison when one of the two sojourn times is either uncensored or singly censored. While both statistics are asymptotically unbiased and reduce to the standard Mann-Whitney statistic when there is no censoring, the second statistic has smaller variance since it effectively uses larger pairs of samples. Asymptotic normality of these statistics are established. A test of comparing the equality of sojourn time distributions in two independent samples is constructed by symmetrizing the pair specific Mann-Whitney type statistics mentioned above. The testing methodology is illustrated using a kidney disease patients data set. 


\section{TABLE OF CONTENTS}

PAGE

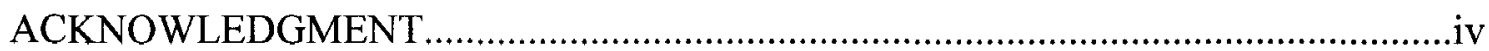

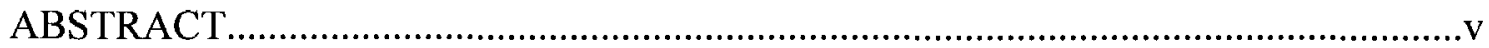

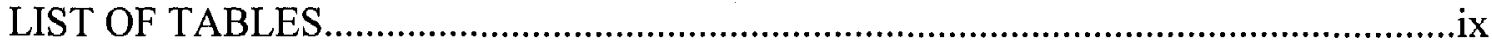

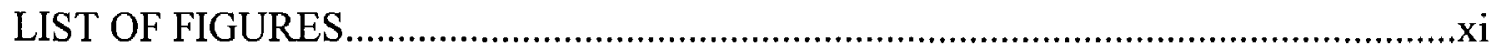

CHAPTER

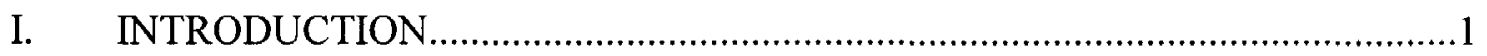

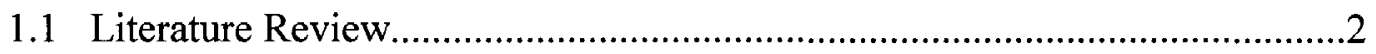

II. ACCELERATED FAILURE TIME MODELS FOR CLUSTERED SURVIVAL

DATA WITH POTENTIALLY INFORMATIVE CLUSTER SIZE........................11

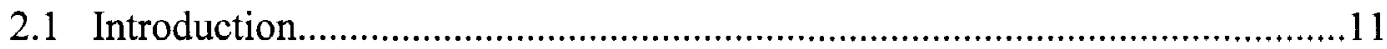

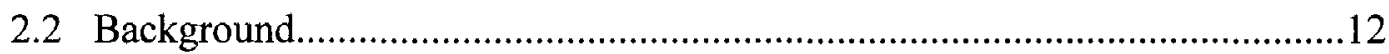

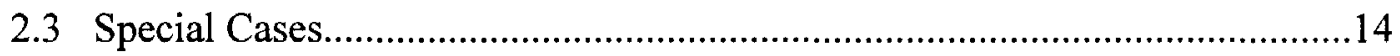

2.4 Proposed Competing AFT Models for Clustered Survival Data with Potentially Informative Cluster Size..............................................................27

2.5 A Mathematical Investigation of the Estimators Based on NCWAFT

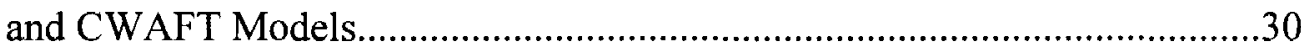

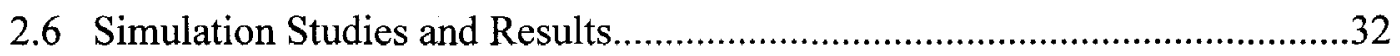

2.7 An Application to Dental Study Data............................................................

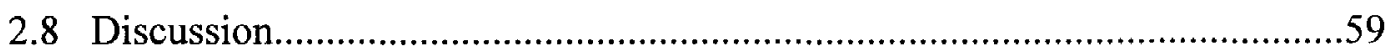

III. MANN-WHITNEY TEST FOR COMPARING SOJOURN TIME DISTRIBUTIONS WHEN TRANSITION TIMES ARE RIGHT CENSORED.....60

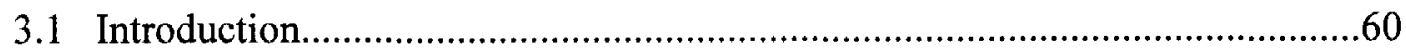




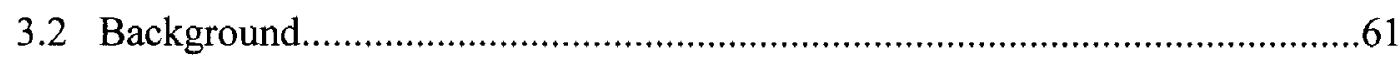

3.3 Mann-Whitney Type U-statistics for Sojourn Times in Presence of

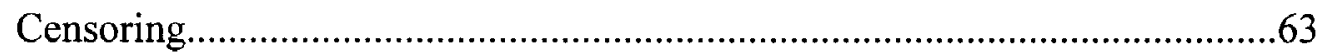

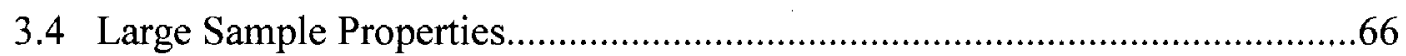

3.5 Testing the Equality of sojourn Time Distributions in 2 Groups......................69

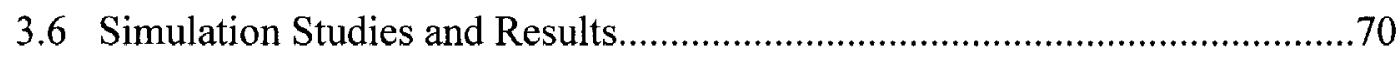

3.7 An Illustration Using Kidney Disease Data......................................................79

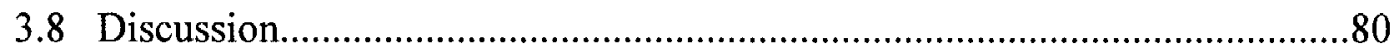

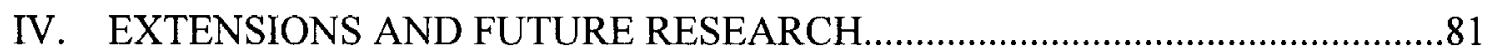

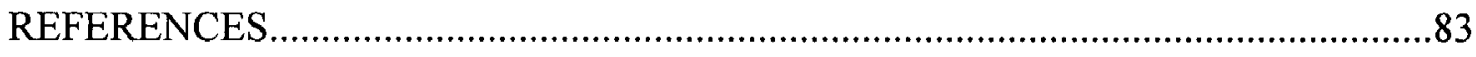

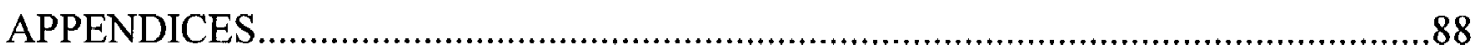

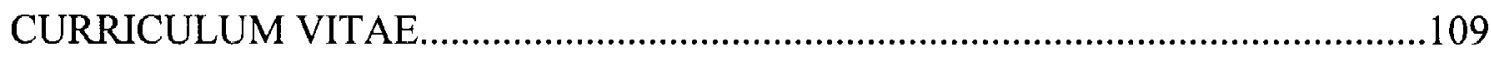




\section{LIST OF TABLE}

1. Independent survival data with random censoring time .18

2. Independent survival data with censoring times dependent on covariates.

3. Clustered survival data under potentially informative cluster size with random censoring times (simulation by equation (3))

4. Clustered survival data under potentially informative cluster size with random censoring times (simulation by equation (2))

5. Simulation results for the cluster-weighted AFT model when the cluster size is only related to the cluster effect (Betal $=0.3$ Beta $2=0.4$, Beta $3=-0.2$, Beta4 $=-0.3$, alpha $=0.25, \mathrm{r} 1=1.5, \mathrm{r} 2=3, \mathrm{z} 3=\mathrm{N}(1,0.15)$, error $=0.05)$.

6. Simulation results for the non-cluster-weighted AFT model when the cluster size is only related to the cluster effect (Betal $=0.3 \mathrm{Beta} 2=0.4$, Beta $3=-0.2$, Beta4 $=-0.3$, alpha $=0.25, \mathrm{r} 1=1.5, \mathrm{r} 2=3, \mathrm{z} 3=\mathrm{N}(1,0.15)$, error $=0.05)$

7. Simulation results for the cluster-weighted AFT model when the cluster size is only related to the cluster effect (Betal $=0.3$ Beta $2=0.4$, Beta $3=-0.2$, Beta4 $=-0.3$, alpha $=0.25, \mathrm{r} 1=1.5, \mathrm{r} 2=3, \mathrm{z} 3=\mathrm{N}(1,0.3)$, error $=0.05)$

8. Simulation results for the non-cluster-weighted AFT model when the cluster size is only related to the cluster effect (Betal $=0.3 \mathrm{Beta} 2=0.4$, Beta $3=-0.2$, Beta4 $=-0.3$, alpha $=0.25, \mathrm{r} 1=1.5, \mathrm{r} 2=3, \mathrm{z} 3=\mathrm{N}(1,0.3)$, error $=0.05)$

9. Simulation results for the cluster-weighted AFT model when the cluster size is only related to the cluster effect (Betal $=0.8$ Beta $2=1$, Beta $3=-0.75$, Beta4 $=-1.5$, alpha $=0.25, \mathrm{r} 1=1.5, \mathrm{r} 2=3, \mathrm{z} 3=\mathrm{N}(1,0.15)$, error $=0.05)$ 
10. Simulation results for the non-cluster-weighted AFT model when the cluster size is only related to the cluster effect (Beta1 $=0.8$ Beta2 $=1$, Beta3 $=-0.75$, Beta4 $=-1.5$, alpha $=0.25, \mathrm{r} 1=1.5, \mathrm{r} 2=3, \mathrm{z} 3=\mathrm{N}(1,0.15)$, error $=0.05)$.

11. Simulation results for the cluster-weighted AFT model when the cluster size is only related to the cluster effect (Beta1 $=0.3$ Beta $2=0.4$, Beta $3=-0.2$, Beta4 $=-0.3$, alpha $=0.25, \mathrm{r} 1=1.5, \mathrm{r} 2=3, \mathrm{z} 3=\mathrm{N}(1,0.15)$, error $=0.2)$.

12. Simulation results for the non-cluster-weighted AFT model when the cluster size is only related to the cluster effect (Beta $1=0.3$ Beta2 $=0.4$, Beta $3=-0.2$, Beta4 $=-0.3$, alpha $=0.25, \mathrm{rl}=1.5, \mathrm{r} 2=3, \mathrm{z} 3=\mathrm{N}(1,0.15)$, error $=0.2)$.

13. Simulation results for the cluster-weighted AFT model when the cluster size is related to both the cluster effect and the binary covariate ((Betal $=0.3$ Beta $2=0.4$, Beta $3=-0.2$, Beta $4=-0.3$, alpha $=0.25, \mathrm{r} 1=1.5, \mathrm{r} 2=4, \mathrm{r} 3=4$, $\mathrm{z} 3=\mathrm{N}(1,0.3)$,error $=0.05)$

14. Simulation results for the non-cluster-weighted AFT model when the cluster size is related to both the cluster effect and the binary covariate (Betal $=0.3$ Beta2 $=0.4$, Beta3 $=-0.2$, Beta4 $=-0.3$, alpha $=0.25, \mathrm{r} 1=1.5, \mathrm{r} 2=4, \mathrm{r} 3=-4$, $\mathrm{z} 3=\mathrm{N}(1,0.3)$,error $=0.05)$ 45

15. Simulation results for the cluster-weighted AFT model when the cluster size is related to both the cluster effect and the binary covariate (Betal $=0.3$ Beta2 $=0.4$, Beta $3=-0.2$, Beta $4=-0.3$, alpha $=0.15, \mathrm{r} 1=1.5, \mathrm{r} 2=4, \mathrm{r} 3=-4, \mathrm{z} 3=\mathrm{N}(1,0.15)$, error $=0.05$ )

16. Simulation results for the non-cluster-weighted AFT model when the cluster size is related to both the cluster effect and the binary covariate (Betal $=0.3$ Beta2 $=0.4$, Beta $3=-0.2$, Beta4 $=-0.3$, alpha $=0.15, \mathrm{r} 1=1.5, \mathrm{r} 2=4, \mathrm{r} 3=-4$, $\mathrm{z} 3=\mathrm{N}(1,0.15)$, error $=0.05)$.

17. Simulation results for the cluster-weighted AFT model when the cluster size is not informative $($ Beta $1=0.3 \mathrm{Beta} 2=0.4$, Beta $3=-0.2$, Beta $4=-0.3$, alpha $=0.25$, 
pai $=0.25, \mathrm{r} 1=1.5, \mathrm{r} 2=3, \mathrm{z3}=\mathrm{N}(1,0.15)$,error $=0.05)$

18. Simulation results for the non-cluster-weighted AFT model when the cluster size is not informative $($ Betal $=0.3$ Beta2 $=0.4$, Beta $3=-0.2$, Beta $4=-0.3$, alpha $=0.25$, pai $=0.25, \mathrm{r} 1=1.5, \mathrm{r} 2=3, \mathrm{z} 3=\mathrm{N}(1,0.15)$,error $=0.05)$ .54

19. Results for regression coefficients for the dental study. .58

20. Simulation results for $U$-statistics when censoring rates are same in two groups....72

21. Simulation results for $U$-statistics when censoring rates are different in two

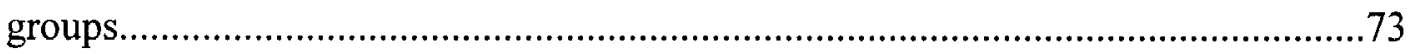

22. Simulation results for $U$-statistics under Markov model.........................................75

23. Simulation results for $U$-statistics when sojourn times and the state entry times are dependent 


\section{LIST OF FIGURES}

FIGURE

PAGE

1. Probability-Probability plots for parameters for two competing methods when the cluster size is only related to the cluster effect using the same parameters in Table 5-6

2. Probability-Probability plots for parameters for two competing methods when the cluster size is only related to the cluster effect using the same parameters in Table 7-8

3. Probability-Probability plots for parameters for two competing methods when the cluster size is only related to the cluster effect using the same parameters in Table 9-10

4. Probability-Probability plots for parameters for two competing methods when the cluster size is only related to the cluster effect using the same parameters in Table 11-12.

5. Probability-Probability plots for parameters for two competing methods when the cluster size is related to both the cluster effect and the covariate using the same parameters in Table 13-14

6. Probability-Probability plots for parameters for two competing methods when the cluster size is related to both the cluster effect and the covariate using the same parameters in Table 15-16. 
7. Probability-Probability plots for parameters for two competing methods when the cluster size is not informative using the same parameters in Table 17-18... 55

8. Power plot for $\beta_{1}$ when the cluster size is only related to the cluster effect.............57

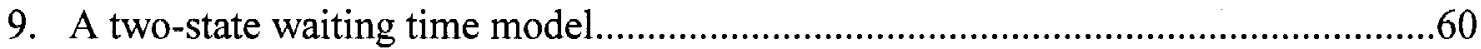

10. Power plot for proposed generalized Mann-Whitney method..............................78 


\section{CHAPTER I}

\section{INTRODUCTION}

Survival analysis is used to analyze time to event data. This type of analysis is also called "failure-time" analysis. Time to failure data arises in many areas, such as biology, demography, economics, engineering, epidemiology, medicine, and public health. Two most important characteristic features of survival data are non-negative survival time and possibly censored or truncated observations for some subjects.

The reasons for censored survival times include loss to follow up, failure to experience the event of interest by the end of the study, and failure or exit due to reasons other than the primary endpoint of interest. Truncation of survival data occurs when only subjects whose event time falls in a certain observational interval are observed. In contrast to censoring where partial information is available to investigator on each censored observation, a subject whose event time is not in the interval is not observed and no information is available on this subject.

The most common situation for survival data is right censoring, where the event time is known only to be greater than a certain time, but the exact survival time is unknown. In the first part of the dissertation research, we consider clustered survival data with potentially informative cluster size and, in the second part, we focus on two-stage sojourn time data. For each case, we are only interested in right censored data and use respective re-weighting method to deal with censoring observations. 


\subsection{Literature Review}

In this section, we discuss general overview of literature for methods relative to survival data.

\subsubsection{Analysis for Independent Identically Distributed Data without Censored Observations}

Generalized linear models (GLMs) are the standard methods for analyzing independent identically distributed (i.i.d.) data without missing value. The framework of GLMs is popularized by McCullagh and Nelder (1989). Dobon (2002) discussed the methods in detail for response and explanatory variables measured on various scales.

\subsubsection{Analysis for Clustered Data without Censored Observations}

Clustered data is data whose observations are not independently identical distributed but rather are collected in different clusters. In clustered data analysis, observations within each cluster are assumed correlated together, but observations between clusters are assumed independent. For example, we are interested in the weights of men aged 50 years selected from different states in USA. In this case, state is the cluster. Men from the same state share socio-economic and environmental conditions, and thus their weights are assumed somewhat correlated in the same state. The GEE method (Liang, Zeger, 1986) is a popular semi-parametric method that is used to analyze clustered data without censoring observation.

Longitudinal data is a special case of clustered data, where each subject is the cluster and is measured over multiple time periods. For example, we are interested in the weights of children in a class. Here, each child is a cluster, and his/her weight is measured repeatedly. Mixed-effects model is regarded as the standard statistical method to handle 
longitudinal data. However, in this dissertation research, our method is developed to address general clustered data rather than longitudinal data.

\subsubsection{Analysis for Clustered Data with Informative Cluster Size without Censored Observations}

For clustered data with informative cluster size, the outcomes of interest are associated with the cluster size. GEE, the popular method for clustered data, assumes non-informative cluster size, where the cluster size does not provide any information about the outcomes of interest. Informative cluster size often takes place in cluster-based design. The NHLBI Family Heart Study (NHLBI, 2009) is an example for this situation. The study analyzes genetic and non-genetic determinants of coronary heart disease, and cardiovascular risk factors.

For GEE method, each observation contributes equally in the estimating function due to non-informative cluster size assumption. However, when the cluster size is indeed informative, the GEE method leads to biased estimates because larger clusters tend to be overweighted, and smaller clusters tend to be underweighted.

Hoffman et al. (2001) proposed a within-cluster resampling (WCR) procedure, in which one observation is randomly sampled from each cluster. The observations in resampled data set are independent and thus the standard statistical methods can be applied. The final estimator is obtained by averaging over the estimates from the resampled data set, which are obtained by resampling the observed data with replacement many times. The WCR method is computationally extensive.

Follmann et al. (2003) established the asymptotic theories for the WCR approach and proposed a multiple outputation method that is a broad applications of the WCR method. Williamson et al. (2003) proposed a cluster weighted generalized estimated equation (CWGEE) where the estimating equation is inversely weighted by cluster sizes. Benhin et al. (2005) discussed in depth on a mean estimating approach, which is 
analogously similar to the work of Williamson et al. Wang et al. (2009) proposed a cluster weighted generalized estimating equation (CWGEE) for clustered longitudinal data and showed that CWGEE was superior to GEE when the cluster size was informative.

\subsubsection{Methods for Missing Data}

Imputation is one of popular approaches to deal with missing data. The basic idea of imputation is to fill in the missing data by using values under certain assumptions. Imputation can be single imputation and multiple imputation that replace each missing value with a set of possible values representing the uncertainty about the right value to impute. The appealing feature of imputation is that once the missing values are filled in, any statistical methods available for complete data can be applied.

LOCF, last observation carried forward, is a common practice of simple imputation for longitudinal data. This method includes every randomized subject who has at least one post treatment observation, and assumes that the missing values after patients' dropout are the same as the last value observed for that patient. Under this assumption, missing values are filled in without giving them within subject variability and the sample size is altered. This assumption of stability usually does not hold.

PMI, proper multiple imputation, are methods that use regression models to create more than one imputed data sets and thus variability within and between imputations. These methods assume that the missing data process can be fully captured by the regression model based on observed values. This assumption is called missing at random (MAR). MAR is less restrictive assumption than MCAR. Under MCAR assumption, missing data can not be dependent on either the observed or missing data. Under MAR assumption, missing data can be dependent on the observed data, but must be independent of the missing values that would have been observed. 
The partial imputation (PI), also called improved LOCF method, is a relatively new imputation method that is in-between the LOCF and PMI. PI does not always carry the observations to the last time-point of the study, but just far enough to balance the dropout patterns between the treatment groups. The fundamental principle is that when dropout pattern is similar between treatment groups, the relative comparison of the treatment effects will be less biased. PI has less imputation than LOCF, and thus is less biased since the assumption of stability usually does not hold.

Another popular approach for missing data is based on ranking observations. A large class of non-parametric methods is based on the ranks or scores of observations instead of the actual values including those popular in clinical trials such as Wilcoxon signed-rank test (1945) and Mann-Whitney test (1947). One can rank the missing values according to the reasons for withdraws, and, in longitudinal study cases, the time of dropout. For example, one can rank death the worst, followed by lack of efficacy, then adverse reaction, patient refusal, and so on. After missing data are replaced by their ranks, the usual testing procedure can be carried out. The major drawback of these methods is that they do not provide any estimation of treatment effect in the original measurement unit because the missing values are replaced by the ranks.

Reweighting approaches are proposed for survival data. Robins (1993) introduced a class of estimators using a data-reweighting method to estimate the marginal distribution for failure times. Satten et al. (2001) proposed an estimator of the marginal survival function of failure times that is in the class of survival function estimators proposed by Robins. Satten et al used the Aalen's additive hazard model for their reweighting scheme instead of the Cox proportional hazards model used by Robin (Aalen, 1980, 1989). Satten and Datta (2001) proposed a method to use the Kaplan-Meier estimator as an inverseprobability-of-censoring weighted average. Datta (2005) demonstrated three methods for estimation of the mean life for right censored data: area under the Kaplan-Meier curve, data imputation, and reweighting. 


\subsubsection{Analysis for Independent Identically Distributed Survival Data}

In survival analysis, the censoring time is usually assumed independent of the failure event time. The Product-Limit estimator (Kaplan and Meier, 1958), often called the Kaplan-Meier estimator, is the standard estimator of the survival function. The KaplanMeier estimator, a non-parametric method, provides consistent estimator of the survival function, but can not be used to test hypothesis such as no difference of survival functions between groups. The log-rank test is developed for this purpose. The proportional hazards model, also called the Cox model (Cox, 1972), is the most popular semi-parametric model in survival analysis. The partial likelihood theory of estimation used in the Cox model develops a flexible method for censored data. The proportional hazards model can be used to estimate survival time, test hypotheses, and predict survival time.

The accelerated failure time model (AFT) is an important alternative to the Cox model especially when the proportional hazards assumption does not hold. One obvious advantage of AFT model over Cox model is more intuitively interpretable. Koul et al. (1981) showed that their estimates are consistent and asymptotically normal under regularity conditions for AFT model with randomly right censored data. Wei et al. (1990) proposed methods for inference about a subset of the regression coefficients in the AFT model while regarding others as nuisance parameters for situation in which the distribution of the error term in the AFT model is unspecified.

There are also studies done for survival data with time-dependent covariates. Robins et al. (1992) introduced a class of semi-parametric accelerated failure time model, and derived a class of semi-parametric rank estimators. These estimates were also shown to be consistent, and asymptotically normal with consistently variances. Robins (1992) provided conditions to estimate the casual effect of a time-dependent treatment or exposure on time to an event of interest in the presence of time-dependent confounding covariates, and constructed estimators for the treatment effect under such conditions. 
Zeng and Lin (2007) proposed an approximate non-parametric maximum likelihood method for the accelerated failure time model with time dependent covariates, and estimated the regression parameters by maximizing a kernel-smoothed profile likelihood function.

\subsubsection{Analysis for Clustered Survival Data}

A basic assumption in the Cox model is independence of survival times given the current time and observed values of covariates. In another words, the Cox model assumes the studied population is homogenous, which means that all individuals in the study are under the same risk. However, in many situations, the study population should be considered to be heterogeneous rather than homogenous. Clustered survival times are the typical heterogeneous case encountered in many medical situations such as recurrent events on an individual or when the observations are clustered. A lot of studies on clustered survival data have been conducted. For example, McGilchrist and Aisbett (1991) did a study on recurrent times to inference for some kidney patients. In the study, the two recurrent times from the same patient are possibly to be correlated.

For analyzing clustered survival data, the most popular approaches are frailty modeling and marginal modeling. Generally speaking, frailty models are referred to models assuming the proportional hazards conditional on the frailty (cluster) for analyzing correlated or clustered failure time data, while the unconditional marginal distribution does not necessary follow the proportional hazards assumption.

Vaupel et al. (1979) introduced the term frailty in univariate survival models. The frailty is used to introduce random effect, association and unobserved heterogeneity into models for survival data. The fundamental idea is that individuals have different frailties, and that those who are most frail will experience failure earlier than the others.

Univariate frailty models were developed for situations in which the endpoint is a univariate survival time. A key issue for frailty models is the choice of the frailty 
distribution. Clayton (1978) proposed the gamma distribution for the frailty distribution, which is most popularly applied later. McGilchrist and Aisbett (1991) proposed the lognormal distribution. Aalen (1992) proposed the compound Poisson distribution.

The shared frailty models were developed for multivariate survival endpoints. In the share frailty models, individuals in a cluster are assumed to share the same frailty. It was introduced by Clayton (1978), and extensively studied in Hougaard (2000).

Wienke et al. (2003) discussed three limitations of the shared frailty models. First of all, the unobserved factors in these models are assumed to be the same within a cluster, which probably not reflect reality. Secondly, in shared frailty models, the dependence between survival times within a cluster is based on marginal distribution of survival times. However, the dependence parameter and the population heterogeneity can be confounded. Finally, the shared frailty, in most cases, only induces positive association within a cluster.

To avoid these limitations, the correlated frailty models are developed for multivariate failure time data. Yashin and Iachine (1995) proposed a correlated gamma frailty model with a bivariate survival distribution.

There has been some recent interest in a frailty model based on the accelerated failure time model for correlated failure time data. Lambert et al. (2004) discussed the AFT model with an additive frailty term and proposed a parametric method to estimate the parameters. Bayesian approach is widely adopted to estimate parameters for frailty model based on AFT model. Pan (2001) proposed to use frailties to account for possible correlations among failure times and to adapt an EM-like algorithm for parameter estimation. Zhang and Peng (2007) proposed a non-parametric estimation method based on M-estimators and the EM algorithm to estimate the parameters in the model.

The other approach, the unconditional marginal modeling approach, does not specify the dependence structure of the correlated failure times. Three methods for the marginal modeling approach include the proportional hazards model with a common 
baseline hazard function, different baseline hazard functions in the model, and parametric marginal model. Wei et al. (1989) proposed the marginal proportional hazards model for each failure type but the covariance matrix was estimated jointly across all failure types to adjust for the correlation. Lee et al. (1992) estimated the regression coefficients assuming independence among observations and provided a sandwich form of covariance matrix estimator. Spiekerman and Lin (1998) studied a more general marginal regression model for multivariate failure time data.

\subsubsection{Analysis for Clustered Survival Data with Potentially Informative Cluster Size}

Recently, the more complicated situation, clustered survival data with potentially informative size, is under investigation. Cong et al. (2007) generalized the marginal models of LEE et al. (1992) by incorporating the inverse of cluster sizes as weights into the score function to account for informative cluster sizes. Williamson et al. (2008) presented a method weighting two existing multivariate survival models by the inverse of the cluster size to deal with informative cluster size. The two existing models are a proportional hazards model with a common baseline hazard function denoted by LWA (Lee et al., 1992), and a parametric marginal model denoted by IWM or independent working model (Huster et al., 1989).

\subsubsection{Inference for Sojourn Times}

A multi-stage model is used to describe the time evolution of a system in which the series of events that an individual may experience are expressed as stages and occurrence of the events as transitions between the stages. Many problems with multivariate failure time or repeated events data can be defined in a multi-stage model. Multivariate failure time or repeated data are difficult to analyze when data have censored observations. Even if censoring is independent, one cannot calculate the Kaplan-Meier estimator of observed and censored waiting times in a multi-stage model unless the waiting times in each stage 
are independent. We call it semi-Markov assumption if the waiting times in each stage are assumed independent. Wang and Wells (1998) and Lin et al. (1999) proposed estimators in a three-stage network, assuming the hazard of being censored is the same in each stage. However, it is often implausible that individuals in different stages of a multistage model experience the same hazard for censoring. Satten and Datta (2002) proposed non-parametric estimates of the marginal cumulative distribution of stage waiting times and non-parametric estimates of marginal cumulative incidence function using right censored data from a multi-stage model. In their paper, they modeled the censoring hazard so that an estimate of integrated censoring hazard can be used in constructing the estimates of the waiting times distributions.

\subsubsection{A summary of the dissertation}

The rest of this dissertation is organized as follows. In Chapter II, we describe topics for accelerated failure models for clustered data with potentially informative class size including introduction, background, preliminary results, proposed competing AFT models with simulation studies and results, a mathematical investigation of the estimators based on NCWAFT and CWAF models, and an application to dental study data. Chapter III discusses Mann-Whitney test for comparing two sojourn time distributions when transition times are right censored. In this chapter, we cover introduction, background, generalized Mann-Whitney $U$-statistics for sojourn times under right censoring, asymptotic properties, testing the hypotheses, simulation studies and results, and an

illustration by kidney disease data. Chapter IV gives some concluding remarks and discusses future extensions and research for clustered survival data with potentially informative cluster size and sojourn time data. 


\section{CHAPTER II}

\section{ACCELERATED FAILURE TIME MODELS FOR CLUSTERED SURVIVAL DATA WITH POTENTIALLY INFORMATIVE CLUSTER SIZE}

\subsection{Introduction}

If there is no censored observation for a study in which the failure time $(T)$ is the response variable, and $X$ is the corresponding covariate vector, one may regress $T$ (or a suitable transformation of it) on $X$ directly through an additive error regression model. However, if there are censored observations in the data, the most popular approach is the proportional hazards model where the effect of $X$ is modeled through the hazard rate function of $T$; the model parameters are estimated using the partial likelihood function to obtain inferences about the covariate effect. Although the proportional hazards model is flexible and useful for testing whether a covariate affects survival, it is not easy to interpret the direct effect on event/survival times. The linear regression analysis with $h(\mathrm{~T})$ as the response variable is called the accelerated failure time model (AFT); the function $h$ is a known function of time taking values in the entire real line. The usual choice of $h$ is $\log$ function. The AFT model has gained increasing attention for survival analysis recently. 
Clustered data, also called multilevel data, often encountered in medical and biological studies where data are collected in different clusters. The typical feature of clustered data is that the measurements from the same cluster are usually correlated instead of independent. Also clustered data with informative cluster size are often encountered. Informative cluster size means that the distribution of the interested outcome in a given cluster depends on the size of the cluster. For example, in a quality of healthcare study, the duration of the hospital is probably related to the outcome measures of quality.

A key motivation of this research is to develop a flexible class of regression models that are similar in spirit with GLMs but retain the structure of the survival data. In this research, we consider two competing semi-parametric marginal AFT models, for right censored clustered survival data with potentially informative cluster size. These models are semi-parametric in the sense that the relationship of the response variable and covariates is parametric, but the random variances in the models are non-parametric. We will incorporate the cluster weight into the estimating equation to solve the challenge of the informative cluster size, and use the reweighting methods as in Satten et al. (2001) and Datta (2005) to deal with the censored observations.

\subsection{Background}

\subsubsection{Reweighting Methods}

As mentioned previously, reweighting is a method used to deal with missing data. The principle of mean unbiasedness through reweighting can be shown in the following procedure. Let $T^{*}$ be the true failure time, and $C$ be the censoring time. $T^{*}$ and $C$ are assumed independent. Let $T$ be the observed time with $T=\min \left(T^{*}, C\right)$ and $\delta$ be the censoring status indicator $\delta=I\left(T^{*} \leq C\right)$. Let $K_{c}$ be the weight function of the 
censoring variable with $K_{c}(t)=P(C>t), t \geq 0$. Note that under appropriate conditions

$$
\begin{gathered}
E\left\{\frac{h(T) \delta}{K_{c}(T-)}\right\}=E\left[E\left\{\frac{h\left(T^{*}\right) \delta}{K_{c}\left(T^{*}-\right)} \mid T^{*}\right\}\right] \\
=E\left[\frac{h\left(T^{*}\right)}{K_{c}\left(T^{*}-\right)} E\left\{I\left(C \geq T^{*}\right) \mid T^{*}\right\}\right]=E\left\{\frac{h\left(T^{*}\right)}{K_{c}\left(T^{*}-\right)} K_{c}\left(T^{*}-\right)\right\} \\
=E h\left(T^{*}\right) .
\end{gathered}
$$

Note that $E\left(I\left(C \geq T^{*}\right) \mid T^{*}\right)=K_{c}\left(T^{*}-\right)$ when the failure time $T^{*}$ and the censoring time $C$ are independent.

\subsubsection{Example: Calculation of $\boldsymbol{K}_{c}$}

As shown above, the Kaplan-Meier estimator for censoring times can serve as the inverse weight function to achieve unbiasedness by reweighting. We demonstrate below the calculation of $\boldsymbol{K}_{\boldsymbol{c}}$ by an artificial example with right censored observations:

$1,2,2+, 2+, 3,4+, 6,7$,

where a + indicates that the observation was right censored. Since we are calculating the Kaplan-Meier estimator (of the survival function) of the censoring times, we need to switch roles of failure and censoring times in the usual Kaplan-Meier calculation.

Kaplan-Meier estimator of censoring times

Time 2

4

Number of censored $2 \quad 1$

Number at risk

$6 \quad 3$

$\widehat{K}_{c}$

$1-\frac{2}{6}=\frac{2}{3} \quad \frac{2}{3}\left(1-\frac{1}{3}\right)=\frac{4}{9}$ 


\subsection{Special Cases}

Here, we develop the models starting with the simplest case of survival data without clustering. The models and the estimation methods will be extended leading to the full generalization considered in the next section.

\subsubsection{Notation for Independent Survival Data}

First consider the situation when the data for the individuals are independent of each other. Let $T_{i}^{*}$ be the possibly unobserved failure time for the $i$ th subject, and $C_{i}$ be the censoring time for $i$ th subject, $i$ from 1 to $n . T_{i}^{*}$ and $C_{i}$ are assumed independent. Let $T_{i}$ be the observed time for $i$ th subject with $T_{i}=\min \left(T_{i}^{*}, C_{i}\right)$ and $\delta_{i}$ be the indicator for $i$ th subject with $\delta_{i}=I\left(T_{i}^{*} \leq C_{i}\right)$.

The AFT model for independent survival data is

$$
Y_{i}:=\log T_{i}^{*}=\boldsymbol{Z}_{i}^{\prime} \boldsymbol{\beta}+\varepsilon_{i},
$$

where $\varepsilon_{i}$ is independent and identically distributed error with $E\left(\varepsilon_{i}\right)=0$, and $\operatorname{Var}\left(\varepsilon_{i}\right)=\sigma^{2} ; \boldsymbol{Z}_{i}$ denotes a $p$-vector of associated covariates for $i$ th subject and $\boldsymbol{\beta}$ is a $p$ vector of unknown parameters.

\subsubsection{Method of Parameter Estimation for Independent Survival Data}

We will show the way to get estimated parameters for independent survival data with censoring times dependent on covariates. For the AFT model, we use least squares method to estimate $\boldsymbol{\beta}$. To that end, we need to minimize

$$
\sum_{i=1}^{n}\left(Y_{i}-\boldsymbol{Z}_{i}^{\prime} \boldsymbol{\beta}\right)^{2}
$$


Taking derivative of $\sum_{i=1}^{n}\left(Y_{i}-\boldsymbol{Z}_{\boldsymbol{i}}^{\prime} \boldsymbol{\beta}\right)^{2}$ with respect to $\boldsymbol{\beta}$, and then setting it to 0 , we can obtain the estimating equation

$$
S(\boldsymbol{\beta}):=\sum_{i=1}^{n}\left(Y_{i}-\boldsymbol{Z}_{i}^{\prime} \boldsymbol{\beta}\right) \boldsymbol{Z}_{i}=0
$$

This estimating equation cannot be used directly to estimate the parameters because of censoring. We will build up the estimating equation for the censored data as follows. We assume that the censoring times $C_{i}$ are independent of the failure times $T_{i}^{*}$ given the covariates $\boldsymbol{Z}_{i}$. We then have

$$
E\left(\delta_{i} \mid \boldsymbol{Z}_{i}, T_{i}^{*}\right)=P\left(C_{i} \geq T_{i}^{*} \mid \boldsymbol{Z}_{i}, T_{i}^{*}\right)=K_{i, c}\left(T_{i}^{*}-\right),
$$

where we let $K_{i, c}(t)=P\left(C_{i} \geq t \mid \boldsymbol{Z}_{i}\right)=P\left(C_{i} \geq t \mid \boldsymbol{Z}_{i}, T_{i}^{*}\right)$ be the conditional survival function, or the inverse weight function, of the censoring time $C_{i}$ for $i$ th subject, given the covariates and the true failure times. Then,

$$
\begin{gathered}
E\left\{\frac{\delta_{i}}{K_{i, c}\left(T_{i}-\right)}\left(Y_{i}-\boldsymbol{Z}_{i}^{\prime} \boldsymbol{\beta}\right) \boldsymbol{Z}_{i}\right\}=E\left(E\left\{\frac{\delta_{i}}{K_{i, c}\left(T_{i}^{*}-\right)}\left(Y_{i}-\boldsymbol{Z}_{i}^{\prime} \boldsymbol{\beta}\right) \boldsymbol{Z}_{i} \mid \boldsymbol{Z}_{i}, T_{i}^{*}\right\}\right) \\
=E\left(\frac{1}{K_{i, c}\left(T_{i}^{*}-\right)}\left(Y_{i}-\boldsymbol{Z}_{i}^{\prime} \boldsymbol{\beta}\right) \boldsymbol{Z}_{i} E\left(\delta_{i} \mid \boldsymbol{Z}_{i}, T_{i}^{*}\right)\right) \\
=E\left(\frac{1}{K_{i, c}\left(T_{i}^{*}-\right)}\left(Y_{i}-\boldsymbol{Z}_{i}^{\prime} \boldsymbol{\beta}\right) \boldsymbol{Z}_{i} K_{i, c}\left(T_{i}^{*}-\right)\right) \\
=E\left(\left(Y_{i}-\boldsymbol{Z}_{i}^{\prime} \boldsymbol{\beta}\right) \boldsymbol{Z}_{i}\right)
\end{gathered}
$$

Therefore, an asymptotically unbiased estimating equation for the censored data is given by 


$$
S^{c}(\boldsymbol{\beta}):=\sum_{i=1}^{n} \frac{\delta_{i}\left(Y_{i}-\boldsymbol{Z}_{i}^{\prime} \boldsymbol{\beta}\right) \boldsymbol{Z}_{i}}{\widehat{K}_{i, c}\left(T_{i}-\right)}=0
$$

where $\hat{K}_{i, c}(t)$ is a consistent estimator of the inverse weight function $K_{i, c}(t)=P\left(C_{i} \geq t \mid \boldsymbol{Z}_{i}\right)$. Its construction will be discussed in detail in later sections.

Solving the equation, we can obtain the estimated parameters $\widehat{\boldsymbol{\beta}}$,

$$
\widehat{\boldsymbol{\beta}}=\left(\boldsymbol{Z}^{\prime} \boldsymbol{W} \boldsymbol{Z}\right)^{-1} \boldsymbol{Z}^{\prime} \boldsymbol{W} \boldsymbol{Y},
$$

where $\widehat{\boldsymbol{\beta}}$ is a $p$ by 1 vector, $\boldsymbol{Z}$ is a $n$ by $p$ matrix, $\boldsymbol{Y}$ is a $n$ by 1 vector, and $\boldsymbol{W}$ is a $n$ by $n$ diagonal matrix,

$$
\boldsymbol{W}=\left(\begin{array}{ccc}
\frac{\delta_{1}}{\widehat{K}_{i, c}\left(T_{1}-\right)} & \cdots & 0 \\
\vdots & \ddots & \vdots \\
0 & \cdots & \frac{\delta_{n}}{\widehat{K}_{i, c}\left(T_{n}-\right)}
\end{array}\right)
$$

\subsubsection{Modeling Censoring Times}

We consider two types of models for the censoring times.

\subsubsection{Independent Survival Data with Random Censoring Times}

The major difference in estimation between independent survival data with random censoring times and independent survival data with censoring times dependent on covariates is the way to estimate inverse weight function. Now, we consider the first case, in which we assume that the censoring times are not dependent on covariates. It is the simplest case in this research. If our proposed methods work for more complicate situations, then it must work for this simplest one. Therefore, we begin with this type of data, and then continue to more complicated data which we are really interested in. 


\section{Estimating inverse weight function}

Note that for the case of random censoring, the censoring hazard does not depend on the covariates and in such models $\widehat{K}_{i, c}(t)$ is constant in $i$, the estimated weight function can be computed by the standard Kaplan-Meier formula where the roles of failure and censoring times are switched.

More specifically, the weight function $K_{i, c}(t)=K_{c}(t)$ is estimated by

$$
\widehat{K}_{c}(t)=\prod_{\tau_{i} \leq t}\left[1-\frac{e_{i}}{Y_{i}}\right]
$$

where $\tau_{1}<\cdots<\tau_{d}$ are the distinct event times, $Y_{i}$ is the number of individuals at risk at time $\tau_{i}$, and $e_{i}$ is the number of censored events at time $\tau_{i}$. The example shown in section 2.3.2 is for random censoring situations.

\section{$\underline{\text { Simulation for Independent Survival Data with Random Censoring Times }}$}

Data are generated from the following model:

$$
Y_{i}=\log T_{i}^{*}=\beta_{1} Z_{i 1}+\beta_{2} Z_{i 2}+\varepsilon_{i},
$$

where $\beta_{1}=1, \beta_{2}=2$,

$$
Z_{i 1}=1, Z_{i 2}=\left\{\begin{array}{rr}
1 & \text { if } 1 \leq i \leq \frac{n}{2} \\
0 & \text { if } \frac{n}{2}+1 \leq i \leq n
\end{array},\right.
$$

and $\varepsilon_{i} \sim N(0,0.5)$.

The censoring times are assumed independent and identically distributed for each subject and were generated with a lognormal distribution. The log standard deviation was chosen as 1 for he lognormal distributions, and we varied log mean to control censoring rates approximately as $25 \%, 50 \%$ and $75 \%$. The simulation size of 1000 was used and the 
two group sizes were 30 and 200 respectively. The simulation results are reported in Table 1.

\section{Table 1}

Independent survival data with random censoring times.

\begin{tabular}{llrrr}
\hline Group size & & \multicolumn{3}{c}{ Censoring rate } \\
& & 0.25 & 0.5 & 0.75 \\
\hline 30 & $\operatorname{Bias}\left(\widehat{\beta}_{1}\right)$ & 0.004 & -0.002 & -0.056 \\
& $\operatorname{se}\left(\widehat{\beta}_{1}\right)$ & 0.133 & 0.154 & 0.216 \\
& $\operatorname{Bias}\left(\widehat{\beta}_{2}\right)$ & -0.029 & -0.089 & -0.235 \\
& $\operatorname{se}\left(\widehat{\beta}_{2}\right)$ & 0.214 & 0.301 & 0.438 \\
\multirow{2}{*}{200} & & & & \\
& $\operatorname{Bias}\left(\widehat{\beta}_{1}\right)$ & 0.002 & 0.001 & -0.001 \\
& $\operatorname{se}\left(\widehat{\beta}_{1}\right)$ & 0.051 & 0.059 & 0.095 \\
& $\operatorname{Bias}\left(\widehat{\beta}_{2}\right)$ & -0.005 & -0.020 & -0.149 \\
& $\operatorname{se}\left(\widehat{\beta}_{2}\right)$ & 0.081 & 0.117 & 0.226 \\
\hline
\end{tabular}

From Table 1, we find that the standard errors of the estimated parameters decrease when the sample size increases and the standard errors of the estimated parameters also decrease when the censoring rate decreases or the group size increases, which indicates positive sign for the method.

\subsubsection{Independent Survival Data with Censoring Times Dependent on Covariates}

In the previous case, the censoring times are generated completely at random. In other words, they do not depend on the covariates. Now, we will consider a case where the distribution of the censoring times depends on the covariates. It is easier to generate random censoring times and to estimate the inverse weight functions for the censoring times. For survival data with censoring times dependent on covariates we model 
censoring time by Aalen's linear hazard model and then use the method discussed in Satten et al. (2001) to calculate the inverse weight functions.

\section{Estimating cumulative hazard rate and inverse weight function}

For this type of survival data, we cannot estimate the inverse weight functions for censoring times by using the Product-Limit estimator for this situation. Instead, we use the following method to estimate the inverse weight functions as propose by Satten et al (2001).

Let $\lambda_{c}(t)$ denote the hazard function for censoring times, then Aalen's linear hazard model is defined as

$$
\lambda_{c}\left[t \mid \boldsymbol{Z}_{i}\right]=\sum_{k=1}^{p} \beta_{k}(t) Z_{i k}
$$

where $\beta_{k}(t), k=1, \ldots, p$, are an unknown parametric functions and $\boldsymbol{Z}_{i}=\left(Z_{i 1}, \ldots, Z_{i p}\right)$; we use the convention that $Z_{i 1}=1$ in order to include an intercept function.

Define cumulative risk function as $B_{k}(t)=\int_{0}^{t} \beta(s) d s, k=1, \ldots, p$. Aalen's model estimates $\boldsymbol{B}(t)=\left(B_{1}, \ldots, B_{p}\right)$ by:

$$
\widehat{\boldsymbol{B}}(t)=\sum_{i=1}^{n} I\left(T_{i} \leq t\right)\left(1-\delta_{i}\right) \boldsymbol{A}^{-1}\left(T_{i}\right) \boldsymbol{Z}_{i}
$$

where

$$
\boldsymbol{A}(t)=\sum_{i=1}^{n} I\left(T_{i} \geq t\right) \boldsymbol{Z}_{i} \boldsymbol{Z}_{i}^{T}
$$

The cumulative hazard for $t \leq T_{i}$ can be estimated by 


$$
\begin{gathered}
\widehat{\Lambda}_{c}\left[t \mid \boldsymbol{Z}_{i}\right]=\sum_{k=1}^{p} \int_{0}^{t} Z_{i k} d \widehat{B}_{k}(s) \\
=\sum_{i^{\prime}=1}^{n} I\left(T_{i^{\prime}} \leq t\right)\left(1-\delta_{i^{\prime}}\right) \boldsymbol{Z}_{i}^{T} \boldsymbol{A}^{-1}\left(T_{i^{\prime}}\right) \boldsymbol{Z}_{i^{\prime}} .
\end{gathered}
$$

The inverse weight function $K_{i, c}(t)$ is then estimated by:

$$
\widehat{K}_{i, c}(t)=\exp \left(-\widehat{\Lambda}_{c}\left[t \mid \boldsymbol{Z}_{i}\right]\right) .
$$

Simulation for Independent Survival Data with Censoring Times Dependent on

\section{Covariates}

Data are generated from the following model:

$$
Y_{i}=\log T_{i}^{*}=\beta_{1} Z_{i 1}+\beta_{2} Z_{i 2}+\beta_{3} Z_{i 3}+\varepsilon_{i}
$$

where $\beta_{1}=0.1, \beta_{2}=0.25, \beta_{3}=-0.3, Z_{i 1}=1, Z_{i 2} \sim \exp (22), Z_{i 3} \sim \exp (24)$ and $\varepsilon_{i} \sim N(0,0.15)$.

For this simulation, we generated the censoring times based on Aalen's linear model:

$$
\lambda_{\mathrm{c}}=b_{1} Z_{i 1}+b_{2} Z_{i 2}+b_{3} Z_{i 3},
$$

where the covariates, $Z_{i 1}, Z_{i 2}$ and $Z_{i 3}$, were the same with those for the model. Censoring times were obtained through the relationship among hazard rate, survival function and survival time. We varied the parameters, $b_{1}, b_{2}$ and $b_{3}$, to control the censoring rates as $25 \%, 50 \%$ and $75 \%$. The simulation size of 1000 was used and the two group sizes were 30 and 200 respectively. The simulation results are reported in Table 2. 
Table 2

Independent survival data with censoring times dependent on covariates.

\begin{tabular}{llrrr}
\hline Group size & & \multicolumn{3}{c}{ Censoring rate } \\
& & 0.25 & \multicolumn{1}{c}{0.5} & 0.75 \\
\hline 30 & $\operatorname{Bias}\left(\widehat{\beta}_{1}\right)$ & 0.012 & 0.023 & 0.028 \\
& $\operatorname{se}\left(\widehat{\beta}_{1}\right)$ & 0.046 & 0.059 & 0.113 \\
& $\operatorname{Bias}\left(\widehat{\beta}_{2}\right)$ & -0.016 & -0.051 & -0.214 \\
& $\operatorname{se}\left(\widehat{\beta}_{2}\right)$ & 0.598 & 0.783 & 1.948 \\
& $\operatorname{Bias}\left(\widehat{\beta}_{3}\right)$ & -0.032 & -0.019 & -0.032 \\
& $\operatorname{se}\left(\widehat{\beta}_{3}\right)$ & 0.647 & 0.844 & 1.929 \\
& & & & \\
& $\operatorname{Bias}\left(\widehat{\beta}_{1}\right)$ & 0.010 & 0.021 & 0.024 \\
& $\operatorname{se}\left(\widehat{\beta}_{1}\right)$ & 0.023 & 0.033 & 0.070 \\
& $\operatorname{Bias}\left(\widehat{\beta}_{2}\right)$ & -0.015 & -0.029 & -0.097 \\
& $\operatorname{se}\left(\widehat{\beta}_{2}\right)$ & 0.286 & 0.350 & 0.739 \\
$\operatorname{Bias}\left(\widehat{\beta}_{3}\right)$ & -0.012 & -0.027 & -0.071 \\
$\operatorname{se}\left(\widehat{\beta}_{3}\right)$ & 0.296 & 0.394 & 0.781 \\
\hline
\end{tabular}

In general, Table 2 shows the similar trend as table 1 . The bias for $\beta_{2}$ is bigger compared with biases of the other two parameters when the censoring rate is 0.75 .

\subsubsection{Notations for Clustered Survival Data}

For clustered survival data observations within a cluster are assumed to be dependent but observations between clusters are assumed to be independent. The cluster survival data can have two censoring time schemes including random censoring times and censoring times dependent on covariates. The following notation is used for clustered survival data under potentially informative cluster size.

Let $T_{i j}^{*}$ be the possibly unobserved failure time for the $j$ th subject within the $i$ th cluster, and $C_{i j}$ be the censoring time for the $j$ th subject within the $i$ th cluster, $1 \leq i \leq m$, and $1 \leq j \leq n_{i}$. Thus, $m$ denotes the total number of clusters and $n_{i}$ denotes 
the size of the $i$ th cluster. $T_{i j}^{*}$ and $C_{i j}$ are assumed independent, given the covariates $\boldsymbol{Z}_{i j}$. Let $T_{i j}$ be the observed time for the $j$ th subject within the $i$ th cluster with $T_{i j}=\min \left(T_{i j}^{*}, C_{i j}\right)$ and $\delta_{i j}$ be the indicator for the $j$ th subject within the $i$ th cluster with $\delta_{i j}=I\left(T_{i j}^{*} \leq C_{i j}\right)$.

An AFT model for clustered survival data under potentially informative cluster size is generated by

$$
Y_{i j}=\log T_{i j}^{*}=\boldsymbol{Z}_{i j}^{\prime} \boldsymbol{\beta}+\alpha_{i}+\varepsilon_{i j}
$$

where $\varepsilon_{i j}$ is independent and identically distributed error with $E\left(\varepsilon_{i j}\right)=0$, and $\operatorname{Var}\left(\varepsilon_{i j}\right)=\sigma_{1}^{2}, \alpha_{i}$ is independent and identically distributed random cluster effect with $E\left(\alpha_{i}\right)=0$ and $\operatorname{Var}\left(\alpha_{i}\right)=\sigma_{2}^{2}, \boldsymbol{Z}_{i j}$ denote a $p$-vector of associated covariates for the $j$ th subject within the $i$ th cluster and $\boldsymbol{\beta}$ is a $p$-vector of unknown parameters.

The informative cluster size has been generated as follows:

$$
n_{i}=1+n_{i}^{*} \text {, and } n_{i}^{*} \sim \operatorname{Pois}\left(\exp \left(g\left(\alpha_{i}\right)\right)\right)
$$

where $g\left(\alpha_{i}\right)=\gamma_{1}+\gamma_{2} \alpha_{i}$.

\subsubsection{Method of Parameter Estimation for Clustered Survival Data}

As for independent survival data, we use least square method to estimate $\boldsymbol{\beta}$ to fit the AFT model for clustered survival data. A working correlation matrix can be assumed for the failure times in a cluster to obtain parameter estimates. The simplest working correlation matrix is identity matrix, which corresponds to minimizing the unweighted sums of squares

$$
\sum_{i=1}^{m} \sum_{j=1}^{n_{i}}\left(Y_{i j}-\boldsymbol{Z}_{i j}^{\prime} \boldsymbol{\beta}\right)^{2}
$$

Equating the vector of first partial derivatives with zero we obtain the corresponding estimating equation 


$$
S(\boldsymbol{\beta})=\sum_{i=1}^{m} \sum_{j=1}^{n_{i}}\left(Y_{i j}-\boldsymbol{Z}_{i j}^{\prime} \boldsymbol{\beta}\right) \boldsymbol{Z}_{i j}=0
$$

As before, due to censoring, this equation can not be used. For each uncensored individual, or $\delta_{i j}=1$, the corresponding term has to be reweighted by the inverse of probability that it is censored leading to the censored data estimating equation:

$$
S^{c}(\boldsymbol{\beta})=\sum_{i=1}^{m} \sum_{j=1}^{n_{i}} \frac{\delta_{i j}\left(Y_{i j}-\boldsymbol{Z}_{i j}^{\prime} \boldsymbol{\beta}\right) \boldsymbol{Z}_{i j}}{K_{i j, c}\left(T_{i j}-\right)}=0 .
$$

The estimating equation (1) and its censored data version marginalize over all failure times regardless of their cluster memberships. This may not be appropriate for some applications where the primary sampling units are the clusters and we are interested in marginal relationships between the covariates and failure times per cluster. Since the larger clusters may influence the computation of parameter estimates to a greater extent, the inference may be biased. To compensate for this, we propose the inverse of the cluster size as weights in the estimating equation leading to the following modified (weighted) version of the censored data estimating equation:

$$
S_{W}^{c}(\boldsymbol{\beta})=\sum_{i=1}^{m} \frac{1}{n_{i}} \sum_{j=1}^{n_{i}} \frac{\delta_{i j}\left(Y_{i j}-\boldsymbol{Z}_{i j}^{\prime} \boldsymbol{\beta}\right) \boldsymbol{Z}_{i j}}{K_{i j, c}\left(T_{i j}-\right)}=0 .
$$

Arguing as in Williamson et al. (2003), we can see that this estimating equation is in line with the within cluster resampling principle of Hoffman et al. (2001).

In this research, we demonstrate that when the cluster size is informative of the relationship of failure times and covariates, the estimators obtained from (3) are (asymptotically) unbiased (and consistent), whereas the estimators obtained from (2) are biased and inconsistent. This is demonstrated later theoretically and in the simulation studies. We name the AFT model through by the equation (3) the cluster-weighted AFT 
(CWAFT) model and the non-cluster-weighted AFT (NCWAFT) model through the equation (2).

Solving (3), we can obtain the estimated parameters $\widehat{\boldsymbol{\beta}}$,

$$
\widehat{\boldsymbol{\beta}}=\left(\boldsymbol{Z}^{\prime} \boldsymbol{W}_{c l} \boldsymbol{Z}\right)^{-1} \boldsymbol{Z}^{\prime} \boldsymbol{W}_{\boldsymbol{c l}} \boldsymbol{Y},
$$

where $\widehat{\boldsymbol{\beta}}$ is a $p$ by 1 vector, $\boldsymbol{Z}$ is a $\sum_{i=1}^{m} n_{i}$ by $p$ matrix, $\boldsymbol{Y}$ is a $\sum_{i=1}^{m} n_{i}$ by 1 vector, and $\boldsymbol{W}_{\boldsymbol{c l}}$ is a $\sum_{i=1}^{m} n_{i}$ by $\sum_{i=1}^{m} n_{i}$ diagonal matrix,

$$
\boldsymbol{W}_{\boldsymbol{c l}}=\left(\begin{array}{ccccc}
\frac{\delta_{11}}{n_{1} \hat{K}_{i j, c}\left(T_{11}-\right)} & \cdots & \cdots & \cdots & 0 \\
\vdots & \ddots & & & \vdots \\
\vdots & & \frac{\delta_{1 n_{1}}}{n_{1} \hat{K}_{i j, c}\left(T_{i_{1}}-\right)} & & \vdots \\
\vdots & & & \ddots & \vdots \\
0 & \cdots & \cdots & \cdots & \frac{\delta_{m n_{m}}}{\left.n_{m} \hat{K}_{i j, c} T_{m n_{m}}-\right)}
\end{array}\right)
$$

\subsubsection{Modeling Clustered Censoring Times}

\subsubsection{Independent and Identically Distributed Censoring Times}

In this scenario, the censoring times are assumed to be independent and identically distributed. In other words, there is no clustering with respect to the censoring times. Also the censoring hazard is not influenced by the observed covariates in the regression model for the failure times.

\section{Estimating inverse weight function}

For clustered survival data with i.i.d. random censoring times, we need to pool the data (irrespective of their cluster membership), so that every subject at risk will be 
evaluated. Then, $K_{c}\left(T_{i j}\right)$ can be estimated by the same method for independent survival data with random censoring time.

\section{$\underline{\text { Simulation for Clustered Survival Data with Random Censoring Times }}$}

Data are generated from the following model:

$$
Y_{i j}=\log T_{i j}^{*}=\beta_{1} Z_{i j 1}+\beta_{2} Z_{i j 2}+\alpha_{i}+\varepsilon_{i j},
$$

where $\beta_{1}=0.5, \beta_{2}=0.8, Z_{i j 1}=1, Z_{i j 2}=\left\{\begin{array}{rr}1 & \text { if } 1 \leq i \leq \frac{m}{2} \\ 0 & \text { if } \frac{m}{2}+1 \leq i \leq m\end{array}\right.$

$\alpha_{i} \sim N(0,0.25), \varepsilon_{i j} \sim N(0,0.15)$, and $m$ is the number of the clusters.

We chose $g\left(\alpha_{i}\right)=4 \alpha_{i}$ to generate the informative cluster size. The censoring times are assumed independent and identically distributed for each subject and were generated with a lognormal distribution. The log standard deviation was chose as 1 for he lognormal distributions, and we varied log mean to control censoring rates approximately as $25 \%$, $50 \%$ and $75 \%$. The simulation size again was 1000 . The numbers of clusters, $m$, were chosen as 30 and 200 respectively, and the cluster size was random. The simulation results are in Table 3 and Table 4. The results in Table 3 were obtained by the CWAFT model, or equation (3), and the results in Table 4 were obtained by the NCWAFT model or equation (2). 
Table 3

Clustered survival data under potentially informative cluster size with random censoring times (Estimation by CWAFT model).

\begin{tabular}{llrrr}
\hline Number of cluster & & \multicolumn{3}{c}{ Censoring rate } \\
& & 0.25 & 0.5 & \multicolumn{1}{c}{0.75} \\
\hline 30 & $\operatorname{Bias}\left(\widehat{\beta}_{1}\right)$ & 0.001 & 0.001 & -0.017 \\
& $\operatorname{se}\left(\widehat{\beta}_{1}\right)$ & 0.121 & 0.144 & 0.191 \\
& $\operatorname{Bias}\left(\widehat{\beta}_{2}\right)$ & -0.018 & -0.031 & -0.050 \\
& $\operatorname{se}\left(\widehat{\beta}_{2}\right)$ & 0.183 & 0.229 & 0.329 \\
200 & $\operatorname{Bias}\left(\widehat{\beta}_{1}\right)$ & $<0.001$ & $<-0.001$ & -0.004 \\
& $\operatorname{se}\left(\widehat{\beta}_{1}\right)$ & 0.051 & 0.062 & 0.086 \\
& $\operatorname{Bias}\left(\widehat{\beta}_{2}\right)$ & -0.001 & -0.005 & -0.013 \\
& $\operatorname{se}\left(\widehat{\beta}_{2}\right)$ & 0.078 & 0.103 & 0.152 \\
\hline
\end{tabular}

The results in Tables 3 and Table 4 share the simulated data. We compare the results in Table 3 and Table 4 to see if the CWAFT model can perform better than NCWAFT model in presence of informative cluster size. From the results in Table 3, we find that the standard errors decrease when either the number of cluster increases or the censoring rate decreases, and that biases of all parameters are reasonably small for all situations. 


\section{Table 4}

Clustered survival data under potentially informative cluster size with random censoring times (Estimation by CWAFT model).

\begin{tabular}{llrrr}
\hline Number of cluster & & \multicolumn{3}{c}{ Censoring rate } \\
& & 0.25 & \multicolumn{1}{c}{0.5} & \multicolumn{1}{c}{0.75} \\
\hline 30 & $\operatorname{Bias}\left(\widehat{\beta}_{1}\right)$ & 0.134 & 0.131 & 0.111 \\
& $\operatorname{se}\left(\widehat{\beta}_{1}\right)$ & 0.137 & 0.160 & 0.206 \\
& $\operatorname{Bias}\left(\widehat{\beta}_{2}\right)$ & -0.016 & -0.030 & -0.058 \\
& $\operatorname{se}\left(\widehat{\beta}_{2}\right)$ & 0.199 & 0.243 & 0.339 \\
200 & $\operatorname{Bias}\left(\widehat{\beta}_{1}\right)$ & 0.154 & 0.154 & 0.149 \\
& $\operatorname{se}\left(\widehat{\beta}_{1}\right)$ & 0.062 & 0.073 & 0.101 \\
& $\operatorname{Bias}\left(\widehat{\beta}_{2}\right)$ & -0.007 & -0.009 & -0.016 \\
& $\operatorname{se}\left(\widehat{\beta}_{2}\right)$ & 0.090 & 0.114 & 0.163 \\
\hline
\end{tabular}

In Table 4, although the standard errors decrease when either the number of cluster increases or the censoring rate decreases, the biases of $\beta_{1}$ are too large for a good estimation for all the censoring rates and both the numbers of cluster. We can conclude from the above preliminary results that CWAFT model stands a good chance of working for clustered survival data with potentially informative cluster size.

\subsection{Proposed Competing AFT Models for Clustered Survival Data with Potentially Informative Cluster Size}

\subsubsection{Clustered Survival Data with Censoring Times Dependent on Covariates}

In the last case, we investigate the competing models for clustered survival data with potentially informative cluster size. The censoring times are independent of covariates in that scenario. Now, we consider clustered survival data with censoring times dependent on covariates. This type of data is what we are really interested in for this dissertation 
research. The equations (2) and (3) are still used for the NCWAFT model and the CWAFT model respectively. However, we should use different inverse weight functions.

\section{Estimating cumulative hazard rate and inverse weight function}

For clustered survival data with censoring times dependent on covariates, we need to pool and align the data and then use the same method for independent survival data with censoring times dependent on covariates to obtain the inverse weight function.

Let $\lambda_{c}(t)$ denote the hazard function for censoring times, then Aalen's linear hazard model is defined as:

$$
\lambda_{c}\left[t \mid \mathbf{Z}_{i j}\right]=\sum_{\mathrm{k}=1}^{p} \beta_{k}(t) Z_{i j k},
$$

where $\beta_{k}(t), k=1, \ldots, p$, are an unknown parametric functions, and $Z_{i j 1}=1$.

Defining cumulative risk function $B_{k}(t)=\int_{0}^{t} \beta(s) d t, k=1, \ldots, p$. As before, Aalen's model estimates $\boldsymbol{B}(t)=\left(B_{1}, \ldots, B_{p}\right)$ by:

$$
\widehat{\boldsymbol{B}}(t)=\sum_{i=1}^{m} \sum_{j=1}^{n_{i}} I\left(t_{i j} \leq t\right)\left(1-\delta_{i j}\right) \boldsymbol{A}^{-1}\left(t_{i j}\right) \boldsymbol{Z}_{i j}
$$

where $\boldsymbol{A}(t)=\sum_{i=1}^{m} \sum_{j=1}^{n_{i}} I\left(T_{i j} \geq t\right) \boldsymbol{Z}_{i j} \boldsymbol{Z}_{i j}^{T}$, and $\boldsymbol{Z}_{i j}=\left(Z_{i j 1}, \ldots, Z_{i j p}\right)$.

The cumulative hazard for $t \leq T_{i j}$ can be estimated by

$$
\begin{aligned}
\widehat{\Lambda}_{c}\left[t \mid \boldsymbol{Z}_{i j}\right]= & \sum_{k=1}^{p} \int_{0}^{t} Z_{i j \mathrm{k}} d \widehat{B}_{k}(s) \\
& =\sum_{i^{\prime}=1}^{m} \sum_{j^{\prime}=1}^{n_{i}} I\left(T_{i^{\prime} j^{\prime}} \leq t\right)\left(1-\delta_{i^{\prime} j^{\prime}}\right) \boldsymbol{Z}_{i j}^{T} \boldsymbol{A}^{-1}\left(T_{i^{\prime} j^{\prime}}\right) \boldsymbol{Z}_{i^{\prime} j^{\prime}}
\end{aligned}
$$


The inverse weight function $K_{i j, c}(t)$ for censoring time is estimated by

$$
\widehat{K}_{i j, c}(t)=\exp \left(-\widehat{\Lambda}_{c}\left[t \mid \mathbf{Z}_{i j}\right]\right)
$$

Under suitable regularity conditions, the estimators for cluster-weighted AFT model (CWAFT) model is asymptotically normally distributed with 0 mean and variancecovariance $M$,

$$
\sqrt{m}(\widehat{\boldsymbol{\beta}}-\boldsymbol{\beta}) \sim A N(0, M),
$$

where $M$ has a sandwiching form $M=\Gamma^{-1} \Sigma \Gamma^{-1}$.

A consistent estimator for $M$ will be $\widehat{M}=\widehat{\Gamma}^{-1} \widehat{\Sigma} \widehat{\Gamma}^{-1}$,

where

$$
\widehat{\boldsymbol{\Sigma}}=\frac{1}{m-1} \sum_{i=1}^{m}\left(S_{i, W}^{c}(\widehat{\boldsymbol{\beta}})-\bar{S}_{W}^{c}(\widehat{\boldsymbol{\beta}})\right)\left(S_{i, W}^{c}(\widehat{\boldsymbol{\beta}})-\bar{S}_{W}^{c}(\widehat{\boldsymbol{\beta}})\right)^{T}
$$

with

$$
\bar{S}_{W}^{c}(\widehat{\boldsymbol{\beta}})=\frac{1}{m} S_{W}^{c}(\widehat{\boldsymbol{\beta}}), \text { and } S_{i, W}^{c}(\widehat{\boldsymbol{\beta}})=\frac{1}{n_{i}} \sum_{j=1}^{n_{i}} \frac{\delta_{i j}\left(Y_{i j}-\boldsymbol{Z}_{i j}^{\prime} \widehat{\boldsymbol{\beta}}\right) \boldsymbol{Z}_{i j}}{\widehat{K}_{i j, c}\left(T_{i j-}\right)}
$$

and

$$
\widehat{\Gamma}^{-1}=\frac{1}{m} S_{W}^{c^{\prime}}(\widehat{\boldsymbol{\beta}})=\left.\frac{1}{m} \sum_{i=1}^{m} \frac{\partial S_{i, W}^{c}(\boldsymbol{\beta})}{\partial \boldsymbol{\beta}}\right|_{\boldsymbol{\beta}=\widehat{\boldsymbol{\beta}}}=\frac{1}{m} \sum_{i=1}^{m} \frac{1}{n_{i}} \sum_{j=1}^{n_{i}} \frac{-\delta_{i j} \boldsymbol{Z}_{i j} \boldsymbol{Z}_{i j}^{\prime}}{\widehat{K}_{i j, c}\left(T_{i j-}\right)} .
$$

In next section we investigate the bias properties of estimators obtained from (2) and (3) when the cluster size is informative. 


\subsection{A Mathematical Investigation of the Estimators Based on NCWAFT and CWAFT Models}

In this section, we will investigate the bias properties of estimators base on NCWAFT $\left(\widehat{\beta}_{N C W A F T}\right)$ and CWAFT $\left(\widehat{\beta}_{C W A F T}\right)$ models when the cluster size is informative. The model we consider the model

$$
Y_{i j}=\boldsymbol{Z}_{i j}^{\prime} \boldsymbol{\beta}+\alpha_{i}+\varepsilon_{i j}
$$

as before, where $\alpha_{i}$ is a cluster specific random effect, $\boldsymbol{Z}_{i j}$ is a subject specific covariate vector and $\varepsilon_{i j}$ is a subject specific random error.

Extending the arguments in Wang et al. (2009), we will show that $\widehat{\beta}_{N C W A F T}$ is asymptotically biased while $\widehat{\beta}_{C W A F T}$ is asymptotically unbiased (and inconsistent) when the cluster size is related to the cluster effect $\alpha_{i}$. More precisely, suppose, $n_{i}=g\left(\alpha_{i}\right)$, for some function $g$ such that $E\left\{\alpha_{i} g\left(\alpha_{i}\right)\right\} \neq 0$. Note that by definition,

$$
\begin{aligned}
\widehat{\boldsymbol{\beta}}_{N C W A F T} & =\left(\boldsymbol{Z}^{\prime} \boldsymbol{W} \boldsymbol{Z}\right)^{-1} \boldsymbol{Z}^{\prime} \boldsymbol{W} \boldsymbol{Y} \\
& =\left(\sum_{i=1}^{m} \sum_{j=1}^{n_{i}} \boldsymbol{Z}_{i j} \boldsymbol{Z}_{i j}^{\prime} \frac{\delta_{i j}}{K_{i j, c}\left(T_{i j}-\right)}\right)^{-1}\left(\sum_{i=1}^{m} \sum_{j=1}^{n_{i}} \boldsymbol{Z}_{i j} Y_{i j} \frac{\delta_{i j}}{K_{i j, c}\left(T_{i j}-\right)}\right) .
\end{aligned}
$$

Hence, as $m \rightarrow \infty$, by laws of large numbers (under suitable regularity conditions),

$$
\begin{gathered}
\widehat{\boldsymbol{\beta}}_{N C W A F T} \approx\left\{E\left(\sum_{i=1 j=1}^{m} \sum_{i j}^{n_{i}} \boldsymbol{Z}_{i j} \boldsymbol{Z}_{i j}^{\prime} \frac{\delta_{i j}}{K_{i j, c}\left(T_{i j}-\right)}\right)\right\}^{-1}\left\{E\left(\sum_{i=1}^{m} \sum_{j=1}^{n_{i}} \boldsymbol{Z}_{i j} Y_{i j} \frac{\delta_{i j}}{K_{i j, c}\left(T_{i j}\right)}\right)\right\} \\
=\left\{E\left(\sum_{i=1}^{m} \sum_{j=1}^{n_{i}} \boldsymbol{Z}_{i j} \boldsymbol{Z}_{i j}^{\prime}\right)\right\}^{-1}\left\{E\left(\sum_{i=1}^{m} \sum_{j=1}^{n_{i}} \boldsymbol{Z}_{i j} Y_{i j}\right)\right\}
\end{gathered}
$$

where $A_{n} \approx B_{n}$ means $A_{n}-B_{n} \rightarrow 0$ in probability, as well as in $L_{1}$, 


$$
\begin{aligned}
& =\left\{E\left(\sum_{i=1}^{m} \sum_{j=1}^{n_{i}} \boldsymbol{Z}_{i j} \boldsymbol{Z}_{i j}^{\prime}\right)\right\}^{-1}\left\{E\left(\sum_{i=1}^{m} \sum_{j=1}^{n_{i}} \boldsymbol{Z}_{i j} \boldsymbol{Z}_{i j}^{\prime} \boldsymbol{\beta}+\sum_{i=1}^{m} \alpha_{i} \sum_{j=1}^{n_{i}} \boldsymbol{Z}_{i j}+\sum_{i=1}^{m} \sum_{j=1}^{n_{i}} \boldsymbol{Z}_{i j} \varepsilon_{i j}\right)\right\} \\
& =\boldsymbol{\beta}+\left\{E\left(\sum_{i=1}^{m} \sum_{j=1}^{n_{i}} \boldsymbol{Z}_{i j} \boldsymbol{Z}_{i j}^{\prime}\right)\right\}^{-1} E\left(\sum_{i=1}^{m} \alpha_{i} \sum_{j=1}^{n_{i}} \boldsymbol{Z}_{i j}\right) \\
& =\boldsymbol{\beta}+\left\{E\left(\sum_{i=1}^{m} n_{i} \boldsymbol{Z}_{i 1} \boldsymbol{Z}_{i 1}^{\prime}\right)\right\}^{-1} E\left(\sum_{i=1}^{m} \alpha_{i} n_{i} \boldsymbol{Z}_{i 1}\right),
\end{aligned}
$$

under the assumption that the covariate distribution is exchangeable within a cluster $i$ given the cluster size $n_{i}$,

$$
\left.=\boldsymbol{\beta}+\frac{E(\alpha g(\alpha))}{E(g(\alpha))}\left\{E\left(\sum_{i=1}^{m} \boldsymbol{Z}_{i 1}^{\prime} \boldsymbol{Z}_{i 1}\right)\right\}^{-1} E\left(\sum_{i=1}^{m} \boldsymbol{Z}_{i 1}\right)\right\}
$$

Since by assumption, $E(\alpha g(\alpha)) \neq 0$, this shows that the in probability (and $L_{1}$ ) limit of $\widehat{\beta}_{N C W A F T}$ is not equal to $\beta$.

We have shown that non-cluster weighted AFT model introduces bias in presence of informative cluster size. On the other hand, we now demonstrate that estimators by cluster weighted AFT model are unbiased by a similar calculation.

$$
\begin{aligned}
\widehat{\boldsymbol{\beta}}_{C W A F T} \approx\left\{E\left(\sum_{i=1}^{m} \sum_{j=1}^{n_{i}} \boldsymbol{Z}_{i j} \boldsymbol{Z}_{i j}^{\prime} \frac{\delta_{i j}}{n_{i} K_{i j, c}\left(T_{i j}-\right)}\right)\right\}^{-1}\left\{E\left(\sum_{i=1}^{m} \sum_{j=1}^{n_{i}} \boldsymbol{Z}_{i j} Y_{i j} \frac{\delta_{i j}}{n_{i} K_{i j, c}\left(T_{i j}-\right)}\right)\right\} \\
=\left\{E\left(\sum_{i=1}^{m} \frac{1}{n_{i}} \sum_{j=1}^{n_{i}} \boldsymbol{Z}_{i j} \boldsymbol{Z}_{i j}^{\prime}\right)\right\}^{-1}\left\{E\left(\sum_{i=1}^{m} \frac{1}{n_{i}} \sum_{j=1}^{n_{i}} \boldsymbol{Z}_{i j} Y_{i j}\right)\right\} \\
=\boldsymbol{\beta}+\left\{E\left(\sum_{i=1}^{m} \frac{1}{n_{i}} \sum_{j=1}^{n_{i}} \boldsymbol{Z}_{i j} \boldsymbol{Z}_{i j}^{\prime}\right)\right\}^{-1} E\left(\sum_{i=1}^{m} \alpha_{i} \frac{1}{n_{i}} \sum_{j=1}^{n_{i}} \boldsymbol{Z}_{i j}\right) \\
=\boldsymbol{\beta}+\left\{E\left(\sum_{i=1}^{m} \boldsymbol{Z}_{i 1} \boldsymbol{Z}_{i 1}^{\prime}\right)\right\}^{-1} E\left(\sum_{i=1}^{m} \alpha_{i} \boldsymbol{Z}_{i 1}\right),
\end{aligned}
$$




$$
=\boldsymbol{\beta}+\left\{E\left(\sum_{i=1}^{m} \boldsymbol{Z}_{i 1} \boldsymbol{Z}_{i 1}^{\prime}\right)\right\}^{-1} \sum_{i=1}^{m} E\left(\alpha_{i}\right) E\left(\boldsymbol{Z}_{i 1}\right)=\beta+\mathbf{0}=\boldsymbol{\beta} .
$$

In above cases, we consider the cluster size is only related to the cluster effect. The situation could be more complicated when the cluster size is related to both the cluster effect and one covariate. Under this scenario, we will have

$$
\mathrm{E}\left(\widehat{\boldsymbol{\beta}}_{N C W A F T}\right)=\boldsymbol{\beta}+\gamma+o(1)
$$

where the first two components of $\gamma$ might be non-zero. Thus, the estimator of the parameter associated with the covariate will be biased in addition to the intercept. The simulation results will verify this point in next section.

\subsection{Simulation Studies and Results}

In section 2.6.1, we conducted studies with simulated data to assess performances of two competing proposals by bias, standard error and empirical standard error. In section 2.6.2, we present hypotheses testing results for our proposed cluster-weighted AFT model. In simulation studies, we consider the following AFT model:

$$
Y_{i j}=\log T_{i j}^{*}=\beta_{1} Z_{i j 1}+\beta_{2} Z_{i j 2}+\beta_{3} Z_{i j 3}+\beta_{4} Z_{i j 4}+\alpha_{i}+\varepsilon_{i j}
$$

where $T_{i j}^{*}$ are possibly unobserved failure times, $\varepsilon_{i j} \sim N\left(0, \sigma_{1}^{2}\right), \alpha_{i} \sim N\left(0, \sigma_{2}^{2}\right)$, $Z_{i j 1}=1$

$Z_{i j 2}=\left\{\begin{array}{cc}1 & \text { if } 1 \leq i \leq \frac{m}{2} \\ 0 & \text { if } \frac{m}{2}+1 \leq i \leq m\end{array}, Z_{i j 3} \sim N\left(1, \sigma_{3}^{2}\right)\right.$, and $Z_{i j 4}=Z_{i j 2} * Z_{i j 3}$. 
Censoring times were generated by the Aalen's linear model:

$$
\lambda_{\mathrm{c}}=\mathrm{b}_{1} Z_{i j 1}+\mathrm{b}_{2} Z_{i j 2}+\mathrm{b}_{3} Z_{i j 3}+\mathrm{b}_{4} Z_{i j 4},
$$

\subsubsection{Parameter Estimation}

To compare the performances of the cluster-weighted AFT model with that of noncluster-weighted AFT model, we conducted a number of simulations by varying number of cluster, censoring rate, parameter values, error distribution, cluster effect distribution, informative cluster size, and covariate distribution. For each setup, the simulation size was $n=1000$.

i) We considered a small sample size $n=30$, and a large sample size $n=200$.

ii) To generate censoring time, we defined $\mathbf{b}=c \boldsymbol{\beta}$. Three censoring rates were chosen to represent low censoring rate (0.25), median censoring rate (0.5) and high censoring rate (0.75), by controlling $c$, while keeping $\boldsymbol{\beta}$ unchanged.

iii) We considered two sets of parameter values: $\beta_{1}=0.3, \beta_{2}=0.4, \beta_{3}=-0.2$, $\beta_{4}=-0.3 ;$ and $\beta_{1}=0.8, \beta_{2}=1, \beta_{3}=-0.75, \beta_{4}=-1.5$.

iv) Two distributions of the continuous covariate were considered: $Z_{i j 3} \sim N(1,0.15)$, and $Z_{i j 3} \sim N(1,0.3)$.

v) Two cluster effect distributions were selected: $\alpha_{i} \sim N(0,0.25)$, and $\alpha_{i} \sim N(0,0.15)$.

vi) Two types of random informative cluster sizes were considered. For the first scenario the cluster size was only related to cluster effect $\alpha_{i}$. Let $g\left(\alpha_{i}\right)=\gamma_{1}+\gamma_{2} \alpha_{i}$. The cluster sizes were randomly generated $n_{i}=1+n_{i}^{*}$, and $n_{i}^{*} \sim \operatorname{Pois}\left(\exp \left(g\left(\alpha_{i}\right)\right)\right)$. We chose two pairs of size controlling parameters: $\gamma_{1}=1.5, \gamma_{2}=3 ; \gamma_{1}=-1, \gamma_{2}=4$.

For the second scenario the cluster size was related to both cluster effect $\alpha_{i}$ and the cluster level covariate $Z_{i j 2}$. Let $g\left(\alpha_{i}\right)=\gamma_{1}+\gamma_{2} \alpha_{i}+\gamma_{3} Z_{i j 2}$. The cluster sizes were 
randomly generated $n_{i}=1+n_{i}^{*}$, and $n_{i}^{*} \sim \operatorname{Pois}\left(\exp \left(g\left(\alpha_{i}\right)\right)\right)$. We selected two pairs of size controlling parameters: $\gamma_{1}=1.5, \gamma_{2}=3$ and $\gamma_{3}=1 ; \gamma_{1}=1.5, \gamma_{1}=4$ and $\gamma_{3}=-4$. vii) Error distributions: signal variance was defined as $\sigma_{s}^{2}=\boldsymbol{\beta}^{T} \boldsymbol{\Sigma}_{z} \boldsymbol{\beta}$; noise variance was defined as $\sigma_{n}^{2}$, where $\varepsilon_{i j} \sim N\left(0, \sigma_{n}^{2}\right)$. Noise to signal ratio is $r=\frac{\sigma_{n}^{2}}{\sigma_{s}^{2}}$. We used two noise to signal ratio values, $r=1$, and $r=0.2$.

We present simulation results in Table 5-16 when cluster size is informative, and tables for other simulation setups are attached in Appendix. When the cluster effect $\alpha_{i}$ were distributed as $N(0,0.15)$, both methods work well, which means both methods are valid when the variance of cluster size is small. We present bias, standard deviation (e.sd), i.e., $s d=\sqrt{\frac{1}{n-1} \sum_{i=1}^{n}\left(\widehat{\theta}_{i}-\bar{\theta}\right)^{2}}$, and the square root of the average of the estimated variance $(s d)$, i.e., $s d=\sqrt{\frac{1}{n} \sum_{i=1}^{n} \widehat{V}\left(\widehat{\theta}_{i}\right)}$ for the cluster-weighted AFT model and non-cluster-weighted AFT model respectively in Table 5 and Table 6 , where the cluster size is only related to cluster effect $\alpha_{i}$. From the tables, it is obvious that the estimator for intercept, $\widehat{\beta}_{1}$, is biased by the NCWAFT model. However, the CWAFT model performs well for all of the four parameters. Table 7 and Table 8 report results for simulation designs similar to those in Table 5 and Table 6 except the variance for covariate $Z_{i j 3}$. We find the same conclusion for Table 7 and Table 8 as that for Table 5 and Table 6. In Table 9 and Table 10, the cluster size is still only related to cluster effect. However, we used the second set of parameters. We again conclude that the NCWAFT model introduce estimation bias for the intercept while the CWAFT model performed well for estimation for all parameters. In Table 11 and Table 12, we chose $\gamma_{1}=-1$ and $\gamma_{2}=4$. Same conclusion is found again. We also report the simulation results for the competing methods in Table 13 and Table 14 respectively when the cluster size is related to both cluster effect $\alpha_{i}$ and the cluster level covariate $Z_{i j 2}$. Under this scenario, the NCWAFT model introduces bias for both $\widehat{\beta}_{1}$ and $\widehat{\beta}_{2}$, but the CWAFT model remains consistent estimation. In Table 15 and Table 16, the cluster size is 
related to both cluster effect $\alpha_{i}$ and the cluster level covariate $Z_{i j 2}$. However, the variance for covariate $Z_{i j 3}$ has the different value with that in table 13 and Table 14. From Table 15 and Table 16, we find that the NCWAFT did not perform well for both $\widehat{\beta}_{1}$ and $\widehat{\beta}_{2}$, while the CWAFT model did. 


\section{Table 5}

Simulation results for the cluster-weighted AFT model when the cluster size is only related to the cluster effect $($ Beta1 $=0.3$ Beta2 $=0.4$, Beta $3=-0.2$, Beta $4=-0.3$, alpha $=0.25$,

$$
\mathrm{r} 1=1.5, \mathrm{r} 2=3, \mathrm{z} 3=\mathrm{N}(1,0.15) \text {, error }=0.05) \text {. }
$$

\begin{tabular}{llrrr}
\hline Number of cluster & & \multicolumn{3}{c}{ Censoring rate } \\
& & 0.25 & 0.5 & \multicolumn{1}{c}{0.75} \\
\hline 30 & $\operatorname{Bias}\left(\widehat{\beta}_{1}\right)$ & 0.014 & 0.039 & 0.046 \\
& $\operatorname{e.sd}\left(\widehat{\beta}_{1}\right)$ & 0.259 & 0.289 & 0.366 \\
& $\operatorname{sd}\left(\widehat{\beta}_{1}\right)$ & 0.232 & 0.249 & 0.311 \\
& $\operatorname{Bias}\left(\widehat{\beta}_{2}\right)$ & -0.025 & -0.056 & -0.051 \\
& $\operatorname{e} \operatorname{sd}\left(\widehat{\beta}_{2}\right)$ & 0.406 & 0.471 & 0.708 \\
& $\operatorname{sd}\left(\widehat{\beta}_{2}\right)$ & 0.351 & 0.406 & 0.559 \\
& $\operatorname{Bias}\left(\widehat{\beta}_{3}\right)$ & 0.006 & -0.01 & -0.009 \\
& $\operatorname{e} \operatorname{sd}\left(\widehat{\beta}_{3}\right)$ & 0.247 & 0.277 & 0.354 \\
& $\operatorname{sd}\left(\widehat{\beta}_{3}\right)$ & 0.221 & 0.239 & 0.302 \\
& $\operatorname{Bias}\left(\widehat{\beta}_{4}\right)$ & 0.016 & 0.039 & 0.028 \\
& $\operatorname{e.sd}\left(\widehat{\beta}_{4}\right)$ & 0.389 & 0.452 & 0.68 \\
& $\operatorname{sd}\left(\widehat{\beta}_{4}\right)$ & 0.335 & 0.389 & 0.539 \\
& & & \\
& $\operatorname{Bias}\left(\widehat{\beta}_{1}\right)$ & 0.018 & 0.043 & 0.063 \\
& $\operatorname{e.sd}\left(\widehat{\beta}_{1}\right)$ & 0.100 & 0.113 & 0.153 \\
& $\operatorname{sd}\left(\widehat{\beta}_{1}\right)$ & 0.099 & 0.110 & 0.145 \\
& $\operatorname{Bias}\left(\widehat{\beta}_{2}\right)$ & -0.033 & -0.041 & -0.059 \\
& $\operatorname{e.sd}\left(\widehat{\beta}_{2}\right)$ & 0.152 & 0.184 & 0.271 \\
& $\operatorname{sd}\left(\widehat{\beta}_{2}\right)$ & 0.151 & 0.181 & 0.253 \\
$\operatorname{Bias}\left(\widehat{\beta}_{3}\right)$ & 0.004 & -0.007 & -0.018 \\
& $\operatorname{e.sd}\left(\widehat{\beta}_{3}\right)$ & 0.095 & 0.107 & 0.146 \\
$\operatorname{sd}\left(\widehat{\beta}_{3}\right)$ & 0.094 & 0.105 & 0.139 \\
$\operatorname{Bias}\left(\widehat{\beta}_{4}\right)$ & 0.018 & 0.016 & 0.026 \\
& $\operatorname{e.sd}\left(\widehat{\beta}_{4}\right)$ & 0.144 & 0.176 & 0.257 \\
$\operatorname{sd}\left(\widehat{\beta}_{4}\right)$ & 0.144 & 0.173 & 0.243 \\
\hline & & &
\end{tabular}




\section{Table 6}

Simulation results for the non-cluster-weighted AFT model when the cluster size is only related to the cluster effect $($ Betal $=0.3$ Beta $2=0.4$, Beta $3=-0.2$, Beta $4=-0.3$, alpha $=0.25$,

$$
\mathrm{rl}=1.5, \mathrm{r} 2=3, \mathrm{z} 3=\mathrm{N}(1,0.15), \text { error }=0.05) \text {. }
$$

\begin{tabular}{|c|c|c|c|c|}
\hline \multirow[t]{2}{*}{ Number of cluster } & & \multicolumn{3}{|c|}{ Censoring rate } \\
\hline & & 0.25 & 0.5 & 0.75 \\
\hline \multirow[t]{12}{*}{30} & $\operatorname{Bias}\left(\widehat{\beta}_{1}\right)$ & 0.155 & 0.170 & 0.161 \\
\hline & $\operatorname{e.sd}\left(\widehat{\beta}_{1}\right)$ & 0.197 & 0.231 & 0.315 \\
\hline & $\operatorname{sd}\left(\widehat{\beta}_{1}\right)$ & 0.173 & 0.199 & 0.281 \\
\hline & $\operatorname{Bias}\left(\widehat{\beta}_{2}\right)$ & -0.023 & -0.055 & -0.052 \\
\hline & $\operatorname{e.sd}\left(\widehat{\beta}_{2}\right)$ & 0.309 & 0.403 & 0.684 \\
\hline & $\operatorname{sd}\left(\widehat{\beta}_{2}\right)$ & 0.275 & 0.347 & 0.511 \\
\hline & $\operatorname{Bias}\left(\widehat{\beta}_{3}\right)$ & 0.006 & -0.004 & 0.006 \\
\hline & e. $\operatorname{sd}\left(\widehat{\beta}_{3}\right)$ & 0.172 & 0.21 & 0.302 \\
\hline & $\operatorname{sd}\left(\widehat{\beta}_{3}\right)$ & 0.170 & 0.195 & 0.275 \\
\hline & $\operatorname{Bias}\left(\widehat{\beta}_{4}\right)$ & 0.014 & 0.039 & 0.023 \\
\hline & $\operatorname{e.sd}\left(\widehat{\beta}_{4}\right)$ & 0.279 & 0.375 & 0.649 \\
\hline & $\operatorname{sd}\left(\widehat{\beta}_{4}\right)$ & 0.267 & 0.335 & 0.493 \\
\hline \multirow[t]{12}{*}{200} & $\operatorname{Bias}\left(\widehat{\beta}_{1}\right)$ & 0.179 & 0.209 & 0.222 \\
\hline & $\operatorname{e.sd}\left(\widehat{\beta}_{1}\right)$ & 0.095 & 0.115 & 0.173 \\
\hline & $\operatorname{sd}\left(\widehat{\beta}_{1}\right)$ & 0.076 & 0.096 & 0.154 \\
\hline & $\operatorname{Bias}\left(\widehat{\beta}_{2}\right)$ & -0.033 & -0.05 & -0.069 \\
\hline & e.sd $\left(\widehat{\beta}_{2}\right)$ & 0.141 & 0.187 & 0.323 \\
\hline & $\operatorname{sd}\left(\widehat{\beta}_{2}\right)$ & 0.122 & 0.174 & 0.279 \\
\hline & $\operatorname{Bias}\left(\widehat{\beta}_{3}\right)$ & 0.006 & -0.005 & -0.006 \\
\hline & $\operatorname{e.sd}\left(\widehat{\beta}_{3}\right)$ & 0.081 & 0.102 & 0.163 \\
\hline & $\operatorname{sd}\left(\widehat{\beta}_{3}\right)$ & 0.075 & 0.094 & 0.151 \\
\hline & $\operatorname{Bias}\left(\widehat{\beta}_{4}\right)$ & 0.018 & 0.025 & 0.031 \\
\hline & e.sd $\left(\widehat{\beta}_{4}\right)$ & 0.125 & 0.176 & 0.304 \\
\hline & $\operatorname{sd}\left(\widehat{\beta}_{4}\right)$ & 0.119 & 0.167 & 0.269 \\
\hline
\end{tabular}


Table 7

Simulation results for the cluster-weighted AFT model when the cluster size is only related to the cluster effect $($ Beta $1=0.3$ Beta $2=0.4$, Beta $3=-0.2$, Beta $4=-0.3$, alpha $=0.25$,

$$
\mathrm{rl}=1.5, \mathrm{r} 2=3, \mathrm{z} 3=\mathrm{N}(1,0.3) \text {, error }=0.05) \text {. }
$$

\begin{tabular}{|c|c|c|c|c|}
\hline \multirow[t]{2}{*}{ Number of cluster } & & \multicolumn{3}{|c|}{ Censoring rate } \\
\hline & & 0.25 & 0.5 & 0.75 \\
\hline \multirow[t]{12}{*}{30} & $\operatorname{Bias}\left(\widehat{\beta}_{1}\right)$ & 0.028 & 0.045 & 0.049 \\
\hline & $\operatorname{e.sd}\left(\widehat{\beta}_{1}\right)$ & 0.149 & 0.166 & 0.207 \\
\hline & $\operatorname{sd}\left(\widehat{\beta}_{1}\right)$ & 0.133 & 0.143 & 0.172 \\
\hline & $\operatorname{Bias}\left(\widehat{\beta}_{2}\right)$ & -0.022 & -0.045 & -0.051 \\
\hline & $\operatorname{e.sd}\left(\widehat{\beta}_{2}\right)$ & 0.232 & 0.264 & 0.393 \\
\hline & $\operatorname{sd}\left(\widehat{\beta}_{2}\right)$ & 0.202 & 0.234 & 0.307 \\
\hline & $\operatorname{Bias}\left(\widehat{\beta}_{3}\right)$ & -0.001 & -0.007 & -0.006 \\
\hline & $\operatorname{e.sd}\left(\widehat{\beta}_{3}\right)$ & 0.125 & 0.142 & 0.185 \\
\hline & $\operatorname{sd}\left(\widehat{\beta}_{3}\right)$ & 0.113 & 0.123 & 0.154 \\
\hline & $\operatorname{Bias}\left(\widehat{\beta}_{4}\right)$ & 0.013 & 0.026 & 0.028 \\
\hline & $\operatorname{e.sd}\left(\widehat{\beta}_{4}\right)$ & 0.199 & 0.227 & 0.346 \\
\hline & $\operatorname{sd}\left(\widehat{\beta}_{4}\right)$ & 0.172 & 0.202 & 0.273 \\
\hline \multirow[t]{12}{*}{200} & $\operatorname{Bias}\left(\widehat{\beta}_{1}\right)$ & 0.029 & 0.057 & 0.070 \\
\hline & $\operatorname{e.sd}\left(\widehat{\beta}_{1}\right)$ & 0.059 & 0.069 & 0.088 \\
\hline & $\operatorname{sd}\left(\widehat{\beta}_{1}\right)$ & 0.056 & 0.065 & 0.083 \\
\hline & $\operatorname{Bias}\left(\widehat{\beta}_{2}\right)$ & -0.025 & -0.036 & -0.048 \\
\hline & $\operatorname{e.sd}\left(\widehat{\beta}_{2}\right)$ & 0.089 & 0.111 & 0.156 \\
\hline & $\operatorname{sd}\left(\widehat{\beta}_{2}\right)$ & 0.088 & 0.107 & 0.147 \\
\hline & $\operatorname{Bias}\left(\widehat{\beta}_{3}\right)$ & 0.001 & -0.011 & -0.017 \\
\hline & $\operatorname{e.sd}\left(\widehat{\beta}_{3}\right)$ & 0.049 & 0.057 & 0.075 \\
\hline & $\operatorname{sd}\left(\widehat{\beta}_{3}\right)$ & 0.048 & 0.055 & 0.073 \\
\hline & $\operatorname{Bias}\left(\widehat{\beta}_{4}\right)$ & 0.011 & 0.011 & 0.015 \\
\hline & $\operatorname{e.sd}\left(\widehat{\beta}_{4}\right)$ & 0.074 & 0.094 & 0.133 \\
\hline & $\operatorname{sd}\left(\widehat{\beta}_{4}\right)$ & 0.075 & 0.091 & 0.127 \\
\hline
\end{tabular}




\section{Table 8}

Simulation results for the non-cluster-weighted AFT model when the cluster size is only related to the cluster effect $($ Beta $1=0.3$ Beta $2=0.4$, Beta $3=-0.2$, Beta $4=-0.3$, alpha $=0.25$, $\mathrm{r} 1=1.5, \mathrm{r} 2=3, \mathrm{z} 3=\mathrm{N}(1,0.3)$, error $=0.05)$.

\begin{tabular}{|c|c|c|c|c|}
\hline \multirow[t]{2}{*}{ Number of cluster } & & \multicolumn{3}{|c|}{ Censoring rate } \\
\hline & & 0.25 & 0.5 & 0.75 \\
\hline \multirow[t]{12}{*}{30} & $\operatorname{Bias}\left(\widehat{\beta}_{1}\right)$ & 0.167 & 0.177 & 0.167 \\
\hline & $\operatorname{e.sd}\left(\widehat{\beta}_{1}\right)$ & 0.140 & 0.156 & 0.190 \\
\hline & $\operatorname{sd}\left(\widehat{\beta}_{1}\right)$ & 0.092 & 0.106 & 0.150 \\
\hline & $\operatorname{Bias}\left(\widehat{\beta}_{2}\right)$ & -0.020 & -0.046 & -0.066 \\
\hline & $\operatorname{e.sd}\left(\widehat{\beta}_{2}\right)$ & 0.206 & 0.235 & 0.345 \\
\hline & $\operatorname{sd}\left(\widehat{\beta}_{2}\right)$ & 0.151 & 0.192 & 0.280 \\
\hline & $\operatorname{Bias}\left(\widehat{\beta}_{3}\right)$ & 0.003 & -0.001 & 0.007 \\
\hline & $\operatorname{e.sd}\left(\widehat{\beta}_{3}\right)$ & 0.093 & 0.111 & 0.161 \\
\hline & $\operatorname{sd}\left(\widehat{\beta}_{3}\right)$ & 0.087 & 0.099 & 0.139 \\
\hline & $\operatorname{Bias}\left(\widehat{\beta}_{4}\right)$ & 0.010 & 0.027 & 0.035 \\
\hline & $\operatorname{e} . \operatorname{sd}\left(\widehat{\beta}_{4}\right)$ & 0.146 & 0.180 & 0.293 \\
\hline & $\operatorname{sd}\left(\widehat{\beta}_{4}\right)$ & 0.137 & 0.171 & 0.249 \\
\hline \multirow[t]{12}{*}{200} & $\operatorname{Bias}\left(\widehat{\beta}_{1}\right)$ & 0.197 & 0.230 & 0.234 \\
\hline & $\operatorname{e.sd}\left(\widehat{\beta}_{1}\right)$ & 0.071 & 0.089 & 0.112 \\
\hline & $\operatorname{sd}\left(\widehat{\beta}_{1}\right)$ & 0.043 & 0.056 & 0.086 \\
\hline & $\operatorname{Bias}\left(\widehat{\beta}_{2}\right)$ & -0.025 & -0.046 & -0.066 \\
\hline & e.sd $\left(\widehat{\beta}_{2}\right)$ & 0.101 & 0.129 & 0.189 \\
\hline & $\operatorname{sd}\left(\widehat{\beta}_{2}\right)$ & 0.072 & 0.103 & 0.159 \\
\hline & $\operatorname{Bias}\left(\widehat{\beta}_{3}\right)$ & -0.001 & -0.011 & -0.008 \\
\hline & $\operatorname{e.sd}\left(\widehat{\beta}_{3}\right)$ & 0.047 & 0.064 & 0.089 \\
\hline & $\operatorname{sd}\left(\widehat{\beta}_{3}\right)$ & 0.039 & 0.052 & 0.079 \\
\hline & $\operatorname{Bias}\left(\widehat{\beta}_{4}\right)$ & 0.012 & 0.018 & 0.025 \\
\hline & $\operatorname{e.sd}\left(\widehat{\beta}_{4}\right)$ & 0.069 & 0.099 & 0.157 \\
\hline & $\operatorname{sd}\left(\widehat{\beta}_{4}\right)$ & 0.065 & 0.090 & 0.140 \\
\hline
\end{tabular}




\section{Table 9}

Simulation results for the cluster-weighted AFT model when the cluster size is only related to the cluster effect (Beta $1=0.8$ Beta $2=1$, Beta3 $=-0.75$, Beta $4=-1.5$, alpha $=0.25$,

$$
\mathrm{r} 1=1.5, \mathrm{r} 2=3, \mathrm{z} 3=\mathrm{N}(1,0.15) \text {, error }=0.05) \text {. }
$$

\begin{tabular}{|c|c|c|c|c|}
\hline \multirow[t]{2}{*}{ Number of cluster } & & \multicolumn{3}{|c|}{ Censoring rate } \\
\hline & & 0.25 & 0.5 & 0.75 \\
\hline \multirow[t]{12}{*}{30} & $\operatorname{Bias}\left(\widehat{\beta}_{1}\right)$ & 0.018 & 0.031 & 0.009 \\
\hline & $\operatorname{e.sd}\left(\widehat{\beta}_{1}\right)$ & 0.297 & 0.338 & 0.493 \\
\hline & $\operatorname{sd}\left(\widehat{\beta}_{1}\right)$ & 0.256 & 0.285 & 0.421 \\
\hline & $\operatorname{Bias}\left(\widehat{\beta}_{2}\right)$ & -0.008 & -0.057 & -0.053 \\
\hline & $\operatorname{e.sd}\left(\widehat{\beta}_{2}\right)$ & 0.414 & 0.485 & 0.667 \\
\hline & $\operatorname{sd}\left(\widehat{\beta}_{2}\right)$ & 0.361 & 0.405 & 0.561 \\
\hline & $\operatorname{Bias}\left(\widehat{\beta}_{3}\right)$ & 0.003 & -0.007 & 0.007 \\
\hline & $\operatorname{e.sd}\left(\widehat{\beta}_{3}\right)$ & 0.282 & 0.322 & 0.478 \\
\hline & $\operatorname{sd}\left(\widehat{\beta}_{3}\right)$ & 0.244 & 0.273 & 0.403 \\
\hline & $\operatorname{Bias}\left(\widehat{\beta}_{4}\right)$ & 0.002 & 0.043 & 0.046 \\
\hline & $\operatorname{e.sd}\left(\widehat{\beta}_{4}\right)$ & 0.396 & 0.463 & 0.632 \\
\hline & $\operatorname{sd}\left(\widehat{\beta}_{4}\right)$ & 0.343 & 0.385 & 0.532 \\
\hline \multirow[t]{12}{*}{200} & $\operatorname{Bias}\left(\widehat{\beta}_{1}\right)$ & 0.017 & 0.028 & 0.018 \\
\hline & $\operatorname{e.sd}\left(\widehat{\beta}_{1}\right)$ & 0.124 & 0.141 & 0.196 \\
\hline & $\operatorname{sd}\left(\widehat{\beta}_{1}\right)$ & 0.113 & 0.131 & 0.188 \\
\hline & $\operatorname{Bias}\left(\widehat{\beta}_{2}\right)$ & -0.019 & -0.064 & -0.062 \\
\hline & e.sd $\left(\widehat{\beta}_{2}\right)$ & 0.166 & 0.192 & 0.278 \\
\hline & $\operatorname{sd}\left(\widehat{\beta}_{2}\right)$ & 0.158 & 0.183 & 0.254 \\
\hline & $\operatorname{Bias}\left(\widehat{\beta}_{3}\right)$ & 0.001 & -0.006 & 0.004 \\
\hline & e.sd $\left(\widehat{\beta}_{3}\right)$ & 0.116 & 0.132 & 0.186 \\
\hline & $\operatorname{sd}\left(\widehat{\beta}_{3}\right)$ & 0.107 & 0.124 & 0.180 \\
\hline & $\operatorname{Bias}\left(\widehat{\beta}_{4}\right)$ & 0.012 & 0.045 & 0.039 \\
\hline & $\operatorname{e.sd}\left(\widehat{\beta}_{4}\right)$ & 0.156 & 0.179 & 0.260 \\
\hline & $\operatorname{sd}\left(\widehat{\beta}_{4}\right)$ & 0.150 & 0.173 & 0.239 \\
\hline
\end{tabular}




\section{Table 10}

Simulation results for the non-cluster-weighted AFT model when the cluster size is only related to the cluster effect $($ Beta $1=0.8$ Beta $2=1$, Beta $3=-0.75$, Beta4 $=-1.5$, alpha $=0.25$, $\mathrm{rl}=1.5, \mathrm{r} 2=3, \mathrm{z} 3=\mathrm{N}(1,0.15)$, error $=0.05)$.

\begin{tabular}{|c|c|c|c|c|}
\hline \multirow[t]{2}{*}{ Number of cluster } & & \multicolumn{3}{|c|}{ Censoring rate } \\
\hline & & 0.25 & 0.5 & 0.75 \\
\hline \multirow[t]{12}{*}{30} & $\operatorname{Bias}\left(\widehat{\beta}_{1}\right)$ & 0.150 & 0.147 & 0.113 \\
\hline & $\operatorname{e} . \operatorname{sd}\left(\widehat{\beta}_{1}\right)$ & 0.241 & 0.280 & 0.472 \\
\hline & $\operatorname{sd}\left(\widehat{\beta}_{1}\right)$ & 0.206 & 0.252 & 0.399 \\
\hline & $\operatorname{Bias}\left(\widehat{\beta}_{2}\right)$ & 0.002 & -0.043 & -0.067 \\
\hline & $\operatorname{e.sd}\left(\widehat{\beta}_{2}\right)$ & 0.331 & 0.398 & 0.606 \\
\hline & $\operatorname{sd}\left(\widehat{\beta}_{2}\right)$ & 0.286 & 0.346 & 0.523 \\
\hline & $\operatorname{Bias}\left(\widehat{\beta}_{3}\right)$ & 0.010 & 0.012 & 0.025 \\
\hline & $\operatorname{e} . \operatorname{sd}\left(\widehat{\beta}_{3}\right)$ & 0.216 & 0.259 & 0.457 \\
\hline & $\operatorname{sd}\left(\widehat{\beta}_{3}\right)$ & 0.199 & 0.243 & 0.383 \\
\hline & $\operatorname{Bias}\left(\widehat{\beta}_{4}\right)$ & -0.006 & 0.033 & 0.068 \\
\hline & $\operatorname{e.sd}\left(\widehat{\beta}_{4}\right)$ & 0.295 & 0.363 & 0.569 \\
\hline & $\operatorname{sd}\left(\widehat{\beta}_{4}\right)$ & 0.276 & 0.329 & 0.494 \\
\hline \multirow[t]{12}{*}{200} & $\operatorname{Bias}\left(\widehat{\beta}_{1}\right)$ & 0.183 & 0.184 & 0.147 \\
\hline & $\operatorname{e.sd}\left(\widehat{\beta}_{1}\right)$ & 0.125 & 0.148 & 0.219 \\
\hline & $\operatorname{sd}\left(\widehat{\beta}_{1}\right)$ & 0.096 & 0.123 & 0.206 \\
\hline & $\operatorname{Bias}\left(\widehat{\beta}_{2}\right)$ & -0.014 & -0.055 & -0.047 \\
\hline & $\operatorname{e} . \operatorname{sd}\left(\widehat{\beta}_{2}\right)$ & 0.161 & 0.191 & 0.309 \\
\hline & $\operatorname{sd}\left(\widehat{\beta}_{2}\right)$ & 0.131 & 0.169 & 0.269 \\
\hline & $\operatorname{Bias}\left(\widehat{\beta}_{3}\right)$ & -0.001 & 0.003 & 0.032 \\
\hline & $\operatorname{e} . \operatorname{sd}\left(\widehat{\beta}_{3}\right)$ & 0.113 & 0.138 & 0.213 \\
\hline & $\operatorname{sd}\left(\widehat{\beta}_{3}\right)$ & 0.092 & 0.118 & 0.197 \\
\hline & $\operatorname{Bias}\left(\widehat{\beta}_{4}\right)$ & 0.003 & 0.032 & 0.025 \\
\hline & $\operatorname{e} . \operatorname{sd}\left(\widehat{\beta}_{4}\right)$ & 0.141 & 0.174 & 0.287 \\
\hline & $\operatorname{sd}\left(\widehat{\beta}_{4}\right)$ & 0.126 & 0.160 & 0.252 \\
\hline
\end{tabular}


Table 11

Simulation results for the cluster-weighted AFT model when the cluster size is only related to the cluster effect $($ Beta $1=0.3$ Beta $2=0.4$, Beta $3=-0.2$, Beta $4=-0.3$, alpha $=0.25$,

$$
\mathrm{r} 1=1.5, \mathrm{r} 2=3, \mathrm{z} 3=\mathrm{N}(1,0.15), \text { error }=0.2) .
$$

\begin{tabular}{|c|c|c|c|c|}
\hline \multirow[t]{2}{*}{ Number of cluster } & & \multicolumn{3}{|c|}{ Censoring rate } \\
\hline & & 0.25 & 0.5 & 0.75 \\
\hline \multirow[t]{12}{*}{30} & $\operatorname{Bias}\left(\widehat{\beta}_{1}\right)$ & 0.024 & 0.050 & 0.052 \\
\hline & $\operatorname{e.sd}\left(\widehat{\beta}_{1}\right)$ & 0.319 & 0.369 & 0.494 \\
\hline & $\operatorname{sd}\left(\widehat{\beta}_{1}\right)$ & 0.219 & 0.323 & 0.412 \\
\hline & $\operatorname{Bias}\left(\widehat{\beta}_{2}\right)$ & -0.067 & -0.105 & -0.132 \\
\hline & $\operatorname{e.sd}\left(\widehat{\beta}_{2}\right)$ & 0.499 & 0.626 & 0.919 \\
\hline & $\operatorname{sd}\left(\widehat{\beta}_{2}\right)$ & 0.443 & 0.521 & 0.703 \\
\hline & $\operatorname{Bias}\left(\widehat{\beta}_{3}\right)$ & 0.010 & -0.001 & 0.001 \\
\hline & $\operatorname{e.sd}\left(\widehat{\beta}_{3}\right)$ & 0.305 & 0.355 & 0.482 \\
\hline & $\operatorname{sd}\left(\widehat{\beta}_{3}\right)$ & 0.281 & 0.313 & 0.402 \\
\hline & $\operatorname{Bias}\left(\widehat{\beta}_{4}\right)$ & 0.045 & 0.064 & 0.074 \\
\hline & $\operatorname{e.sd}\left(\widehat{\beta}_{4}\right)$ & 0.481 & 0.606 & 0.886 \\
\hline & $\operatorname{sd}\left(\widehat{\beta}_{4}\right)$ & 0.427 & 0.504 & 0.682 \\
\hline \multirow[t]{12}{*}{200} & $\operatorname{Bias}\left(\widehat{\beta}_{1}\right)$ & 0.022 & 0.058 & 0.075 \\
\hline & $\operatorname{e.sd}\left(\widehat{\beta}_{1}\right)$ & 0.121 & 0.152 & 0.216 \\
\hline & $\operatorname{sd}\left(\widehat{\beta}_{1}\right)$ & 0.122 & 0.143 & 0.202 \\
\hline & $\operatorname{Bias}\left(\widehat{\beta}_{2}\right)$ & -0.062 & -0.093 & -0.108 \\
\hline & $\operatorname{e.sd}\left(\widehat{\beta}_{2}\right)$ & 0.189 & 0.247 & 0.364 \\
\hline & $\operatorname{sd}\left(\widehat{\beta}_{2}\right)$ & 0.186 & 0.233 & 0.338 \\
\hline & $\operatorname{Bias}\left(\widehat{\beta}_{3}\right)$ & 0.011 & -0.002 & -0.006 \\
\hline & $\operatorname{e.sd}\left(\widehat{\beta}_{3}\right)$ & 0.116 & 0.145 & 0.208 \\
\hline & $\operatorname{sd}\left(\widehat{\beta}_{3}\right)$ & 0.117 & 0.138 & 0.197 \\
\hline & $\operatorname{Bias}\left(\widehat{\beta}_{4}\right)$ & 0.036 & 0.045 & 0.046 \\
\hline & $\operatorname{e.sd}\left(\widehat{\beta}_{4}\right)$ & 0.183 & 0.239 & 0.353 \\
\hline & $\operatorname{sd}\left(\widehat{\beta}_{4}\right)$ & 0.179 & 0.225 & 0.327 \\
\hline
\end{tabular}


Table 12

Simulation results for the non-cluster-weighted AFT model when the cluster size is only related to the cluster effect (Beta $1=0.3$ Beta $2=0.4$, Beta $3=-0.2$, Beta $4=-0.3$, alpha $=0.25$,

$$
\mathrm{r} 1=1.5, \mathrm{r} 2=3, \mathrm{z} 3=\mathrm{N}(1,0.15) \text {, error }=0.2) \text {. }
$$

\begin{tabular}{llrrr}
\hline Number of cluster & & \multicolumn{3}{c}{ Censoring rate } \\
& & 0.25 & 0.5 & 0.75 \\
\hline 30 & $\operatorname{Bias}\left(\widehat{\beta}_{1}\right)$ & 0.167 & 0.186 & 0.169 \\
& $\operatorname{e.sd}\left(\widehat{\beta}_{1}\right)$ & 0.261 & 0.317 & 0.461 \\
& $\operatorname{sd}\left(\widehat{\beta}_{1}\right)$ & 0.236 & 0.279 & 0.376 \\
& $\operatorname{Bias}\left(\widehat{\beta}_{2}\right)$ & -0.070 & -0.121 & -0.144 \\
& $\operatorname{e.sd}\left(\widehat{\beta}_{2}\right)$ & 0.405 & 0.560 & 0.858 \\
& $\operatorname{sd}\left(\widehat{\beta}_{2}\right)$ & 0.369 & 0.467 & 0.646 \\
& $\operatorname{Bias}\left(\widehat{\beta}_{3}\right)$ & 0.010 & 0.003 & 0.014 \\
& $\operatorname{e.sd}\left(\widehat{\beta}_{3}\right)$ & 0.238 & 0.299 & 0.449 \\
& $\operatorname{sd}\left(\widehat{\beta}_{3}\right)$ & 0.232 & 0.275 & 0.369 \\
& $\operatorname{Bias}\left(\widehat{\beta}_{4}\right)$ & 0.045 & 0.075 & 0.075 \\
& $\operatorname{e} . \operatorname{sd}\left(\widehat{\beta}_{4}\right)$ & 0.377 & 0.537 & 0.827 \\
& $\operatorname{sd}\left(\widehat{\beta}_{4}\right)$ & 0.359 & 0.454 & 0.627 \\
& & & \\
& $\operatorname{Bias}\left(\widehat{\beta}_{1}\right)$ & 0.187 & 0.232 & 0.235 \\
& $\operatorname{e.sd}\left(\widehat{\beta}_{1}\right)$ & 0.113 & 0.165 & 0.249 \\
& $\operatorname{sd}\left(\widehat{\beta}_{1}\right)$ & 0.102 & 0.144 & 0.219 \\
& $\operatorname{Bias}\left(\widehat{\beta}_{2}\right)$ & -0.075 & -0.121 & -0.136 \\
& $\operatorname{e.sd}\left(\widehat{\beta}_{2}\right)$ & 0.175 & 0.268 & 0.424 \\
& $\operatorname{sd}\left(\widehat{\beta}_{2}\right)$ & 0.159 & 0.237 & 0.367 \\
& $\operatorname{Bias}\left(\widehat{\beta}_{3}\right)$ & 0.014 & -0.001 & 0.005 \\
& $\operatorname{e.sd}\left(\widehat{\beta}_{3}\right)$ & 0.101 & 0.150 & 0.237 \\
& $\operatorname{sd}\left(\widehat{\beta}_{3}\right)$ & 0.100 & 0.141 & 0.215 \\
& $\operatorname{Bias}\left(\widehat{\beta}_{4}\right)$ & 0.045 & 0.063 & 0.061 \\
& $\operatorname{e.sd}\left(\widehat{\beta}_{4}\right)$ & 0.163 & 0.257 & 0.407 \\
$\operatorname{sd}\left(\widehat{\beta}_{4}\right)$ & 0.156 & 0.230 & 0.356 \\
\hline & & &
\end{tabular}




\section{Table 13}

Simulation results for the cluster-weighted AFT model when the cluster size is related to both the cluster effect and the binary covariate ((Beta1 $=0.3$ Beta $2=0.4$, Beta $3=-0.2$,

$$
\text { Beta4 }=-0.3 \text {, alpha }=0.25, \mathrm{r} 1=1.5, \mathrm{r} 2=4, \mathrm{r} 3=-4, \mathrm{z} 3=\mathrm{N}(1,0.3) \text {,error }=0.05) \text {. }
$$

\begin{tabular}{llrrr}
\hline Number of cluster & & \multicolumn{3}{c}{ Censoring rate } \\
& & 0.25 & 0.5 & \multicolumn{1}{c}{0.75} \\
\hline 30 & $\operatorname{Bias}\left(\widehat{\beta}_{1}\right)$ & -0.009 & -0.010 & -0.007 \\
& $\operatorname{e.sd}\left(\widehat{\beta}_{1}\right)$ & 0.155 & 0.174 & 0.216 \\
& $\operatorname{sd}\left(\widehat{\beta}_{1}\right)$ & 0.139 & 0.151 & 0.180 \\
& $\operatorname{Bias}\left(\widehat{\beta}_{2}\right)$ & -0.003 & -0.011 & -0.033 \\
& $\operatorname{e.sd}\left(\widehat{\beta}_{2}\right)$ & 0.382 & 0.482 & 1.032 \\
& $\operatorname{sd}\left(\widehat{\beta}_{2}\right)$ & 0.313 & 0.375 & 0.548 \\
& $\operatorname{Bias}\left(\widehat{\beta}_{3}\right)$ & 0.021 & 0.023 & 0.020 \\
& $\operatorname{e.sd}\left(\widehat{\beta}_{3}\right)$ & 0.135 & 0.153 & 0.193 \\
& $\operatorname{sd}\left(\widehat{\beta}_{3}\right)$ & 0.119 & 0.131 & 0.159 \\
& $\operatorname{Bias}\left(\widehat{\beta}_{4}\right)$ & -0.011 & -0.011 & -0.019 \\
& $\operatorname{e.sd}\left(\widehat{\beta}_{4}\right)$ & 0.345 & 0.441 & 0.913 \\
& $\operatorname{sd}\left(\widehat{\beta}_{4}\right)$ & 0.28 & 0.336 & 0.493 \\
& & & \\
& $\operatorname{Bias}\left(\widehat{\beta}_{1}\right)$ & -0.002 & 0.001 & 0.003 \\
& $\operatorname{e.sd}\left(\widehat{\beta}_{1}\right)$ & 0.062 & 0.069 & 0.084 \\
& $\operatorname{sd}\left(\widehat{\beta}_{1}\right)$ & 0.059 & 0.066 & 0.08 \\
& $\operatorname{Bias}\left(\widehat{\beta}_{2}\right)$ & -0.031 & -0.042 & -0.068 \\
& $\operatorname{e.sd}\left(\widehat{\beta}_{2}\right)$ & 0.134 & 0.159 & 0.231 \\
& $\operatorname{sd}\left(\widehat{\beta}_{2}\right)$ & 0.128 & 0.15 & 0.206 \\
& $\operatorname{Bias}\left(\widehat{\beta}_{3}\right)$ & 0.011 & 0.008 & 0.007 \\
& $\operatorname{e.sd}\left(\widehat{\beta}_{3}\right)$ & 0.055 & 0.061 & 0.075 \\
& $\operatorname{sd}\left(\widehat{\beta}_{3}\right)$ & 0.052 & 0.058 & 0.072 \\
$\operatorname{Bias}\left(\widehat{\beta}_{4}\right)$ & 0.021 & 0.028 & 0.038 \\
& $\operatorname{e.sd}\left(\widehat{\beta}_{4}\right)$ & 0.119 & 0.143 & 0.204 \\
$\operatorname{sd}\left(\widehat{\beta}_{4}\right)$ & 0.115 & 0.134 & 0.182 \\
\hline & & & &
\end{tabular}


Table 14

Simulation results for the non-cluster-weighted AFT model when the cluster size is related to both the cluster effect and the binary covariate $($ Beta1 $=0.3$ Beta2 $=0.4$, Beta3 $=-$ 0.2 , Beta4 $=-0.3$, alpha $=0.25, \mathrm{r} 1=1.5, \mathrm{r} 2=4, \mathrm{r} 3=-4, \mathrm{z} 3=\mathrm{N}(1,0.3)$,error $=0.05)$.

\begin{tabular}{llrrr}
\hline Number of cluster & & \multicolumn{3}{c}{ Censoring rate } \\
& & 0.25 & 0.5 & 0.75 \\
\hline 30 & $\operatorname{Bias}\left(\widehat{\beta}_{1}\right)$ & 0.181 & 0.178 & 0.178 \\
& $\operatorname{e.sd}\left(\widehat{\beta}_{1}\right)$ & 0.144 & 0.157 & 0.189 \\
& $\operatorname{sd}\left(\widehat{\beta}_{1}\right)$ & 0.086 & 0.102 & 0.146 \\
& $\operatorname{Bias}\left(\widehat{\beta}_{2}\right)$ & -0.173 & -0.181 & -0.209 \\
& $\operatorname{e} \operatorname{sd}\left(\widehat{\beta}_{2}\right)$ & 0.383 & 0.484 & 1.035 \\
& $\operatorname{sd}\left(\widehat{\beta}_{2}\right)$ & 0.294 & 0.361 & 0.504 \\
& $\operatorname{Bias}\left(\widehat{\beta}_{3}\right)$ & 0.014 & 0.017 & 0.013 \\
& $\operatorname{e} \operatorname{sd}\left(\widehat{\beta}_{3}\right)$ & 0.089 & 0.105 & 0.148 \\
& $\operatorname{sd}\left(\widehat{\beta}_{3}\right)$ & 0.079 & 0.094 & 0.133 \\
& $\operatorname{Bias}\left(\widehat{\beta}_{4}\right)$ & -0.001 & -0.001 & -0.009 \\
& $\operatorname{e.sd}\left(\widehat{\beta}_{4}\right)$ & 0.338 & 0.434 & 0.909 \\
& $\operatorname{sd}\left(\widehat{\beta}_{4}\right)$ & 0.267 & 0.326 & 0.455 \\
& & & \\
& $\operatorname{Bias}\left(\widehat{\beta}_{1}\right)$ & 0.216 & 0.217 & 0.213 \\
& $\operatorname{e.sd}\left(\widehat{\beta}_{1}\right)$ & 0.069 & 0.07 & 0.085 \\
& $\operatorname{sd}\left(\widehat{\beta}_{1}\right)$ & 0.039 & 0.049 & 0.075 \\
& $\operatorname{Bias}\left(\widehat{\beta}_{2}\right)$ & -0.225 & -0.237 & -0.257 \\
& $\operatorname{e.sd}\left(\widehat{\beta}_{2}\right)$ & 0.145 & 0.173 & 0.248 \\
& $\operatorname{sd}\left(\widehat{\beta}_{2}\right)$ & 0.127 & 0.153 & 0.213 \\
$\operatorname{Bias}\left(\widehat{\beta}_{3}\right)$ & 0.009 & 0.009 & 0.012 \\
& $\operatorname{e.sd}\left(\widehat{\beta}_{3}\right)$ & 0.043 & 0.048 & 0.069 \\
& $\operatorname{sd}\left(\widehat{\beta}_{3}\right)$ & 0.036 & 0.045 & 0.068 \\
& $\operatorname{Bias}\left(\widehat{\beta}_{4}\right)$ & 0.025 & 0.032 & 0.037 \\
& $\operatorname{e} \operatorname{sd}\left(\widehat{\beta}_{4}\right)$ & 0.119 & 0.148 & 0.215 \\
$\operatorname{sd}\left(\widehat{\beta}_{4}\right)$ & 0.114 & 0.137 & 0.188 \\
\hline & & &
\end{tabular}


Table 15

Simulation results for the cluster-weighted AFT model when the cluster size is related to both the cluster effect and the binary covariate (Beta1 $=0.3$ Beta2 $=0.4$, Beta $3=-0.2$,

Beta4 $=-0.3$, alpha $=0.25, \mathrm{r} 1=1.5, \mathrm{r} 2=4, \mathrm{r} 3=-4, \mathrm{z} 3=\mathrm{N}(1,0.15)$, error $=0.05)$.

\begin{tabular}{|c|c|c|c|c|}
\hline \multirow[t]{2}{*}{ Number of cluster } & & \multicolumn{3}{|c|}{ Censoring rate } \\
\hline & & 0.25 & 0.5 & 0.75 \\
\hline \multirow[t]{12}{*}{30} & $\operatorname{Bias}\left(\widehat{\beta}_{1}\right)$ & -0.026 & -0.035 & -0.023 \\
\hline & e.sd $\left(\widehat{\beta}_{1}\right)$ & 0.278 & 0.311 & 0.400 \\
\hline & $\operatorname{sd}\left(\widehat{\beta}_{1}\right)$ & 0.249 & 0.272 & 0.328 \\
\hline & $\operatorname{Bias}\left(\widehat{\beta}_{2}\right)$ & 0.045 & 0.027 & -0.049 \\
\hline & $\operatorname{e.sd}\left(\widehat{\beta}_{2}\right)$ & 0.689 & 0.839 & 1.414 \\
\hline & $\operatorname{sd}\left(\widehat{\beta}_{2}\right)$ & 0.579 & 0.672 & 0.843 \\
\hline & $\operatorname{Bias}\left(\widehat{\beta}_{3}\right)$ & 0.034 & 0.043 & 0.033 \\
\hline & $\operatorname{e.sd}\left(\widehat{\beta}_{3}\right)$ & 0.269 & 0.299 & 0.388 \\
\hline & $\operatorname{sd}\left(\widehat{\beta}_{3}\right)$ & 0.238 & 0.261 & 0.317 \\
\hline & $\operatorname{Bias}\left(\widehat{\beta}_{4}\right)$ & -0.057 & -0.049 & -0.008 \\
\hline & $\operatorname{e.sd}\left(\widehat{\beta}_{4}\right)$ & 0.669 & 0.819 & 1.379 \\
\hline & $\operatorname{sd}\left(\widehat{\beta}_{4}\right)$ & 0.559 & 0.651 & 0.814 \\
\hline \multirow[t]{12}{*}{200} & $\operatorname{Bias}\left(\widehat{\beta}_{1}\right)$ & -0.007 & -0.006 & 0.003 \\
\hline & e.sd $\left(\widehat{\beta}_{1}\right)$ & 0.112 & 0.124 & 0.152 \\
\hline & $\operatorname{sd}\left(\widehat{\beta}_{1}\right)$ & 0.107 & 0.118 & 0.146 \\
\hline & $\operatorname{Bias}\left(\widehat{\beta}_{2}\right)$ & -0.038 & -0.051 & -0.079 \\
\hline & $\operatorname{e.sd}\left(\widehat{\beta}_{2}\right)$ & 0.237 & 0.286 & 0.408 \\
\hline & $\operatorname{sd}\left(\widehat{\beta}_{2}\right)$ & 0.229 & 0.266 & 0.368 \\
\hline & $\operatorname{Bias}\left(\widehat{\beta}_{3}\right)$ & 0.011 & 0.009 & 0.003 \\
\hline & e.sd $\left(\widehat{\beta}_{3}\right)$ & 0.108 & 0.120 & 0.148 \\
\hline & $\operatorname{sd}\left(\widehat{\beta}_{3}\right)$ & 0.103 & 0.115 & 0.142 \\
\hline & $\operatorname{Bias}\left(\widehat{\beta}_{4}\right)$ & 0.029 & 0.038 & 0.051 \\
\hline & e.sd $\left(\widehat{\beta}_{4}\right)$ & 0.231 & 0.279 & 0.394 \\
\hline & $\operatorname{sd}\left(\widehat{\beta}_{4}\right)$ & 0.222 & 0.258 & 0.356 \\
\hline
\end{tabular}




\section{Table 16}

Simulation results for the non-cluster-weighted AFT model when the cluster size is related to both the cluster effect and the binary covariate (Beta1 $=0.3$ Beta2 $=0.4$, Beta3 $=-$

0.2 , Beta4 $=-0.3$, alpha $=0.25, \mathrm{rl}=1.5, \mathrm{r} 2=4, \mathrm{r} 3=-4, \mathrm{z} 3=\mathrm{N}(1,0.15)$, error $=0.05)$.

\begin{tabular}{|c|c|c|c|c|}
\hline \multirow[t]{2}{*}{ Number of cluster } & & \multicolumn{3}{|c|}{ Censoring rate } \\
\hline & & 0.25 & 0.5 & 0.75 \\
\hline \multirow[t]{12}{*}{30} & $\operatorname{Bias}\left(\widehat{\beta}_{1}\right)$ & 0.172 & 0.164 & 0.172 \\
\hline & $\operatorname{e.sd}\left(\widehat{\beta}_{1}\right)$ & 0.201 & 0.234 & 0.319 \\
\hline & $\operatorname{sd}\left(\widehat{\beta}_{1}\right)$ & 0.161 & 0.192 & 0.274 \\
\hline & $\operatorname{Bias}\left(\widehat{\beta}_{2}\right)$ & -0.126 & -0.156 & -0.232 \\
\hline & $\operatorname{e.sd}\left(\widehat{\beta}_{2}\right)$ & 0.676 & 0.828 & 1.396 \\
\hline & $\operatorname{sd}\left(\widehat{\beta}_{2}\right)$ & 0.552 & 0.660 & 0.878 \\
\hline & $\operatorname{Bias}\left(\widehat{\beta}_{3}\right)$ & 0.019 & 0.027 & 0.016 \\
\hline & $\operatorname{e.sd}\left(\widehat{\beta}_{3}\right)$ & 0.168 & 0.205 & 0.297 \\
\hline & $\operatorname{sd}\left(\widehat{\beta}_{3}\right)$ & 0.158 & 0.188 & 0.267 \\
\hline & $\operatorname{Bias}\left(\widehat{\beta}_{4}\right)$ & -0.046 & -0.026 & 0.008 \\
\hline & $\operatorname{e.sd}\left(\widehat{\beta}_{4}\right)$ & 0.653 & 0.808 & 1.362 \\
\hline & $\operatorname{sd}\left(\widehat{\beta}_{4}\right)$ & 0.535 & 0.642 & 0.854 \\
\hline \multirow[t]{12}{*}{200} & $\operatorname{Bias}\left(\widehat{\beta}_{1}\right)$ & 0.208 & 0.208 & 0.209 \\
\hline & $\operatorname{e.sd}\left(\widehat{\beta}_{1}\right)$ & 0.087 & 0.102 & 0.149 \\
\hline & $\operatorname{sd}\left(\widehat{\beta}_{1}\right)$ & 0.072 & 0.089 & 0.135 \\
\hline & $\operatorname{Bias}\left(\widehat{\beta}_{2}\right)$ & -0.231 & -0.242 & -0.263 \\
\hline & $\operatorname{e.sd}\left(\widehat{\beta}_{2}\right)$ & 0.238 & 0.296 & 0.432 \\
\hline & $\operatorname{sd}\left(\widehat{\beta}_{2}\right)$ & 0.226 & 0.270 & 0.380 \\
\hline & $\operatorname{Bias}\left(\widehat{\beta}_{3}\right)$ & 0.012 & 0.011 & 0.010 \\
\hline & $\operatorname{e.sd}\left(\widehat{\beta}_{3}\right)$ & 0.072 & 0.089 & 0.141 \\
\hline & $\operatorname{sd}\left(\widehat{\beta}_{3}\right)$ & 0.070 & 0.087 & 0.132 \\
\hline & $\operatorname{Bias}\left(\widehat{\beta}_{4}\right)$ & 0.034 & 0.041 & 0.047 \\
\hline & $\operatorname{e.sd}\left(\widehat{\beta}_{4}\right)$ & 0.227 & 0.284 & 0.415 \\
\hline & $\operatorname{sd}\left(\widehat{\beta}_{4}\right)$ & 0.221 & 0.262 & 0.366 \\
\hline
\end{tabular}


In addition, we show the Probability-Probability plots for the estimated regression coefficients. We generated the nominal versus the actual coverage rate by varying the significance level in the range $(0.02,0.98)$. In Figure 1 , the cluster size was only related to the cluster effect $\alpha_{i}$. The parameters used are the same as those in Table 5 and Table 6 with censoring rate equal to 25 percent and the number of cluster equal to 200 . The empirical coverage rates for the cluster-weighted AFT model are close to the nominal level, while the empirical coverage rates of $\beta_{1}$ for the non-cluster-weighted AFT model are substantially below the nominal levels, indicating defective for the non-clusterweighted AFT model in the situation. In Figure 2, 3 and 4, the cluster size was only related to the cluster effect $\alpha_{i}$. The parameters used are the same as those in Table 7-8, Table 9-10, and table 11-12 respectively with censoring rate equal to 25 percentage and the number of cluster equal to 200 . We find similar pattern in those figures with that in Figure 1. 

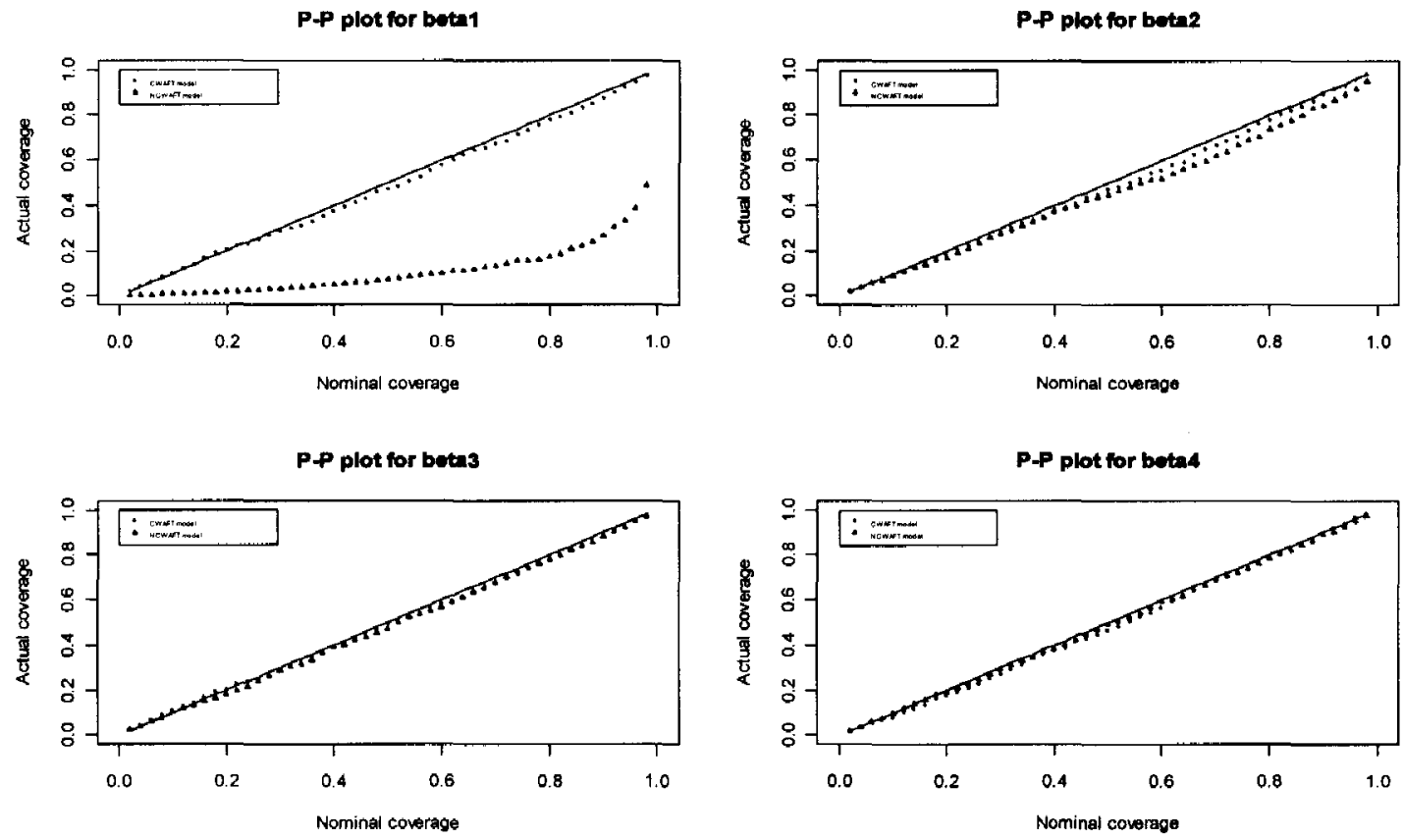

Figure 1. Probability-Probability plots for parameters for two competing methods when the cluster size is only related to the cluster effect using the same parameters in Table 5-6.
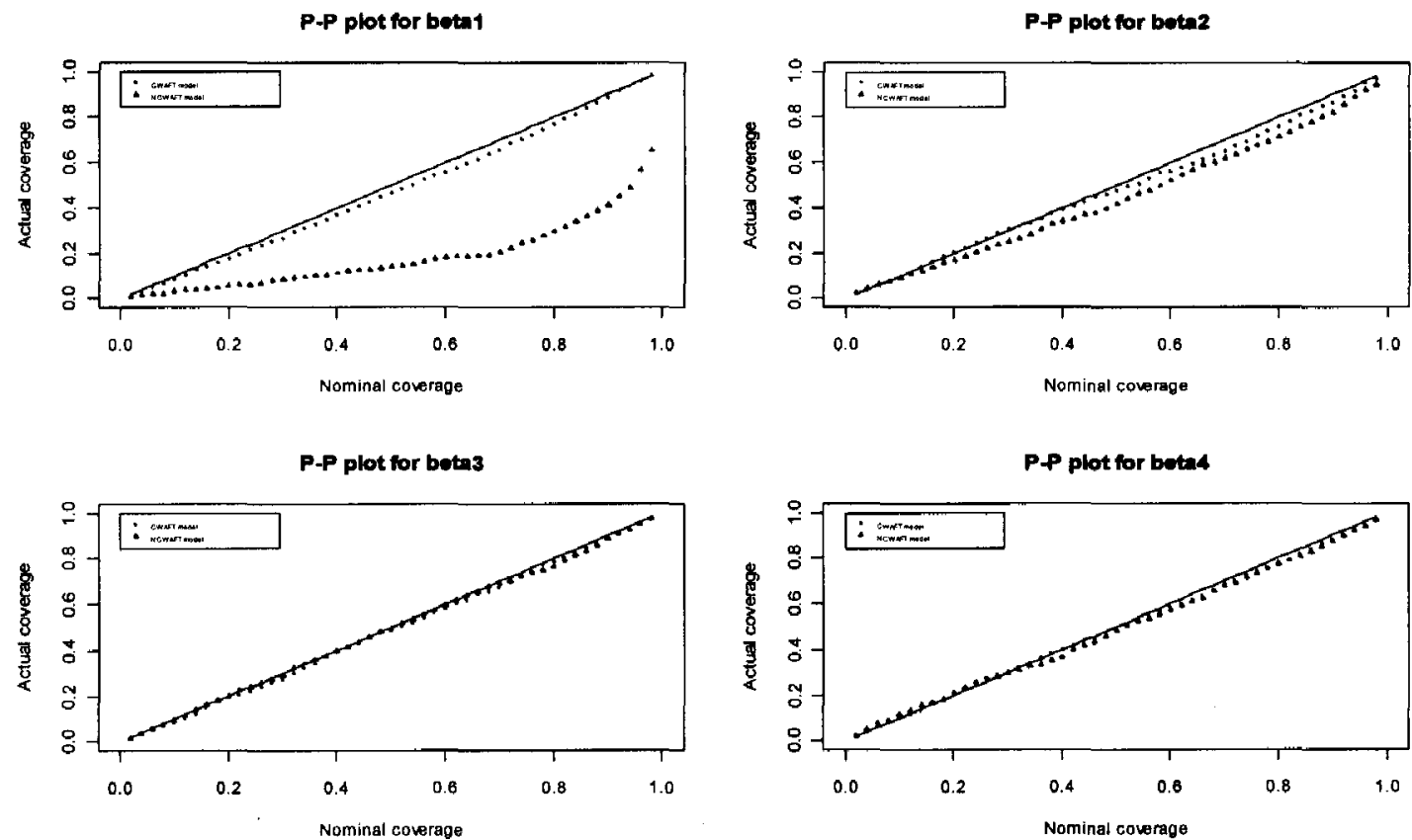

Figure 2. Probability-Probability plots for parameters for two competing methods when the cluster size is only related to the cluster effect using the same parameters in Table 7-8. 

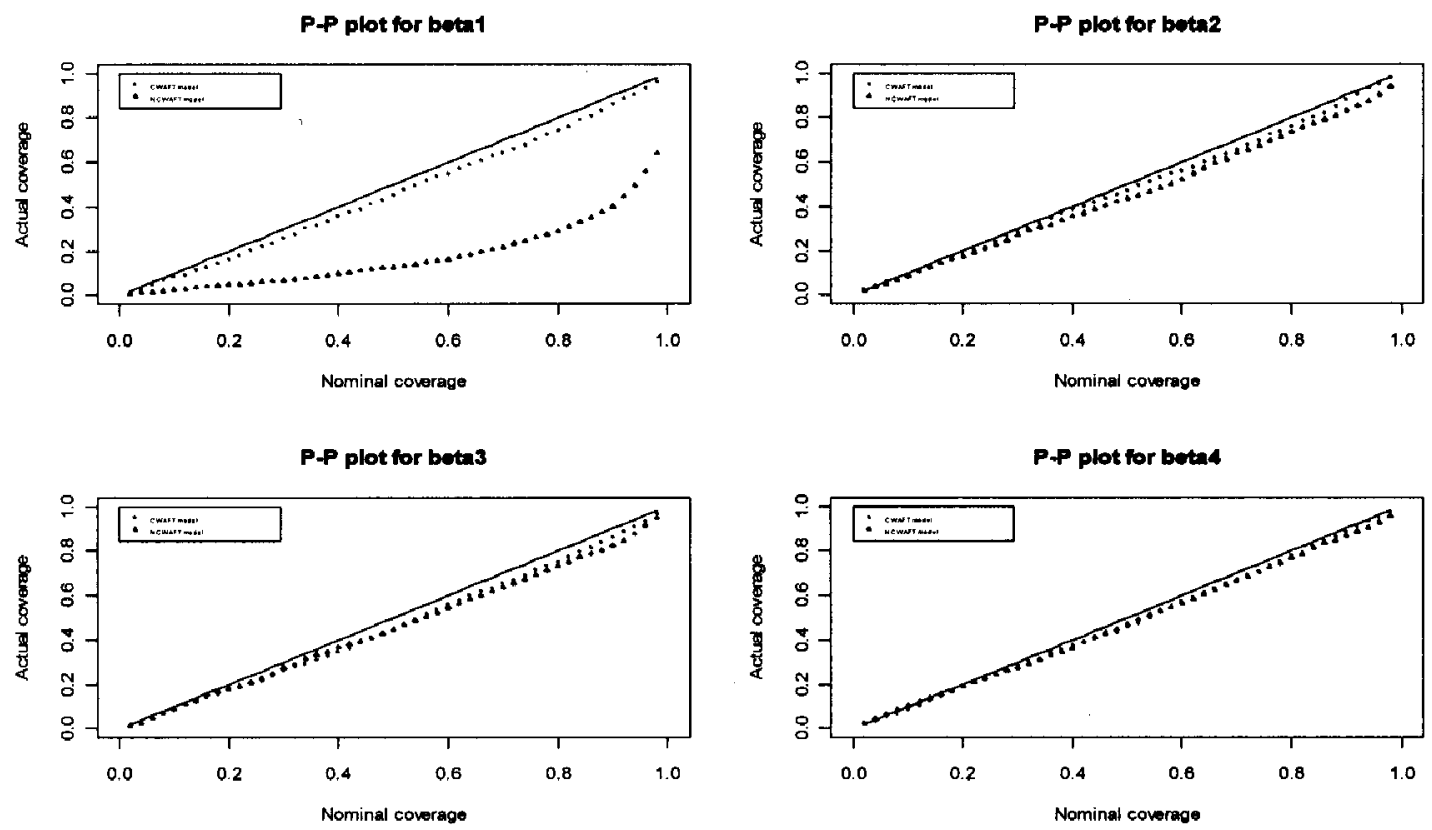

Figure 3. Probability-Probability plots for parameters for two competing methods when the cluster size is only related to the cluster effect using the same parameters in Table 9-10.
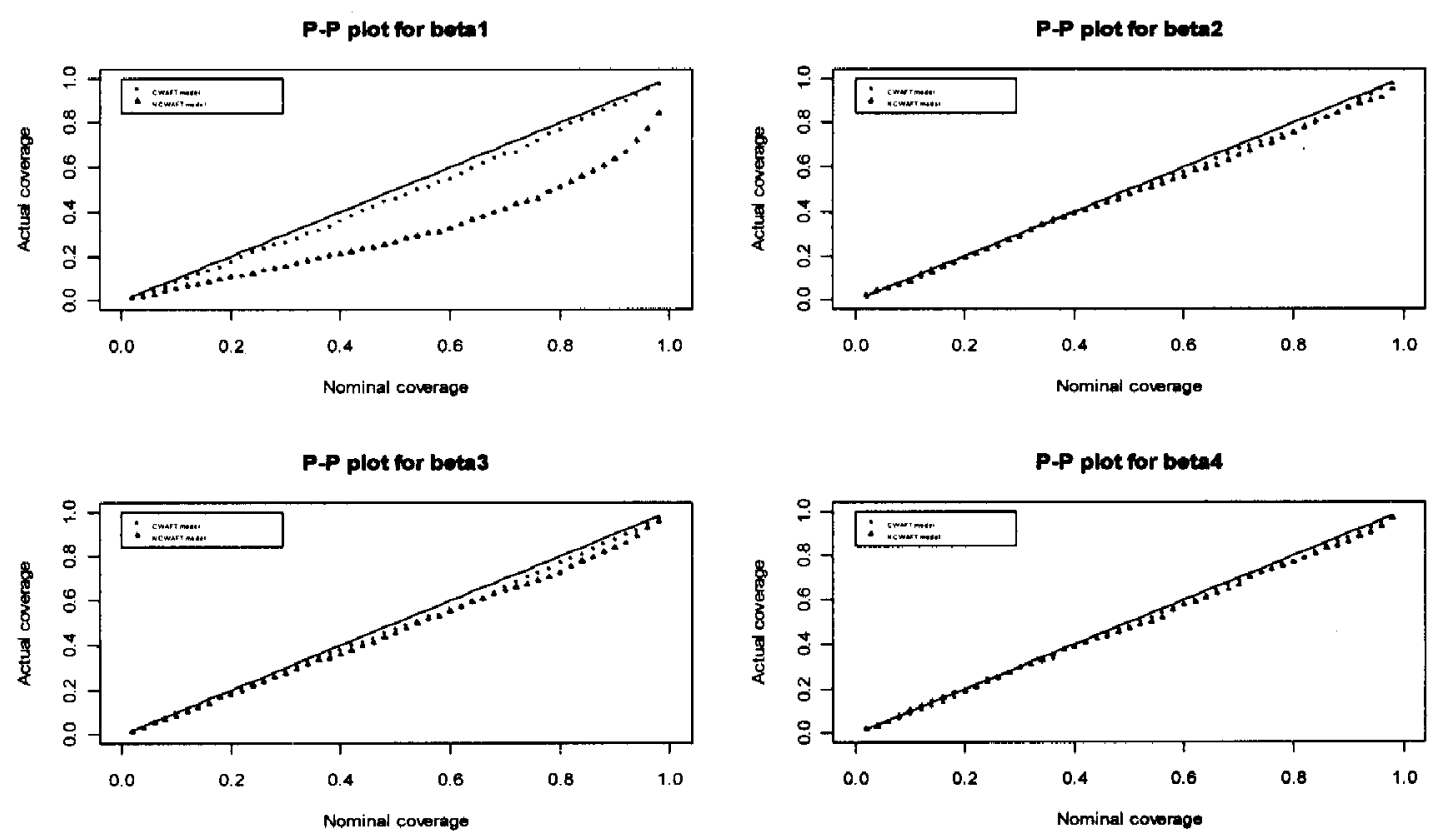

Figure 4. Probability-Probability plots for parameters for two competing methods when the cluster size is only related to the cluster effect using the same parameters in Table 11-12. 
In Figure 5 and Figure 6, the cluster size was related to both cluster effect $\alpha_{i}$ and the cluster level covariate $Z_{i j 2}$. The parameters used are same as those in Table 13-14 and Table 15-16 respectively with censoring rate equal to 25 percent and the number of cluster equal to 200. The plots show that the cluster-weighted AFT model performed well for all parameters. However, the plots of both $\beta_{1}$ and $\beta_{2}$ are significantly deviated from the diagonal line for the non-cluster-weighted AFT model, indicating poor performance of the method.
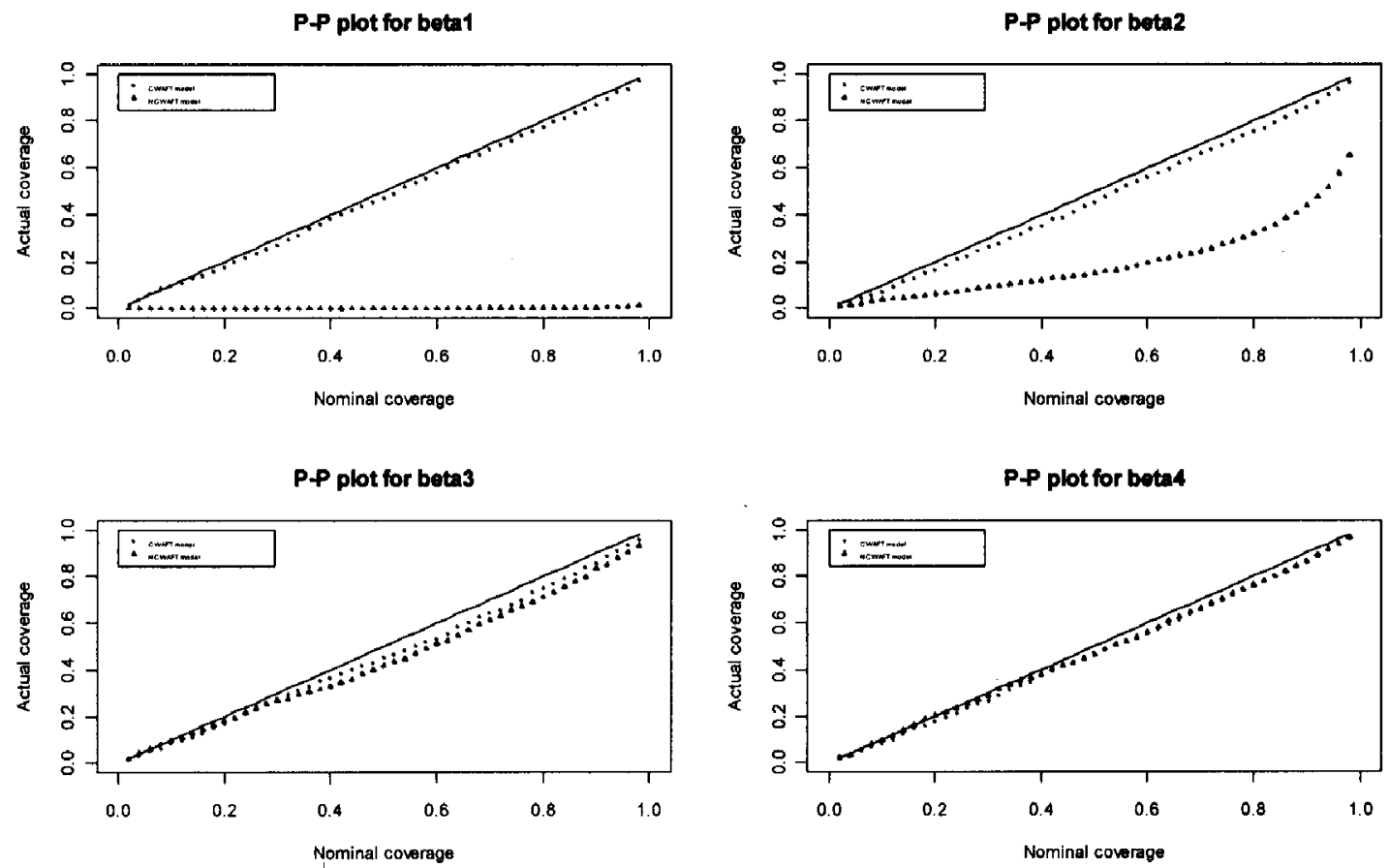

Figure 5. Probability-Probability plots for parameters for two competing methods when the cluster size is related to both the cluster effect and the covariate using the same parameters in Table 13-14. 

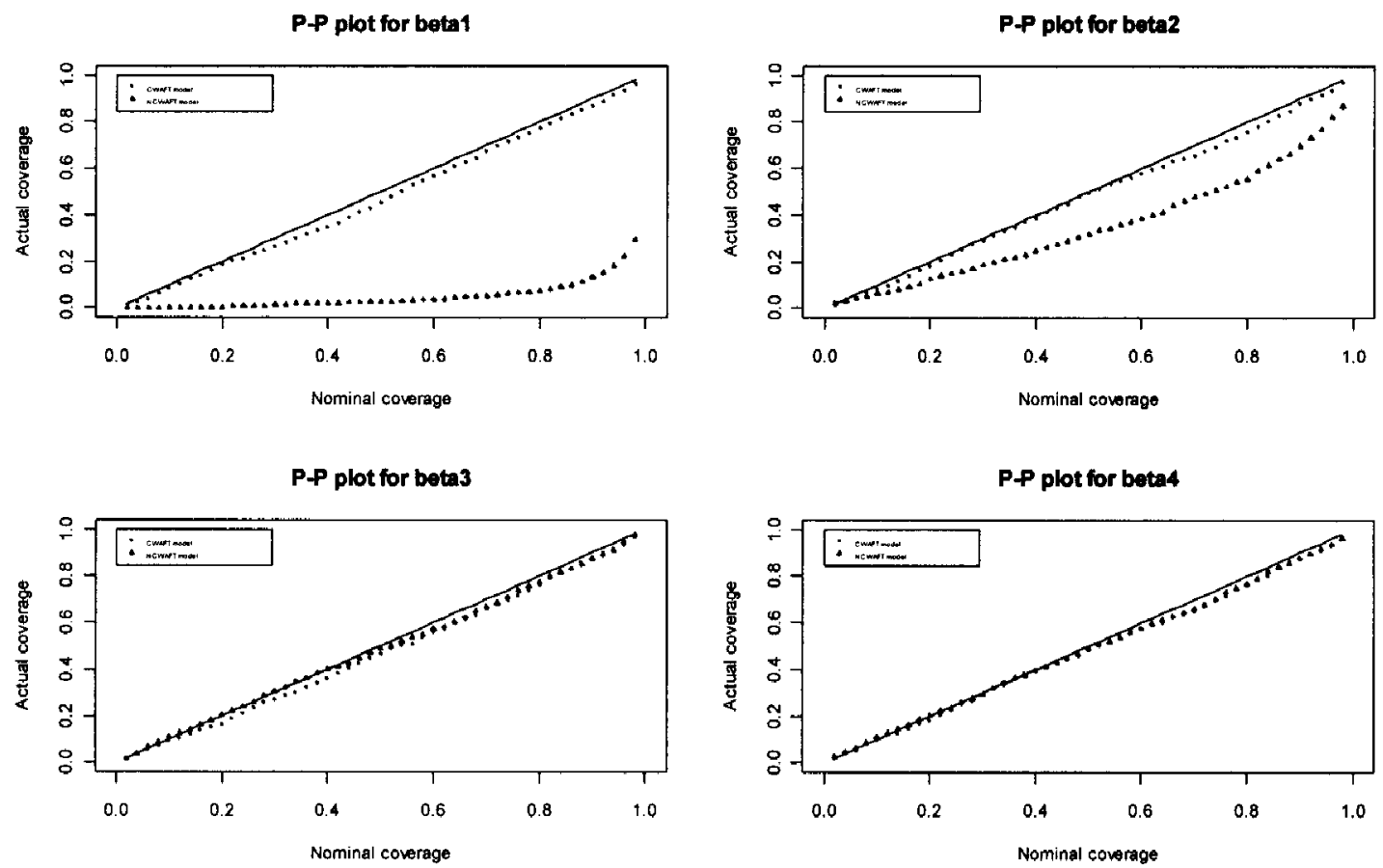

Figure 6. Probability-Probability plots for parameters for two competing methods when the cluster size is related to both the cluster effect and the covariate using the same parameters in Table 15-16.

We presented simulation results in Table 17 and Table 18 when cluster size is not informative. The results show when cluster size is not informative, the NCWAFT model is unbiased and the estimated variance is close to empirical variance indicating the model is appropriate. When cluster size is not informative, the CWAFT model continues to be unbiased with loss slight efficiency over NCWAFT model. 


\section{Table 17}

Simulation results for the cluster-weighted AFT model when the cluster size is not informative $($ Beta1 $=0.3 \mathrm{Beta} 2=0.4$, Beta3 $=-0.2$, Beta4 $=-0.3$, alpha $=0.25$, pai $=0.25$, $\mathrm{rl}=1.5, \mathrm{r} 2=3, \mathrm{z} 3=\mathrm{N}(1,0.15)$, error $=0.05)$.

\begin{tabular}{llrrr}
\hline Number of cluster & & \multicolumn{3}{c}{ Censoring rate } \\
& & 0.25 & \multicolumn{1}{c}{0.5} & 0.75 \\
\hline 30 & $\operatorname{Bias}\left(\widehat{\beta}_{1}\right)$ & 0.002 & 0.015 & 0.003 \\
& $\operatorname{e.sd}\left(\widehat{\beta}_{1}\right)$ & 0.257 & 0.328 & 0.469 \\
& $\operatorname{sd}\left(\widehat{\beta}_{1}\right)$ & 0.241 & 0.276 & 0.342 \\
& $\operatorname{Bias}\left(\widehat{\beta}_{2}\right)$ & -0.011 & -0.029 & -0.065 \\
& $\operatorname{e.sd}\left(\widehat{\beta}_{2}\right)$ & 0.401 & 0.544 & 0.818 \\
& $\operatorname{sd}\left(\widehat{\beta}_{2}\right)$ & 0.361 & 0.424 & 0.562 \\
& $\operatorname{Bias}\left(\widehat{\beta}_{3}\right)$ & 0.021 & 0.018 & 0.021 \\
& $\operatorname{e.sd}\left(\widehat{\beta}_{3}\right)$ & 0.245 & 0.316 & 0.461 \\
& $\operatorname{sd}\left(\widehat{\beta}_{3}\right)$ & 0.229 & 0.266 & 0.333 \\
& $\operatorname{Bias}\left(\widehat{\beta}_{4}\right)$ & 0.005 & 0.009 & 0.028 \\
& $\operatorname{e.sd}\left(\widehat{\beta}_{4}\right)$ & 0.381 & 0.521 & 0.791 \\
& $\operatorname{sd}\left(\widehat{\beta}_{4}\right)$ & 0.345 & 0.418 & 0.544 \\
& & & \\
& $\operatorname{Bias}\left(\widehat{\beta}_{1}\right)$ & 0.021 & 0.050 & 0.065 \\
& $\operatorname{e.sd}\left(\widehat{\beta}_{1}\right)$ & 0.112 & 0.145 & 0.245 \\
& $\operatorname{sd}\left(\widehat{\beta}_{1}\right)$ & 0.106 & 0.136 & 0.198 \\
& $\operatorname{Bias}\left(\widehat{\beta}_{2}\right)$ & -0.039 & -0.053 & -0.066 \\
& $\operatorname{e} . \operatorname{sd}\left(\widehat{\beta}_{2}\right)$ & 0.167 & 0.237 & 0.401 \\
& $\operatorname{sd}\left(\widehat{\beta}_{2}\right)$ & 0.161 & 0.217 & 0.318 \\
& $\operatorname{Bias}\left(\widehat{\beta}_{3}\right)$ & 0.005 & -0.004 & -0.011 \\
& $\operatorname{e.sd}\left(\widehat{\beta}_{3}\right)$ & 0.107 & 0.139 & 0.236 \\
& $\operatorname{sd}\left(\widehat{\beta}_{3}\right)$ & 0.102 & 0.132 & 0.193 \\
& $\operatorname{Bias}\left(\widehat{\beta}_{4}\right)$ & 0.026 & 0.026 & 0.030 \\
& $\operatorname{e.sd}\left(\widehat{\beta}_{4}\right)$ & 0.159 & 0.229 & 0.388 \\
$\operatorname{sd}\left(\widehat{\beta}_{4}\right)$ & 0.154 & 0.208 & 0.307 \\
\hline & & & &
\end{tabular}


Table 18

Simulation results for the non-cluster-weighted AFT model when the cluster size is not informative (Beta1 $=0.3$ Beta2 $=0.4$, Beta $3=-0.2$, Beta $4=-0.3$, alpha $=0.25$, pai $=0.25$, $\mathrm{r} 1=1.5, \mathrm{r} 2=3, \mathrm{z} 3=\mathrm{N}(1,0.15)$, error $=0.05)$.

\begin{tabular}{|c|c|c|c|c|}
\hline \multirow[t]{2}{*}{ Number of cluster } & & \multicolumn{3}{|c|}{ Censoring rate } \\
\hline & & 0.25 & 0.5 & 0.75 \\
\hline \multirow[t]{12}{*}{30} & $\operatorname{Bias}\left(\widehat{\beta}_{1}\right)$ & 0.012 & 0.024 & 0.009 \\
\hline & e.sd $\left(\widehat{\beta}_{1}\right)$ & 0.209 & 0.260 & 0.389 \\
\hline & $\operatorname{sd}\left(\widehat{\beta}_{1}\right)$ & 0.192 & 0.231 & 0.315 \\
\hline & $\operatorname{Bias}\left(\widehat{\beta}_{2}\right)$ & -0.014 & -0.032 & -0.069 \\
\hline & $\operatorname{e.sd}\left(\widehat{\beta}_{2}\right)$ & 0.317 & 0.440 & 0.717 \\
\hline & $\operatorname{sd}\left(\widehat{\beta}_{2}\right)$ & 0.295 & 0.378 & 0.518 \\
\hline & $\operatorname{Bias}\left(\widehat{\beta}_{3}\right)$ & 0.008 & 0.005 & 0.015 \\
\hline & $\operatorname{e.sd}\left(\widehat{\beta}_{3}\right)$ & 0.189 & 0.242 & 0.379 \\
\hline & $\operatorname{sd}\left(\widehat{\beta}_{3}\right)$ & 0.189 & 0.227 & 0.309 \\
\hline & $\operatorname{Bias}\left(\widehat{\beta}_{4}\right)$ & 0.012 & 0.019 & 0.037 \\
\hline & $\operatorname{e.sd}\left(\widehat{\beta}_{4}\right)$ & 0.289 & 0.413 & 0.692 \\
\hline & $\operatorname{sd}\left(\widehat{\beta}_{4}\right)$ & 0.288 & 0.368 & 0.504 \\
\hline \multirow[t]{12}{*}{200} & $\operatorname{Bias}\left(\widehat{\beta}_{1}\right)$ & 0.023 & 0.051 & 0.069 \\
\hline & $\operatorname{e.sd}\left(\widehat{\beta}_{1}\right)$ & 0.088 & 0.111 & 0.198 \\
\hline & $\operatorname{sd}\left(\widehat{\beta}_{1}\right)$ & 0.079 & 0.106 & 0.172 \\
\hline & $\operatorname{Bias}\left(\widehat{\beta}_{2}\right)$ & -0.035 & -0.051 & -0.066 \\
\hline & $\operatorname{e.sd}\left(\widehat{\beta}_{2}\right)$ & 0.130 & 0.182 & 0.324 \\
\hline & $\operatorname{sd}\left(\widehat{\beta}_{2}\right)$ & 0.121 & 0.174 & 0.284 \\
\hline & $\operatorname{Bias}\left(\widehat{\beta}_{3}\right)$ & 0.002 & -0.006 & -0.015 \\
\hline & $\operatorname{e.sd}\left(\widehat{\beta}_{3}\right)$ & 0.079 & 0.104 & 0.189 \\
\hline & $\operatorname{sd}\left(\widehat{\beta}_{3}\right)$ & 0.077 & 0.104 & 0.168 \\
\hline & $\operatorname{Bias}\left(\widehat{\beta}_{4}\right)$ & 0.023 & 0.026 & 0.032 \\
\hline & $\operatorname{e.sd}\left(\widehat{\beta}_{4}\right)$ & 0.119 & 0.174 & 0.314 \\
\hline & $\operatorname{sd}\left(\widehat{\beta}_{4}\right)$ & 0.118 & 0.169 & 0.274 \\
\hline
\end{tabular}


We also provide the Probability-Probability plots for the estimated regression coefficients in Figure 7 when cluster size is not informative. The parameters used are same as those in Table 17 and Table 18 with censoring rate equal to 25 percent and the number of cluster equal to 200 . The empirical coverage rates for both the CWAFT model and the NCWAFT model are close to the nominal level for all four parameters indicating that the both models perform well.
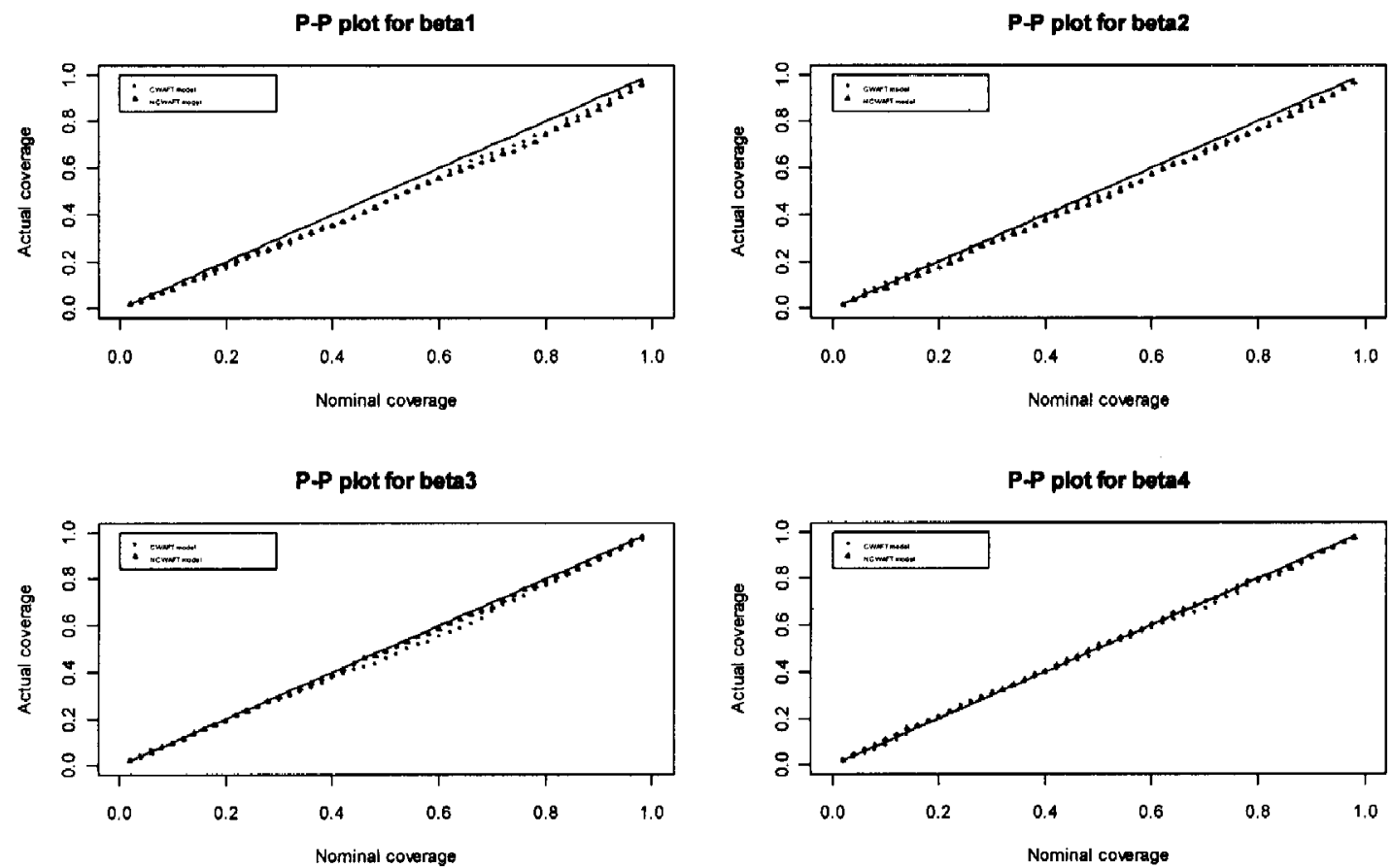

Figure 7. Probability-Probability plots for parameters for two competing methods when the cluster size is not informative using the same parameters in Table 17-18. 


\subsubsection{Testing Hypotheses for Cluster-weighted AFT Model}

From above theoretical examination and simulation results, we conclude that the CWAFT model worked well in presence of informative cluster size but the NCWAFT did not. Therefore, we conduct simulations for a power study using Wald tests only for the CWAFT model. We report here the results for the simulation scenario same with that for Table 5 with censoring rate equal to 25 and the number of cluster equal to 200 .

We studied power for $\beta_{1}$, where the hypotheses are: $H_{0}: \beta_{1}=0$ VS $H_{1}: \beta_{1} \neq 0$. We varied $\beta_{1}$ in $[-1,1]$ with a step of 0.05 while keeping the other parameters as same with those in the Table 5 with censoring rate equal to 25 percent. We simulated 1000 data sets. For each generated sample data set, the Wald test was applied. We calculated the percentage of the null hypothesis being rejected by Wald test, which is the power for the hypotheses. Figure 8 is the power curve for $\beta_{1}$. As shown in the plot, the empirically estimated size is 0.058 with confidence interval $(0.044,0.072)$, and the power increases gradually as $\beta_{1}$ leaves from zero. 


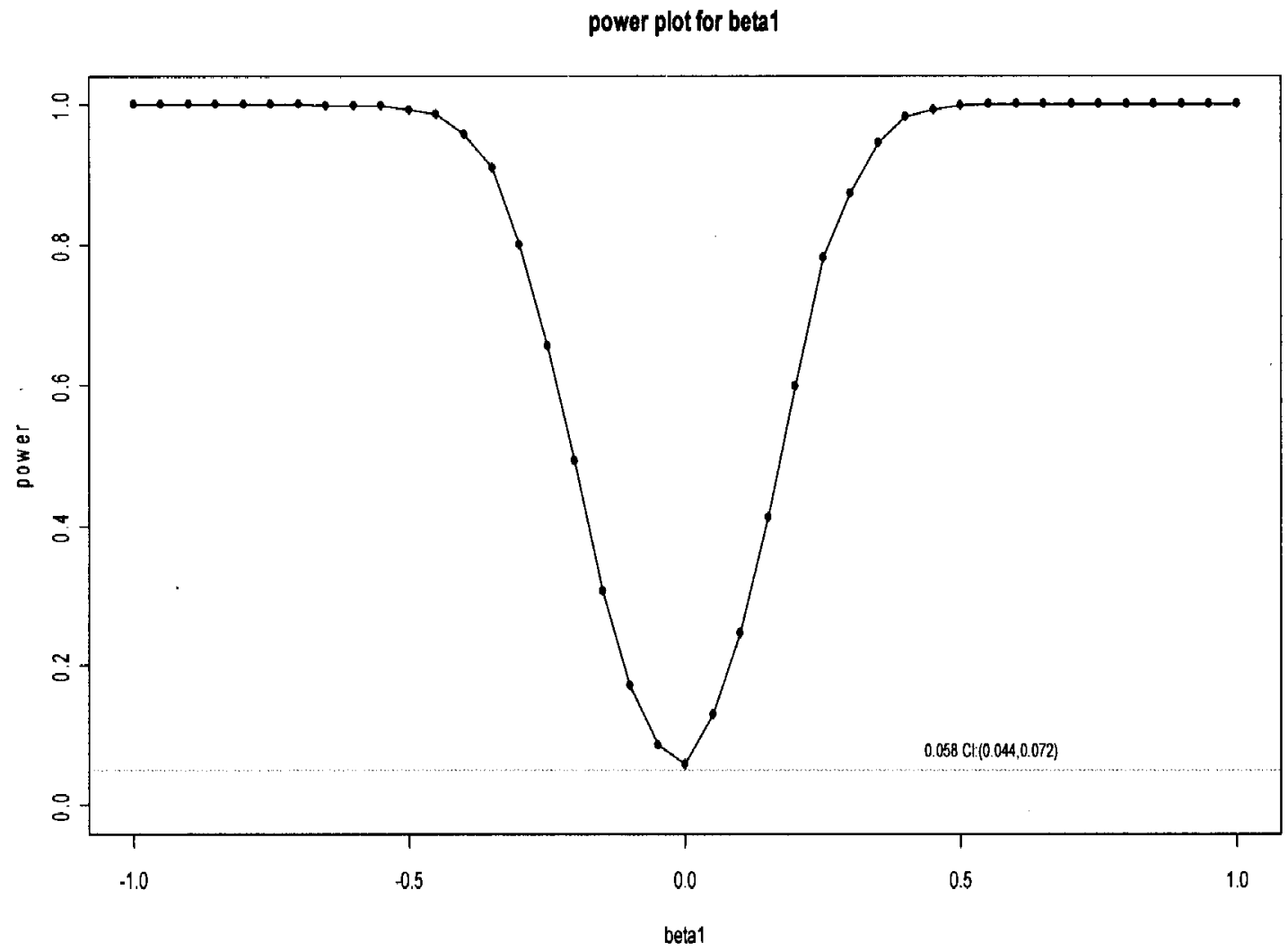

Figure 8. Power plot for $\beta_{1}$ when the cluster size is only related to the cluster effect. 


\subsection{An Application to Dental Study Data}

We applied our proposed CWAFT model to the dental study described in Spiekerman and Lin (1998). The dental study was originally conducted by McGuire and Nunn (1996). We obtained the data, a version of that study, from Dr. Nunn. Therefore, the data is different from the data in Spiekerman and Lin. This dental study was designed to assess the effect of commonly measured risk factors in predicting tooth survival. The original data set contains 2509 observations with 102 patients and 27 variables including age, smoke status, hygiene level, diabetic status, and etc. All of the patients had received at least 5 years of maintenance care. The outcome of interest is time to tooth loss from the beginning of the study. We used two risk factors, age and smoke status $(0=$ nonsmoker, $1=$ smoker), as predictor variables. Each patient is considered to be a cluster in this study. The measurement unit is each tooth of a patient. Patients with more teeth have higher molar survival probability. As a result, the cluster size in this study is informative to the outcomes of interest, time of tooth survival.

The cluster-weighted AFT model we used in the study was:

$$
E(Y)=\beta_{1}+\beta_{2} \text { smoke }+\beta_{3} \text { age }+\beta_{4} \text { smoke } * \text { age, }
$$

Table 19

Results for regression coefficients for the dental study.

\begin{tabular}{llrlr}
\hline Model & Predictor variable & Estimate & se & P-value \\
\cline { 2 - 5 } & Smoke & 1.631 & 0.638 & 0.005 \\
\cline { 2 - 5 } CWAFT & Age & 0.048 & 0.005 & 0.001 \\
\cline { 2 - 5 } & Smoke*Age & -0.044 & 0.018 & 0.008 \\
\cline { 2 - 5 } & Smoke & 1.694 & 0.479 & 0.001 \\
\cline { 2 - 5 } WCR & Age & -0.014 & 0.005 & 0.002 \\
\cline { 2 - 5 } & Smoke*Age & -0.008 & 0.005 & 0.053 \\
\cline { 2 - 5 } & & & &
\end{tabular}




\subsection{Discussion}

Clustered survival data with potentially informative cluster size are often encountered in medical research, however, few studies have been conducted in this area in the statistical literature. The traditional methods, represented by NCWAFT model in this research, do not take informative cluster size into account. We illustrate by simulation studies and by theoretical calculations that the estimated regression coefficients could be biased using the NCWAFT model in presence of informative cluster size and that the estimates by the CWAFT model are unbiased in the same situations. Simulation studies also show when cluster sizes are not informative, the CWAFT method continues to be unbiased with loss slight efficiency over NCWAFT method. We illustrate the use of the CWAFT method with the dental study data.

A marginal analysis is preferred in many situations due to its relative simplicity, while it is often sufficient for solving the problem. The strength of CWAFT method is that it does not need to estimate the parameters accounting for covariance between clusters. The effect of cluster effect can be cancelled out when estimating the coefficients of the covariates. 
CHAPTER III

\section{MANN-WHITNEY TEST FOR COMPARING SOJOURN TIME DISTRIBUTIONS WHEN TRANSITION TIMES ARE RIGHT CENSORED}

\subsection{Introduction}

The problem considered in this chapter is comparing sojourn time distributions of a transient state in a general multistate system in two groups when the transition times are right censored. Under this setup, the censoring can occur either in state entry or state exit.

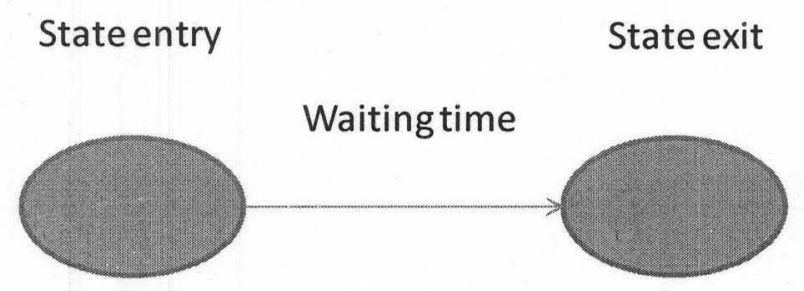

Figure 9. A two-state waiting time model.

The Mann-Whitney $U$ test (Mann \& Whitney, 1947) is perhaps the most often used nonparametric procedure in comparing two distributions based on independent samples. Procedurally, it is equivalent to Wilcoxon's rank-sum test and because of that test is sometimes collectively referred to as Wilcoxon-Mann-Whitney test. The test was 
proposed initially by Wilcoxon (1945), for equal sample sizes in two groups and later extended by Mann \& Whitney (1947) for possibly unequal sample sizes. Often statisticians regard the Mann-Whitney test as the non-parametric counterpart of the parametric two-sample $t$ test. The method is more robust than the $t$ test and can be applied to ordinal data in addition to continuous data. It is especially useful when the assumption of normality is not met.

However, the traditional Mann-Whitney $U$ test does not take missing values into account. In particular, in this research, we are interested in comparing the sojourn times in two independent samples when the transition times (e.g., both the state entry and the state exit times) are subject to right censoring. In this situation, missing data could arise if at least one of the state entry or exit times is right censored. None of the extensions of Mann-Whitney-Wilcoxon test to right censored data (Latta, 1977; Prentice, 1978) applies to this situation since the censoring induced on the set of waiting times is more complex than independent right censoring.

\subsection{Background}

The basic theory of $U$-statistics was developed by Hoeffding (1948). Denker (1985 ref) and Lee (1990 ref) discussed detailed expositions of the general topics. $U$-statistics are non-parametric method and they are generalizations of sample means. The $U$ statistics introduced here is based on uncensored data.

\subsubsection{One Sample $U$-statistic}

Let $X_{1}, \ldots, X_{n}$ be i.i.d with distribution $F$ on $\mathcal{X}\left(=\mathcal{R}^{p}\right.$, for some $\left.p \geq 1\right)$. Let $h$ : $\mathcal{X}^{m} \rightarrow \mathcal{R}$ be a measurable function, referred to as a kernel of degree $m$. The function $h$ is symmetric in its $m$ arguments and $E_{F}\left\{h^{2}\left(X_{1}, \ldots, X_{m}\right)\right\}<\infty$. 
Let $\theta_{F}=E_{F}\left\{h\left(X_{1}, \ldots, X_{m}\right)\right\}=\int \ldots \int h\left(x_{1}, \ldots, x_{m}\right) d F\left(x_{1}\right) \ldots d F\left(x_{m}\right)$ for which there is an unbiased estimator.

Let $P_{n m}$ be the collection of all $n ! /(n-m)$ ! permutations $\left(i_{1}, \ldots i_{m}\right)$ of size $m$ chosen from $(1, \ldots, n)$; i.e., $P_{n m}=\left\{\boldsymbol{i}:\left(i_{1}, \ldots i_{m}\right) \in N^{m}: 1 \leq i_{1}<\ldots<i_{m} \leq n\right\}$

A $U$-statistic with kernel $h$ is defined by averaging all the summands $h\left(X_{i_{1}}, \ldots, X_{i_{m}}\right)$, $i \in P_{n m}$, as

$$
U_{n}=\left(\begin{array}{c}
n \\
m
\end{array}\right)^{-1} \sum_{i \in P_{n m}} h\left(X_{i_{1}}, \ldots, X_{i_{m}}\right)
$$

Then $U_{n}$ is an unbiased estimator of $\theta_{F}$.

\subsubsection{Generalized $U$-statistic}

Now, we extend one sample $U$-statistic to multiple samples. Our attempts to extend the Mann-Whitney $U$-statistic to sojourn times under right censored transition times are based on its representation as a generalized $U$-statistic. Consider $k$ independent collections of independent observations $\left\{X_{1,1}, \ldots X_{n_{1}, 1}\right\}, \ldots,\left\{X_{1, k}, \ldots X_{n_{k}, k}\right\}$ from distributions $F_{1}, \ldots, F_{k}$ respectively. Let $\theta\left(F_{1}, \ldots, F_{k}\right)$ denote a parametric function for which there is an unbiased estimator, i.e., $\theta\left(F_{1}, \ldots, F_{k}\right)=E\left\{h\left(X_{1,1}, \ldots, X_{m_{1}, 1}, \ldots, X_{1, k}\right.\right.$, $\left.\left.\ldots, X_{m_{k}, k}\right)\right\}$. We call

$$
U_{n}=U_{n}(h)=\left(\prod_{j=1}^{k}\left(\begin{array}{c}
n_{j} \\
m_{j}
\end{array}\right)\right)^{-1} \sum_{c} h\left(X_{i_{1}(1), 1}, \ldots, X_{i_{1}\left(m_{1}\right), 1}, \ldots, X_{i_{k}(1), k}, \ldots, X_{i_{k}\left(m_{k}\right), k}\right) .
$$

an unbiased generalized $U$-statistic for estimation of $\theta\left(F_{1}, \ldots, F_{k}\right)$ of degree $m_{1}, \cdots, m_{k}$ based on kernel $h, 1 \leq i_{j}(1)<\cdots<i_{j}\left(m_{j}\right) \leq n_{j}$ denote a set of $m_{j}$ ordered elements

of the set $\left\{1, \ldots, n_{j}\right\}, 1 \leq j \leq k$, and $\sum_{c}$ denotes summation over all such combinations of indices. 
Datta, Bandyopadhyay, and Satten (2010) proposed an inverse probability of censoring weighted (IPCW) $U$-statistics for right censored data. We adopt similar reweighting principles to deal with missing waiting times. However, the current setup is more complicated for two reasons. First of all, unlike $U$-statistics, generalized $U$-statistics involve multiple groups and more importantly, the right censoring mechanism is operating on the transition times and not on the sojourn times.

Using the reweighting principle, a two sample Mann-Whitney type U-statistic is constructed that compares only the uncensored state sojourn times from the two distributions. A second Mann-Whitney type statistic is also constructed using a different reweighting that allows for comparison when one of the two sojourn times is either uncensored or singly censored.

\subsection{Mann-Whitney Type U-statistics for sojourn times in Presence of Censoring}

We begin this section with some notations that will be needed to describe our statistics. Suppose we have right censored entry and exit time data from 2 independent population (groups). Let $X_{i, j}^{*}$ and $V_{i, j}^{*}$ be the possibly unobserved (due to right censoring) state entry and exit times, respectively, for the $i$ th subject in the $j$ th group both of which are subject to right censoring by a common censoring time $C_{i, j}$ which is assumed to be independent of the pair $\left(X_{i, j}^{*}, V_{i, j}^{*}\right)$. Our observed data consist of the four tuples $\left(X_{i j}, \xi_{i j}, V_{i j}, \delta_{i j}\right), 1 \leq i \leq n_{j}, j=1,2$, where, $\quad X_{i, j}=\min \left(X_{i, j}^{*}, C_{i, j}\right), V_{i, j}=\min \left(V_{i, j}^{*}\right.$, $\left.C_{i, j}\right)$ are the (right) censored state entry and exit times and $\xi_{i, j}=I\left(X_{i, j}^{*} \leq C_{i, j}\right)$, $\delta_{i, j}=I\left(V_{i, j}^{*} \leq C_{i, j}\right)$ be the censoring indicators for the $i$ th subject in the $j$ th group. Let $W_{i, j}^{*}=V_{i, j}^{*}-X_{i, j}^{*}$ be the possibly unobserved sojourn times and we define $W_{i, j}=V_{i, j}-X_{i, j}$. Note that $W_{i, j}$ is computable from the observed data and equals $W_{i, j}^{*}$ if and only if $\delta_{i, j}=1$. Let $F_{W_{j}}$ be the sojourn time distribution function in group $j$. 
When we compare two sojourn time distributions with no missing observations, the Mann-Whitney $U$-statistic is given by

$$
U^{*}=\frac{1}{n_{1} n_{2}} \sum_{i_{1}=1}^{n_{1}} \sum_{i_{2}=1}^{n_{2}} I\left(W_{i_{1}, 1}^{*} \leq W_{i_{2}, 2}^{*}\right) .
$$

In the present context, we replace $W^{*}$ by the observed data quantities $W$ for each pair with both $\delta=1$; i.e., we only select the fully observed sojourn times from each group for comparison. In order to compensate for this selection bias, we re-weigh each summand by inverse of the selection probabilities conditional on the state exit times for such a sample pair leading to the following extension of Mann-Whitney statistic

$$
U_{1}=\frac{1}{n_{1} n_{2}} \sum_{i_{1}=1}^{n_{1}} \sum_{i_{2}=1}^{n_{2}} \frac{I\left(W_{i_{1}, 1} \leq W_{i_{2}, 2}\right) \delta_{i_{1}, 1} \delta_{i_{2}, 2}}{K_{1}\left(V_{i_{1}, 1}-\right) K_{2}\left(V_{i_{2}, 2}-\right)},
$$

where $K_{j}(t)=\operatorname{pr}\left\{C_{j}>t\right\}$ is the survival function of the censoring times in group $j$.

The following simple argument shows that indeed $U_{1}$ agrees with the full data Mann-Whitney statistic on the average:

$$
\begin{gathered}
E\left(U_{1}\right)=\frac{1}{n_{1} n_{2}} \sum_{i_{1}=1}^{n_{1}} \sum_{i_{2}=1}^{n_{2}} E\left[E\left\{\frac{I\left(W_{i_{1}, 1} \leq W_{i_{2}, 2}\right) \delta_{i_{1}, 1} \delta_{i_{2}, 2}}{K_{1}\left(V_{i_{1}, 1}-\right) K_{2}\left(V_{i_{2}, 2}-\right)} \mid X_{i_{1}, 1}^{*}, V_{i_{1}, 1}^{*}, X_{i_{2}, 2}^{*}, V_{i_{2}, 2}^{*}\right\}\right] \\
=\frac{1}{n_{1} n_{2}} \sum_{i_{1}=1}^{n_{1}} \sum_{i_{2}=1}^{n_{2}} E\left[E\left\{\frac{I\left(W_{i_{1}, 1}^{*} \leq W_{i_{2}, 2}^{*}\right) \delta_{i_{1}, 1} \delta_{i_{2}, 2}}{K_{1}\left(V_{i_{1}, 1}^{*}-\right) K_{2}\left(V_{i_{2}, 2}^{*}-\right)} \mid X_{i_{1}, 1}^{*}, V_{i_{1}, 1}^{*}, X_{i_{2}, 2}^{*}, V_{i_{2}, 2}^{*}\right\}\right] \\
=\frac{1}{n_{1} n_{2}} \sum_{i_{1}=1}^{n_{1}} \sum_{i_{2}=1}^{n_{2}} E\left[\frac{I\left(W_{i_{1}, 1}^{*} \leq W_{i_{2}, 2}^{*}\right)}{K_{1}\left(V_{i_{1}, 1}^{*}-\right) K_{2}\left(V_{i_{2}, 2}^{*}-\right)}\right. \\
\left.\times E\left\{I\left(C_{i_{1}, 1} \geq V_{i_{1}, 1}^{*}\right) I\left(C_{i_{2}, 1} \geq V_{i_{2}, 1}^{*}\right) \mid X_{i_{1}, 1}^{*}, V_{i_{1}, 1}^{*}, X_{i_{2}, 2}^{*}, V_{i_{2}, 2}^{*}\right\}\right] \\
=\frac{1}{n_{1} n_{2}} \sum_{i_{1}=1 i_{2}=1}^{n_{1}} \sum^{n_{2}} E\left\{\frac{I\left(W_{i_{1}, 1}^{*} \leq W_{i_{2}, 2}^{*}\right)}{K_{1}\left(V_{i_{1}, 1}^{*}-\right) K_{2}\left(V_{i_{2}, 2}^{*}-\right)} \operatorname{pr}\left(C_{i_{1}, 1} \geq V_{i_{1}, 1}^{*} \mid X_{i_{1}, 1}^{*}, V_{i_{1}, 1}^{*}\right)\right.
\end{gathered}
$$




$$
\left.\times \operatorname{pr}\left(C_{i_{2}, 1} \geq V_{i_{2}, 1}^{*} \mid X_{i_{2}, 2}^{*}, V_{i_{2}, 2}^{*}\right)\right\}
$$

by independence of two samples,

$$
=\frac{1}{n_{1} n_{2}} \sum_{i_{1}=1}^{n_{1}} \sum_{i_{2}=1}^{n_{2}} E\left\{\frac{I\left(W_{i_{1}, 1}^{*} \leq W_{i_{2}, 2}^{*}\right)}{K_{1}\left(V_{i_{1}, 1}^{*}-\right) K_{2}\left(V_{i_{2}, 2}^{*}-\right)} K_{1}\left(V_{i_{1}, 1}^{*}-\right) K_{2}\left(V_{i_{2}, 2}^{*}-\right)\right\},
$$

by independence of $C_{i j}$ and $\left\{X_{i j}^{*}, V_{i j}^{*}\right\}$,

$$
=\frac{1}{n_{1} n_{2}} \sum_{i_{1}=1}^{n_{1}} \sum_{i_{2}=1}^{n_{2}} E\left\{I\left(W_{i_{1}, 1}^{*} \leq W_{i_{2}, 2}^{*}\right)\right\}=E\left(U^{*}\right) .
$$

Note that $U_{1}$ is not a statistic in the strict sense of the word since it involves the population quantities $K_{1}$ and $K_{2}$. We estimate $K_{j}$ by the group specific Kaplan-Meier estimator $\widehat{K}_{j}$ for the censoring survival function. Thus, $\widehat{K}_{j}(t-)$ can be computed based on sample $j(=1,2)$ by the standard Kaplan-Meier formula where the roles of failure and censoring times are switched. Substituting $\widehat{K}_{j}$ in place of $K_{j}$ we get our first MannWhitney type statistic

$$
\widehat{U}_{1}=\frac{1}{n_{1} n_{2}} \sum_{i_{1}=1}^{n_{1}} \sum_{i_{2}=1}^{n_{2}} \frac{I\left(W_{i_{1}, 1} \leq W_{i_{2}, 2}\right) \delta_{i_{1}, 1} \delta_{i_{2}, 2}}{\widehat{K}_{1}\left(V_{i_{1}, 1}-\right) \widehat{K}_{2}\left(V_{i_{2}, 2}-\right)}
$$

The second generalized Mann-Whitney $U$-statistic we are about to propose allows for comparison of additional sojourn times even when they are not fully observed. Note that the indicator kernel $I\left(W_{i_{1}, 1}^{*} \leq W_{i_{2}, 2}^{*}\right)$ can be evaluated when $W_{i_{1}, 1}^{*}=W_{i_{1}, 1}$ is nonmissing and we can conclude $W_{i_{2}, 2}^{*}$ is larger than $W_{i_{1}, 1}$ from the fact that $W_{i_{2}, 2}$ is larger than $W_{i_{1}, 1}$. In other words, the second entry time $X_{i_{2}, 2}^{*}=X_{i_{2}, 2}$ has to be non-missing as well and the second censoring time is at least $W_{i_{1}, 1}+X_{i_{2}, 2}$. The probability of both of these events occurring together given $X_{i_{1}, 1}^{*}, V_{i_{1}, 1}^{*}, X_{i_{2}, 2}^{*}, V_{i_{2}, 2}^{*}$ is $K_{1}\left(V_{i_{1}, 1}^{*}-\right) K_{2}\left(W_{i_{1}, 1}^{*}+X_{i_{2}, 2}^{*}-\right)$ which is the same as $K_{1}\left(V_{i_{1}, 1}-\right) K_{2}\left(W_{i_{1}, 1}+X_{i_{2}, 2}-\right)$ 
on the set $\delta_{i_{1}, 1} \xi_{i_{2}, 2}=1$. Thus, we obtain our second generalization of Mann-Whitney sojourn times statistic

$$
\widehat{U}_{2}=\frac{1}{n_{1} n_{2}} \sum_{i_{1}=1}^{n_{1}} \sum_{i_{2}=1}^{n_{2}} \frac{I\left(W_{i_{1}, 1} \leq W_{i_{2}, 2}\right) \delta_{i_{1}, 1} \xi_{i_{2}, 2}}{\widehat{K}_{1}\left(V_{i_{1}, 1}-\right) \widehat{K}_{2}\left(W_{i_{1}, 1}+X_{i_{2}, 2}-\right)} .
$$

Its unbiasedness, with the true $K_{j}$ in place of $\widehat{K}_{j}$, can be established as before. We expect $\widehat{U}_{2}$ to be more efficient since it is based on non-zero scores on a larger number of pairs.

\subsection{Large Sample Properties}

We denote by $\theta$ the population quantity $P\left\{W_{1}^{*} \leq W_{2}^{*}\right\}$ estimated by the MannWhitney U-statistic. When both sojourn time distributions are equal, $\theta=1 / 2$, but, in general, $\theta \in[0,1]$.

We need to introduce the following counting processes notation. Let $N_{i, j}^{c}(t)=I\left(V_{i, j} \leq t, \delta_{i, j}=0\right) \quad$ be the counting processes of censoring, $Y_{i, j}(t)=I\left(V_{i, j} \geq t\right)$ be the "at-risk" processes, and $M_{i, j}^{c}(t)=N_{i, j}^{c}(t)-\int_{0}^{t} Y_{i, j}(u) d \Lambda_{j}^{c}(u)$ be the martingale of the censoring process defined with respect to the appropriate filtration for the two samples; here, $\Lambda_{j}^{c}$ is the cumulative hazard for censoring in the $j$ th group, $j=1,2$. Let $\bar{n}_{j}$ be the subdistribution function of the pair $\left(W_{j}, V_{j}\right)$ corresponding to $\delta_{j}=1, j=1,2$, and $\bar{n}_{3}$ be the subdistribution function of the pair $\left(W_{2}, X_{2}\right)$ corresponding to $\xi_{2}=1$,

$$
\begin{aligned}
& \bar{n}_{j}(w, v)=\operatorname{pr}\left\{W_{j} \leq w, V_{j} \leq v, \delta_{j}=1\right\}, j=1,2, \\
& \bar{n}_{3}(w, x)=\operatorname{pr}\left\{W_{2} \leq w, X_{2} \leq x_{2}, \xi_{2}=1\right\} .
\end{aligned}
$$

Consider the following univariate functions on $[0, \infty)$ :

$$
\omega_{1}(s)=\frac{1}{y_{1}(s)} \int I(v>s) \frac{S_{2}(w)}{K_{1}(v-)} d \bar{n}_{1}(w, v)
$$




$$
\begin{gathered}
\omega_{2}(s)=\frac{1}{y_{2}(s)} \int I(v>s) \frac{F_{1}(w-)}{K_{2}(v-)} d \bar{n}_{2}(w, v), \\
\omega_{3}(s)=\frac{1}{y_{2}(s)} \int \frac{I\left(w_{1}+x_{2}>s\right) I\left(w_{1}<w_{2}\right) d F_{1}\left(w_{1}\right) d \bar{n}_{3}\left(w_{2}, x_{2}\right)}{K_{2}\left(w_{1}+x_{2}-\right)}
\end{gathered}
$$

where $y_{j}(s)=\operatorname{pr}\left(V_{j} \geq s\right), j=1,2, s \geq 0$.

THEOREM 1. Under suitable regularity conditions (see the Appendix), as $n \rightarrow \infty$,

$\sqrt{ } n\left(\widehat{U}_{1}-\theta\right) \stackrel{d}{\rightarrow} N\left(0, \sigma_{1}^{2}\right)$, where, $\sigma_{1}^{2}=c_{1}^{-1} \operatorname{var}\left(S_{2}\left(W_{1}\right) \delta_{1} / K_{1}\left(V_{1}-\right)\right.$

$\left.+\int_{0}^{\infty} \omega_{1}(s) d M_{1}^{c}(s)\right)+c_{2}^{-1} \operatorname{var}\left(F_{1}\left(W_{2}-\right) \delta_{2} / K_{2}\left(V_{2}-\right)+\int_{0}^{\infty} \omega_{2}(s) d M_{2}^{c}(s)\right)$, and $\sqrt{ } n\left(\widehat{U}_{2}-\theta\right) \stackrel{d}{\rightarrow} N\left(0, \sigma_{2}^{2}\right)$, where, $\sigma_{2}^{2}=c_{1}^{-1} \operatorname{var}\left(S_{2}\left(W_{1}\right) \delta_{1} / K_{1}\left(V_{1}-\right)\right.$

$\left.+\int_{0}^{\infty} \omega_{1}(s) d M_{1}^{c}(s)\right) \quad+c_{2}^{-1} \operatorname{var}\left(F_{1}\left(W_{2}-\right) \xi_{2} / K_{2}\left(W_{1}+X_{2}-\right)\right.$

$\left.+\int_{0}^{\infty} \omega_{3}(s) d M_{2}^{c}(s)\right)$, with $c_{j}=\lim \left(n_{j} / n\right), j=1,2$.

The above expressions for the asymptotic variances also suggest the following natural estimators:

$$
\widehat{\sigma}_{1}^{2}=\frac{n}{n_{1}\left(n_{1}-1\right)} \sum_{i_{1}=1}^{n_{1}}\left(S_{i_{1}, 1}-\bar{S}_{1}\right)^{2}+\frac{n}{n_{2}\left(n_{2}-1\right)} \sum_{i_{2}=1}^{n_{2}}\left(S_{i_{2}, 2}-\bar{S}_{2}\right)^{2}
$$

where

$$
\begin{aligned}
S_{i, 1} & =\frac{\widehat{S}_{2}\left(W_{i, 1}\right) \delta_{i, 1}}{\widehat{K}_{1}\left(V_{i, 1}-\right)}+\widehat{\omega}_{1}\left(V_{i, 1}\right) \bar{\delta}_{i, 1}-\sum_{i_{1}=1}^{n_{1}} \frac{\widehat{\omega}_{1}\left(V_{i_{1}, 1}\right) I\left(V_{i, 1} \geq V_{i_{1}, 1}\right) \bar{\delta}_{i_{i}, 1}}{Y_{1}\left(V_{i_{1}, 1}\right)}, \\
S_{i, 2} & =\frac{\widehat{F}_{1}\left(W_{i, 2}\right) \delta_{i, 2}}{\widehat{K}_{2}\left(V_{i, 2}-\right)}+\widehat{\omega}_{2}\left(V_{i, 2}\right) \bar{\delta}_{i, 2}-\sum_{i_{2}=1}^{n_{2}} \frac{\widehat{\omega}_{2}\left(V_{i_{2}, 2}\right) I\left(V_{i, 2} \geq V_{i_{2}, 2}\right) \bar{\delta}_{i_{2}, 2}}{Y_{2}\left(V_{i_{2}, 2}\right)},
\end{aligned}
$$




$$
\begin{aligned}
& \bar{S}_{j}=n_{j}^{-1} \sum_{i=1}^{n_{j}} S_{i j}, \quad \bar{\delta}_{i, 1}=1-\delta_{i, j}, j=1,2, \text { and } \\
& \widehat{\omega}_{1}(s)=\frac{1}{Y_{1}(s)} \sum_{i=1}^{n_{1}} I\left(V_{i, 1}>s\right) \frac{\widehat{S}_{2}\left(W_{i, 1}\right) \delta_{i, 1}}{\widehat{K}_{1}\left(V_{i, 1}-\right)}, \\
& \widehat{\omega}_{2}(s)=\frac{1}{Y_{2}(s)} \sum_{i=1}^{n_{2}} I\left(V_{i, 2}>s\right) \frac{\widehat{F}_{1}\left(W_{i, 2}-\right) \delta_{i, 2}}{\widehat{K}_{2}\left(V_{i, 2}-\right)} .
\end{aligned}
$$

In (8) and (10) above, $\widehat{S}_{2}$ is the Satten-Datta estimator (Satten and Datta, 2002) of the survival function of $W_{2}^{*}$ based on sample 2, i.e.,

$$
\widehat{S}_{2}\left(W_{i, 1}\right)=\frac{1}{n_{2}} \sum_{i_{2}=1}^{n_{2}} \frac{I\left(W_{i_{2}, 2}>W_{i, 1}\right) \delta_{i_{2}, 2}}{\widehat{K}_{2}\left(V_{i_{2}, 2}-\right)}
$$

in (9) and (11), $\widehat{F}_{1}=1-\widehat{S}_{1}$ is the Satten-Datta estimator of the distribution function of $W_{1}^{*}$ based on sample 1, i.e.,

$$
\widehat{F}_{1}\left(W_{i, 2}\right)=\frac{1}{n_{1}} \sum_{i_{1}=1}^{n_{1}} \frac{I\left(W_{i_{1}, 1} \leq W_{i, 2}\right) \delta_{i_{1}, 1}}{\widehat{K}_{1}\left(V_{i_{1}, 1}-\right)} .
$$

Similarly, $\sigma_{2}^{2}$ can be estimated by

$$
\widehat{\sigma}_{2}^{2}=\frac{n}{n_{1}\left(n_{1}-1\right)} \sum_{i_{1}=1}^{n_{1}}\left(S_{i_{1}, 1}-\bar{S}_{1}\right)^{2}+\frac{n}{n_{2}\left(n_{2}-1\right)} \sum_{i_{2}=1}^{n_{2}}\left(S_{i_{2}, 3}-\bar{S}_{3}\right)^{2} .
$$

with

$$
\begin{gathered}
S_{i, 3}=\frac{\widehat{F}_{1}\left(W_{i, 2}\right) \xi_{i, 2}}{\widehat{K}_{2}\left(W_{i, 1}+X_{i, 2}-\right)}+\widehat{\omega}_{3}\left(V_{i, 2}\right) \bar{\xi}_{i, 2}-\sum_{i_{2}=1}^{n_{2}} \frac{\widehat{\omega}_{3}\left(V_{i_{2}, 2}\right) I\left(V_{i, 2} \geq V_{i_{2}, 2}\right) \bar{\xi}_{i_{2}, 2}}{Y_{2}\left(V_{i_{2}, 2}\right)}, \\
\bar{\xi}_{i, 2}=1-\xi_{i_{2}, 2},
\end{gathered}
$$

and

$$
\widehat{\omega}_{3}(s)=\frac{1}{Y_{2}(s)} \sum_{i_{1}=1}^{n_{1}} \sum_{i_{2}=1}^{n_{2}} \frac{\delta_{i_{1}, 1} \xi_{i_{2}, 2} I\left(W_{i_{1}, 1}+X_{i_{2}, 2}>s\right) I\left(W_{i_{1}, 1}<W_{i_{2}, 2}\right)}{\widehat{K}_{1}\left(V_{i_{1}, 1}-\right) \widehat{K}_{2}\left(W_{i_{1}, 1}+X_{i_{2}, 2}-\right)} .
$$


In simulation studies (Section 3.6), we observe that $\widehat{U}_{2}$ is more efficient than $\widehat{U}_{1}$, which was intuitively clear from the discussion in Section 3.3. Therefore, we only consider $\widehat{U}_{2}$ for constructing our test statistic in the following section.

\subsection{Testing the Equality of Sojourn Time Distributions in 2 Groups}

We now use the second Mann-Whitney type statistic $\widehat{U}_{2}$ for testing the equality of sojourn time distributions in 2 groups based on independent samples from these group in the presence of right censoring on the transition times. We assume that the sojourn time distributions are continuous. In this section, we denote by $\widehat{U}(1,2)$ the test-statistic $\widehat{U}_{2}$ given in (4) based on group 1 and group 2 samples in that order. Note that, for our censored setup, the statistics $\widehat{U}(1,2)$ and $1-\widehat{U}(2,1)$ will be close but not equal unless all sojourn times are non-missing for a sample. Therefore, we could take their average $T=0.5\{\widehat{U}(1,2)+1-\widehat{U}(2,1)\}$ as the (one sided) test statistic for testing the null hypothesis $H_{0}: F_{W_{1}^{*}}=F_{W_{2}^{*}}$ of equality of group 1 and 2 sojourn time distributions which has an asymptotic mean of $\theta=0.5$ under the null hypothesis. Following the same

linearizations as in the Theorem 1 , we can estimate its asymptotic variance by $n^{-1} \widehat{\sigma}_{H_{0}}^{2}$ given by

$$
\widehat{\sigma}_{H_{0}}^{2}=\frac{n}{4 n_{1}\left(n_{1}-1\right)} \sum_{i_{1}=1}^{n_{1}}\left(S_{i_{1}, 4}-\bar{S}_{4}\right)^{2}+\frac{n}{4 n_{2}\left(n_{2}-1\right)} \sum_{i_{2}=1}^{n_{2}}\left(S_{i_{2}, 5}-\bar{S}_{5}\right)^{2},
$$

where

$$
\begin{gathered}
S_{i, 4}=\frac{\widehat{S}_{2}\left(W_{i, 1}\right) \delta_{i, 1}}{\widehat{K}_{1}\left(V_{i, 1}-\right)}+\widehat{\omega}_{1}\left(V_{i, 1}\right) \bar{\delta}_{i, 1}-\sum_{i_{1}=1}^{n_{1}} \frac{\widehat{\omega}_{1}\left(V_{i_{1}, 1}\right) I\left(V_{i, 1} \geq V_{i_{1}, 1}\right) \bar{\delta}_{i_{i}, 1}}{Y_{1}\left(V_{i_{1}, 1}\right)} \\
-\frac{\widehat{F}_{2}\left(W_{i, 1}\right) \xi_{i, 1}}{\widehat{K}_{1}\left(X_{i, 1}+W_{i, 2}-\right)}-\widehat{\omega}_{4}\left(V_{i, 1}\right) \bar{\xi}_{i, 1}+\sum_{i_{1}=1}^{n_{1}} \frac{\widehat{\omega}_{4}\left(V_{i_{1}, 1}\right) I\left(V_{i, 1} \geq V_{i_{1}, 1}\right) \bar{\xi}_{i_{1}, 1}}{Y_{1}\left(V_{i_{1}, 1}\right)}
\end{gathered}
$$


and

$$
\begin{aligned}
S_{i, 5}= & \frac{\widehat{F}_{1}\left(W_{i, 2}\right) \xi_{i, 2}}{\widehat{K}_{2}\left(W_{i, 1}+X_{i, 2}-\right)}+\widehat{\omega}_{3}\left(V_{i, 2}\right) \bar{\xi}_{i, 2}-\sum_{i_{2}=1}^{n_{2}} \frac{\widehat{\omega}_{3}\left(V_{i_{2}, 2}\right) I\left(V_{i, 2} \geq V_{i_{2}, 2}\right) \bar{\xi}_{i_{2}, 2}}{Y_{2}\left(V_{i_{2}, 2}\right)} \\
& -\frac{\widehat{S}_{1}\left(W_{i, 2}\right) \delta_{i, 2}}{\widehat{K}_{2}\left(V_{i, 2}-\right)}-\widehat{\omega}_{5}\left(V_{i, 2}\right) \bar{\delta}_{i, 2}+\sum_{i_{2}=1}^{n_{2}} \frac{\widehat{\omega}_{5}\left(V_{i_{1}, 2}\right) I\left(V_{i, 2} \geq V_{i_{2}, 2}\right) \bar{\delta}_{i_{2}, 2}}{Y_{2}\left(V_{i_{2}, 2}\right)}
\end{aligned}
$$

with

$$
\widehat{\omega}_{4}(s)=\frac{1}{Y_{1}(s)} \sum_{i_{1}=1}^{n_{1}} \sum_{i_{2}=1}^{n_{2}} \frac{\xi_{i_{1}, 1} \delta_{i_{2}, 2} I\left(X_{i_{1}, 1}+W_{i_{2}, 2}>s\right) I\left(W_{i_{2}, 2}<W_{i_{1}, 1}\right)}{\widehat{K}_{1}\left(X_{i_{1}, 1}+W_{i_{2}, 2}-\right) \widehat{K}_{2}\left(V_{i_{2}, 2}-\right)}
$$

and

$$
\widehat{\omega}_{5}(s)=\frac{1}{Y_{2}(s)} \sum_{i=1}^{n_{2}} I\left(V_{i, 2}>s\right) \frac{\widehat{S}_{1}\left(W_{i, 2}\right) \delta_{i, 2}}{\widehat{K}_{2}\left(V_{i, 2}-\right)}
$$

THEOREM 2. Under the null hypothesis $H_{0}: F_{W_{1}^{*}}=F_{W_{2}^{*}}, \quad Z_{n_{1}, n_{2}}:=$ $\sqrt{ } n \widehat{\sigma}_{H_{0}}^{-1}(T-0.5) \stackrel{d}{\rightarrow} N(0,1)$, as $n \rightarrow \infty$, provided the regularity conditions of Theorem 1 hold.

An empirical power study of this test is carried out in the second part of Section 3.6 to investigate the performance of this test in small to moderate samples.

\subsection{Simulation Studies and Results}

We conducted a number of simulation studies for investigating the finite sample behaviors of the Mann-Whitney type statistics (Section 3.4) and the large sample test (Section 3.5) for the equality of two sojourn time distributions. 


\subsubsection{A Semi-Markov Model}

In this simulation scenario, we generated sojourn times independently of the state entry times. The same distributions were used in both groups. The state entry and the sojourn times were each generated from a standard log-normal distribution. The censoring times are also generated by lognormal distribution with unit scale parameter but with possibly different log-mean parameters in the two groups which were varied to achieve different censoring rates.

In all cases, equal samples sizes $\left(n_{j}=25\right.$ and 50$)$ in two groups were used. Table 20 reports the results when the same censoring rates were used in two groups. The common censoring rates varied from low (25\%) to heavy (75\%). A Monte Carlo size of 1000 was used to compute the answers reported in Table 20. 
Table 20

Simulation results for $U$-statistics when censoring rates are same in two groups.

\begin{tabular}{llrrr}
\hline Group size & & \multicolumn{3}{c}{ Censoring rate } \\
& & 0.25 & \multicolumn{1}{c}{0.5} & \multicolumn{1}{c}{0.75} \\
\hline 25 & $\operatorname{Bias}\left(\widehat{U}_{1}\right)$ & -0.011 & -0.038 & -0.096 \\
& $\operatorname{Bias}\left(\widehat{U}_{2}\right)$ & -0.016 & -0.042 & -0.105 \\
& $\operatorname{e.se}\left(\widehat{U}_{1}\right)$ & 0.099 & 0.132 & 0.197 \\
& $\operatorname{ess}\left(\widehat{U}_{2}\right)$ & 0.096 & 0.122 & 0.157 \\
& $\operatorname{se}\left(\widehat{U}_{1}\right)$ & 0.098 & 0.125 & 0.169 \\
& $\operatorname{se}\left(\widehat{U}_{2}\right)$ & 0.095 & 0.116 & 0.148 \\
& & & & \\
& $\operatorname{Bias}\left(\widehat{U}_{1}\right)$ & -0.005 & -0.019 & -0.060 \\
& $\operatorname{Bias}\left(\widehat{U}_{2}\right)$ & -0.008 & -0.024 & -0.060 \\
& $\operatorname{e.se}\left(\widehat{U}_{1}\right)$ & 0.070 & 0.094 & 0.139 \\
& $\operatorname{e} \operatorname{se}\left(\widehat{U}_{2}\right)$ & 0.068 & 0.086 & 0.119 \\
& $\operatorname{se}\left(\widehat{U}_{1}\right)$ & 0.069 & 0.089 & 0.126 \\
$\operatorname{se}\left(\widehat{U}_{2}\right)$ & 0.067 & 0.083 & 0.111 \\
\hline
\end{tabular}

From Table 20, it is evident that the variance formulas are working since the estimated standard errors are close to the empirical standard errors for both methods. Biases and standard errors increase for both methods when censoring rate increases and/or the group sample size decreases, as to be expected. We also find that the bias for $\widehat{U}_{1}$ is very slightly smaller than $\widehat{U}_{2}$ under this simulation scenario for the smaller sample size; however, the estimated standard error for $\widehat{U}_{1}$ is consistently larger than that for $\widehat{U}_{2}$. 


\section{Table 21}

Simulation results for $U$-statistics when censoring rates are different in two groups.

\begin{tabular}{llrrr}
\hline Group size & & \multicolumn{3}{c}{ Censoring rate 1/censoring rate 2 } \\
& & $0.25 / 0.5$ & $0.25 / 0.75$ & $0.5 / 0.75$ \\
\hline 25 & $\operatorname{Bias}\left(\widehat{U}_{1}\right)$ & -0.034 & -0.082 & -0.087 \\
& $\operatorname{Bias}\left(\widehat{U}_{2}\right)$ & -0.038 & -0.092 & -0.097 \\
& $\operatorname{e.se}\left(\widehat{U}_{1}\right)$ & 0.119 & 0.175 & 0.181 \\
& $\operatorname{e.se}\left(\widehat{U}_{2}\right)$ & 0.109 & 0.123 & 0.132 \\
& $\operatorname{se}\left(\widehat{U}_{1}\right)$ & 0.115 & 0.143 & 0.151 \\
& $\operatorname{se}\left(\widehat{U}_{2}\right)$ & 0.105 & 0.117 & 0.127 \\
& & & & \\
50 & $\operatorname{Bias}\left(\widehat{U}_{1}\right)$ & -0.017 & -0.052 & -0.053 \\
& $\operatorname{Bias}\left(\widehat{U}_{2}\right)$ & -0.023 & -0.052 & -0.053 \\
& $\operatorname{e.se}\left(\widehat{U}_{1}\right)$ & 0.086 & 0.122 & 0.128 \\
& $\operatorname{e.se}\left(\widehat{U}_{2}\right)$ & 0.077 & 0.096 & 0.104 \\
& $\operatorname{se}\left(\widehat{U}_{1}\right)$ & 0.082 & 0.107 & 0.113 \\
& $\operatorname{se}\left(\widehat{U}_{2}\right)$ & 0.075 & 0.088 & 0.095 \\
\hline
\end{tabular}

Results for different degree of censoring in the two groups are reported in Table 21. Comparing results in Table 20 and Table 21, we conclude that they have similar patterns for the bias and the standard error for both methods. Overall, the second statistics $\widehat{U}_{2}$ is a better choice for extending the Mann-Whitney statistic to the current setup involving right censoring on the transition times. 


\subsubsection{A Markov Model}

In this simulation, we generate entry times with in each group from a standard lognormal distribution. After obtaining an entry time $X^{*}$, say, the corresponding exit time was obtained by the formula

$$
V^{*}=D^{-1}\left[D\left(X^{*}\right)+U\left\{1-D\left(X^{*}\right)\right\}\right]
$$

where $D(\cdot)$ is the distribution function, or cumulative density function of lognormal distribution; $U$ is a number randomly generated from a uniform distribution in the interval $[0,1]$ and $D^{-1}$ denotes the quantile function of lognormal distribution. Note that this ensures that $V^{*} \geq U^{*}$; furthermore, the resulting system is Markov and the transition hazard for $V^{*}$ is also that of a standard log-normal.

The censoring times were generated by the same mechanism as in simulation for semi-Markov model. 
Table 22

Simulation results for $U$-statistics under Markov model.

\begin{tabular}{llrrr}
\hline Group size & & \multicolumn{3}{c}{ Censoring rate } \\
& & 0.25 & \multicolumn{1}{c}{0.5} & \multicolumn{1}{c}{0.75} \\
\hline 25 & $\operatorname{Bias}\left(\widehat{U}_{1}\right)$ & -0.015 & -0.047 & -0.123 \\
& $\operatorname{Bias}\left(\widehat{U}_{2}\right)$ & -0.019 & -0.049 & -0.113 \\
& $\operatorname{e.se}\left(\widehat{U}_{1}\right)$ & 0.097 & 0.136 & 0.200 \\
& $\operatorname{e.se}\left(\widehat{U}_{2}\right)$ & 0.094 & 0.116 & 0.158 \\
& $\operatorname{se}\left(\widehat{U}_{1}\right)$ & 0.098 & 0.125 & 0.168 \\
& $\operatorname{se}\left(\widehat{U}_{2}\right)$ & 0.094 & 0.114 & 0.146 \\
& & & & \\
50 & $\operatorname{Bias}\left(\widehat{U}_{1}\right)$ & -0.009 & -0.029 & -0.082 \\
& $\operatorname{Bias}\left(\widehat{U}_{2}\right)$ & -0.010 & -0.029 & -0.072 \\
& $\operatorname{e.se}\left(\widehat{U}_{1}\right)$ & 0.066 & 0.090 & 0.146 \\
& $\operatorname{e.se}\left(\widehat{U}_{2}\right)$ & 0.065 & 0.082 & 0.117 \\
& $\operatorname{se}\left(\widehat{U}_{1}\right)$ & 0.068 & 0.089 & 0.127 \\
& $\operatorname{se}\left(\widehat{U}_{2}\right)$ & 0.067 & 0.082 & 0.111 \\
\hline
\end{tabular}

Once again, the estimated standard errors are close to their population counterparts; both bias and standard error decrease with the sample size and increase with censoring percentage. There is no consistent comparative pattern for the biases but the estimated standard error for $\widehat{U}_{2}$ is still consistently smaller than that for $\widehat{U}_{1}$. 


\subsubsection{A Model with Dependent Sojourn Times}

In this simulation scenario, sojourn times and the state entry times are dependent. The state entry and the sojourn times were generated from a multivariate log-normal distribution. The same distribution was used in both groups. The censoring times were generated by a lognormal distribution with unit scale parameter but with possible different log-mean parameters in the two groups which were varied to achieve different censoring rates.

\section{Table 23}

Simulation results for $U$-statistics when sojourn times and the state entry times are dependent.

\begin{tabular}{llrrr}
\hline Group size & & \multicolumn{3}{c}{ Censoring rate } \\
& & 0.25 & \multicolumn{1}{c}{0.5} & \multicolumn{1}{c}{0.75} \\
\hline 25 & $\operatorname{Bias}\left(\widehat{U}_{1}\right)$ & -0.012 & -0.034 & -0.098 \\
& $\operatorname{Bias}\left(\widehat{U}_{2}\right)$ & -0.017 & -0.043 & -0.103 \\
& $\operatorname{e.se}\left(\widehat{U}_{1}\right)$ & 0.073 & 0.112 & 0.191 \\
& $\operatorname{e} \mathrm{se}\left(\widehat{U}_{2}\right)$ & 0.066 & 0.098 & 0.147 \\
& $\operatorname{se}\left(\widehat{U}_{1}\right)$ & 0.098 & 0.125 & 0.168 \\
& $\operatorname{se}\left(\widehat{U}_{2}\right)$ & 0.096 & 0.115 & 0.149 \\
& & & & \\
& $\operatorname{Bias}\left(\widehat{U}_{1}\right)$ & -0.006 & -0.023 & -0.063 \\
& $\operatorname{Bias}\left(\widehat{U}_{2}\right)$ & -0.008 & -0.024 & -0.059 \\
& $\operatorname{e.se}\left(\widehat{U}_{1}\right)$ & 0.049 & 0.082 & 0.133 \\
& $\operatorname{e.se}\left(\widehat{U}_{2}\right)$ & 0.046 & 0.070 & 0.110 \\
& $\operatorname{se}\left(\widehat{U}_{1}\right)$ & 0.069 & 0.089 & 0.124 \\
& $\operatorname{se}\left(\widehat{U}_{2}\right)$ & 0.067 & 0.083 & 0.110 \\
\hline
\end{tabular}

Results for different degrees of censoring in the two groups are reported in Table 23. Comparing results in Table 23 with Table 20, we again conclude that they have similar patterns for the bias and the standard error for both methods. The second statistic $\widehat{U}_{2}$ is a better choice, especially, when the group size is large enough. 


\subsubsection{Testing hypotheses for equality of sojourn times}

We also conducted simulations for a power study using the test $Z_{n_{1}, n_{2}}$ described in Section 3.5. We varied logmean in $[-1.5,1.5]$ with a step of 0.1 in group 2 while keeping the other parameters as same with those in Table 20 with censoring rate equal to 25 percent; in particular, the group 1 sojourn times were generated from a standard lognormal distribution. To reduce computational burden, we compute the power at fewer values when the common group size was 50 . We simulated 1000 data sets under each parameter setting. For each generated sample data set, the studentized test statistic $Z_{n_{1}, n_{2}}$ was applied. The empirical power of the test in at each alternative parameter setting was calculated by the proportion of times the null hypothesis was rejected by this test out of 1000 samples. Figure 9 displays the resulting power curves. The empirically estimated sizes were 0.059 with a confidence interval $(0.044,0.074)$, for $n_{j} \equiv 25$ and 0.049 with a confidence interval $(0.036,0.062)$, for $n_{j} \equiv 50$, indicating that the targeted normal size is achieved by this large sample test. The power increased gradually as the group 2 logmean parameter shifted away from zero (the null). 


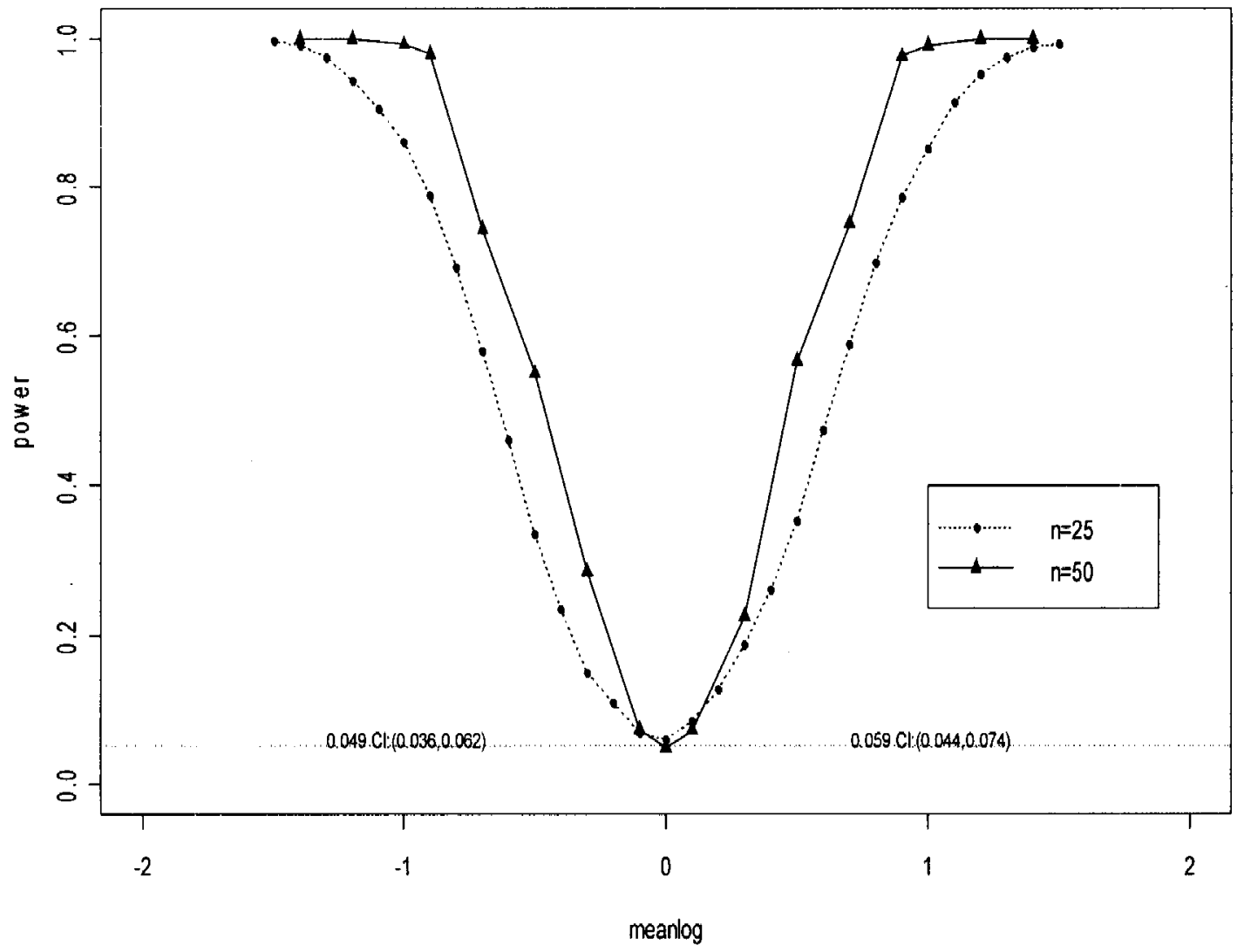

Figure 10. Power plot for the proposed generalized Mann-Whitney method. 


\subsection{An Illustration Using Kidney Disease Data}

McGilchrist \& Aisbett (1991) reported a study on recurrent events of infections of 38 kidney patients who are using a potable dialysis machine. Two times to recurrence of an infection were recorded as $T_{1}$ and $T_{2}$ for each patient, $\delta_{1}$ and $\delta_{2}$ were also recorded as the event indicators. The data contained a number of covariates including gender.

Considering this as a staged system where the first infection corresponds to state entry and the second infection corresponds to state exit, we can apply the test developed in Section 3.5. The range of the observed sojourn times was from 7 to 152 days for the male and from 5 to 511 for the female, respectively. About $29 \%$ of $T_{1}$ and $18 \%$ of $T_{2}$ were right censored. Overall, $20 \%$ of all sojourn times were missing for the male patients and $46 \%$ of all sojourn times were missing for female.

First of all, we are interested to determine if the gender has an impact on kidney disease recurrence time. For these data, the statistic $T$ defined in Section 3.5 turned out to be 0.216 with a null standard error of 0.106 . Using a two-sided $Z$ test, we obtain a pvalue of 0.042 . Thus, we conclude that gender may play a role on the recurrence time of infections for the study population. In particular, females appear to have a longer recurrence time.

Now, we want to investigate whether patients with the kidney disease type GN, AN, and PKD has different kidney disease recurrence time with patients without these three types of kidney disease. The $T$-statistic is calculated by our method as 0.024 , and the standard error is 0.107 . Using two-side $Z$ test, we get p-value 0.823 . We conclude that the combined factor with these three types of kidney diseases is not a significant factor on kidney disease recurrence time. These results are consistent with the findings of McGilchrist \& Aisbett (1991) who analyzed the data using a Cox type model with a multiplicative frailty term. 


\subsection{Discussion}

Traditional approaches of analyzing event time data include semi-parametric regression models such as the proportional hazards model which lead to appropriate estimating equations in presence of right censored data. A two sample comparison amounts to testing the effect of a single binary covariate on event time and the resulting test that arises out of a Cox model is the log-rank test. However, in many applications, time is measured since an initiating event rather than the calendar time. In other words, we may be dealing with a sojourn time and the standard estimating equations do not hold since the censoring will not be independent on the sojourn times. Furthermore, for some samples even the state entry time may be censored.

The methodology developed in this research is based on a novel reweighting scheme that extends the notion of a Mann-Whitney statistic to the present set-up where we are capable of making use of pairs of observable sojourn times plus additional pairs where one of the sojourn times may be missing since the exit time is right censored. The resulting statistic has desirable large sample properties including a closed form variance estimate. The large sample inference is fairly effective in moderate samples as well as shown by the simulation studies. The test may be extended to a multi-group comparison in a number of standard ways such as by considering suitable linear combinations, taking a maxima or by a quadratic form of pair specific test scores. 


\section{CHAPTER IV}

\section{EXTENSIONS AND FUTURE RESEARCH}

In chapter 2, we show that CWAFT model is unbiased method in presence of informative cluster size. In this research, we only consider the simplest working correlation matrix, the identity matrix. In the future, we will investigate CWAFT model with other correlation structures, such as exchangeable, AR-M and Tri-diagonal.

The covariates in our models are assuming independent of time in this dissertation research. We will also extend estimation for the CWAFT model to time dependent covariates. To that end, we need to understand the AFT model from another perspective. We will introduce estimation of an AFT model, proposed by Robins and Tsiatis (1992), for i.i.d. survival data with possibly time dependent covariates.

The AFT model for time independent covariates is

$$
Y=\beta_{0}+\boldsymbol{Z}^{\prime} \boldsymbol{\beta}+\varepsilon,
$$

where $\boldsymbol{Z}=\left(Z_{1}, \ldots Z_{p}\right)$ is a $p$-vector of time independent covariates, and $\boldsymbol{\beta}^{\prime}=\left(\beta_{1}, \ldots \beta_{p}\right)$ is a vector of regression coefficients.

Let $S_{0}(u)$ denote the survival function of $U=e^{Y}$ when $\boldsymbol{Z}$ is zero. So, $S_{0}(u)$ is the survival function of $\exp \left(\beta_{0}+\varepsilon_{i}\right)$, also called baseline survival function. 
The survival function for survival time $T$ is

$$
\begin{aligned}
\operatorname{Pr}(T>t \mid \boldsymbol{Z})= & \operatorname{Pr}(Y>\ln (t) \mid \boldsymbol{Z}) \\
& =\operatorname{Pr}\left(\beta_{0}+\varepsilon>\ln (t)-\boldsymbol{Z}^{\prime} \boldsymbol{\beta} \mid \boldsymbol{Z}\right) \\
& =\operatorname{Pr}\left(e^{\left(\beta_{0}+\varepsilon\right)}>t \exp \left(-\boldsymbol{Z}^{\prime} \boldsymbol{\beta}\right) \mid \boldsymbol{Z}\right) \\
& =S_{0}\left(\exp \left(-\boldsymbol{Z}^{\prime} \boldsymbol{\beta}\right)\right) .
\end{aligned}
$$

$\exp \left(-\boldsymbol{Z}^{\prime} \boldsymbol{\beta}\right)$ is the scale factor by which lifetime is either decrease or increase as a function of the covariates $\boldsymbol{Z}$ dependent on the sign of $\boldsymbol{Z}^{\prime} \boldsymbol{\beta}$. That is the reason why the model is so called accelerated failure time model. We will generalize this concept to time dependent covariates.

Let $\overline{\boldsymbol{Z}}_{i}(t)=\left\{\boldsymbol{Z}_{i}(s) ; 0 \leq s \leq t\right\}$ be the history of the covariate process through time $t$. The model assumes that there is a monotone transformation from the baseline time scale, $u$, to the observed time scale, $t$, as a function of the covariate history. It is convenient to consider the derivative of the transformation function, $d u / d t$, to be a function of the covariate history up to time $t, d u / d t=h\{\overline{\boldsymbol{Z}}(t), \boldsymbol{\beta}\}=e^{\boldsymbol{Z}^{\prime}(t) \boldsymbol{\beta}}$.An estimating equation is formed as:

$$
S(\beta)=\sum_{i=1}^{n} \delta_{i}\left\{G_{i}(\beta)-G^{a v}(\beta)\right\}
$$

where $G_{i}$ are suitable scoring functions and $G^{a v}$ is the average of the scores evaluated at changed time among the individuals who on the baseline scale are at risk at that time.

We will work on the details of obtaining the estimators of the parameters for cluster survival data, and study the finite sample properties of the estimators such as bias and standard error under simulations.

In chapter 3, we discuss the Mann-Whitney type U- statistics for sojourn times under right censoring, and the testing of the equality of sojourn time distributions in 2 groups. In the future research, we will extend the testing to multiple groups rather than 2 groups. We will also modify the U-statistic and its variance for time to event data so that we can conduct simulations comparing our method with log-rank test. 


\section{REFERENCES}

Aalen, O. O. (1978). Nonparametric inference for a family of counting processes. Annals of Statistics 6, 701-726.

Aalen, O. O. (1989). A linear regression model for the analysis of lifetimes. Statistics in Medicine. 8, 907-925.

Aalen, O. O. (1992). Modeling heterogeneity in survival analysis by the compound Poisson distribution. Annals of Applied Probability. 4, 951-972.

Andersen, P. K., Borgan, O., Gill, R. D. \& Keiding, N. (1993). Statistical Models Based on Counting Processes. New York: Springer.

Benhin, E., Rao, N.K., and Scott, A.J. (2005). Mean estimating equation approach to analysing cluster-correlated data with nonignorable cluster sizes. Biometrika. 92, 435-450.

Clayton, D. G. (1978) A model for association in bivariate life tables and its application in epidemiological studies of familial tendency in chronic disease incidence.

Biometrika. 65, 141-151.

Cong, X. J., Yin, G., and Shen, Y. (2007). Marginal analysis of correlated failure time data with informative cluster sizes. Biometrics. 63, 663-672.

Cox, D. R. (1972). Regression Model and Life Tables (with Discussion). Journal of the Royal Statistical Society. 34, 187-220.

Datta, S. (2005). Estimating the Mean Life Time Using Right Censored Data. Statistical Methodology. 2, 65-69.

Datta, S., Bandyopadhyay, D. and Satten, G. A. (2010). Inverse probability of censoring weighted U-statistics for right censored data with applications. Scandinavian Journal of Statistics, in press. 
Datta, S. and Satten, G. A. (2002). Estimation of integrated transition hazards and stage occupation probabilities for non-Markov systems under stage dependent censoring. Biometrics. 58, 792-802.

Datta, S., and Satten, G. A. (2008). A sign-rank test for clustered data. Biometrics. 64, 501-507.

Ding, J., and Wang J. (2008). Modeling longitudinal data with nonparametric multiplicative random effects jointly with survival data. Biometrics. 64, 546-556.

Dobson A. J. (2002). An introduction to generalized linear models. Second Edition. Chapman \& Hall/CRC.

Follmann, D., Proschan, M., and Leifer, E. (2003). Multiple outputation: Inference for complex clustered data by averaging analysis from independent data. Biometrics. $59,420-429$.

Hoeffding, W. (1948). A class of statistics with asymptotically normal distribution. Annals of Mathematic Statistics. 19, 293-325.

Hoffman, E,, Sen, P., and Weinberg, C. (2001). Within-cluster resampling. Biometrika. $88,1121-1134$.

Hougaard, P. (2000). Analysis of multivariate survival data. Springer, New York.

Huster, W. J., Brookmeyer, R., and Self, S.G. (1989). Modeling paired survival data with covariates. Biometrics. 45, 145-156.

Kaplan, E. L., and Meier, P. (1958). Nonparametric Estimation from Incomplete Observations. Journal of the American Statistical Association. 53, 457-481.

Klein, J. P., and Moeschberger, M. L. (2003). Survival Analysis: Techniques for Censored and Truncated Data. Second Edition. Springer. New York.

Koul, H., Susarla, Y., and Van Ryzin, J. (1981). Regression analysis with randomly right censored data. Annals of Statistics. 9, 1276-1288.

Lambert, P., Collett, D., Kimber, A., and Johnson, R. (2004). Parametric accelerated failure time models with random effects and an application to kidney transplant survival. Statistics in Medicine. 23, 3177-3192.

Lee, A. J. (1990). U-Statistics, Theory and Practice. Marcel Dekker, New York.

Lee, E. W., Wei, 1. J., and Amato, D. A. (1992). Cox-type regression analysis for large numbers of small groups of correlated failure time observations. In Survival 
Analysis: State of the Arts, Klein, J. P. and Goel, P.K. (eds). Dordrecht: Kluwer Academic Publishers. 237-247.

Lin, D. Y., Sun, W., and Ying, Z. (1999). Nonparametric estimation of the gap time distributions for serial events with censored data. Biometrika. 86, 59-70.

Mann, H. B., and Whitney, D. R. (1947). On a test of whether one of two random variables is stochastically larger than the other. Annals of Mathematical Statistics. $18,50-60$.

McCullagh, P., and Neldre, J. A. (1989), Generalized linear models. Second Edition. Chapman \& Hall.

McGilchrist, C. A., and Aisbett, C. W. (1991). Regression with frailty in survival analysis. Biometrics. 47, 461-466.

McGuire, M. K., and Nunn, M. E. (1996). Prognosis versus actual outcome III: The effects of clinical parameters in accurately predicting tooth survival. Journal of Periodontology. 67, 666-674.

Pan, W. (2001). Using frailties in the accelerated failure time model. Life Time Data Analysis. 7, 55-64.

Robins, J. M. and Rotnitzky (1992). Recovery of information and adjustment for dependent censoring using surrogate markers. In AIDS Epidemiology-Methodological Issues, eds. N. Jewell, K. Dieta, V. Farewell, Boston: Birkhauser. pp. 297-331.

Robins, J. M. (1992). Estimation of the time-dependent accelerated failure time model in the presence of confounding factors. Biometrika. 79, 2, 321-334.

Robins, J. M. (1993). Information recovery and bias adjustment in proportional hazards regression analysis of randomized trials using surrogate markers. Proceedings of the American Statistical Association-Biopharmaceutical Section, pp. 24-33.

Rotnitzky, A. and Robins, J. M. (2005). Inverse probability weighted estimation in survival analysis. In Encyclopedia of Biostatistics, 2nd Edition,eds. P.Amitage and T. Colliton. New York: Wiley.

Satten, G. A., and Datta, S. (2001). The Kaplan-Meier estimator as an inverseprobability-of-censoring weighted average. American Statistician. 55, 207-210.

Satten, G. A., Datta, S., and Robins, J.M. (2001). Estimating the marginal survival function in the presence of time dependent covariates. Statistics \& Probability Letters. 54, 397-403. 
Satten, G. A., and Datta, S. (2002). Marginal estimation for multi-stage models: waiting time distributions and competing risks analyses. Statistics in Medicine. 21, 3-19.

Saville, B. R., Herring A. H., and Koch, G. G. (2008). Analyzing correlated longitudinal and survival data in clinical trials using multivariate time-to event methods. The working paper hosted by the Berkeley Electronic Press.

Shih, W. J. (2002). Problems in dealing with missing data and informative censoring in clinical trials. Current Controlled Trials in Cardiovascular Medicine. 3:4.

Spiekerman, C., and Lin, D. Y. (1998). Marginal regression models for multivariate failure time data. Journal of the American Statistical Association. 93, 1164-1175.

Vaupel, J. W., Manton, K. G., Stallard, E. (1979). The impact of heterogeneity in individual frailty on the dynamics of mortality. Deemography. 16, 439-454.

Wang, M. C. M., Lam, K. F., and Lo, E. C. M. (2006). Multilevel modeling of clustered grouped survival data using Cox regression model: an application to ART dental restorations. Statistics in Medicine. 25, 447-457.

Wang, M., Kong, M., and Datta, S. Inference for marginal linear models with clustered longitudinal data with potentially informative cluster sizes. (2009) Statistical Methods in Medical Research, in press.

Wang, W. and Wells, M.T. (1998). Nonparametric estimation of successive duration times under dependent censoring. Biometrika. 85, 561-572.

Wei, L. J., Lin, D. Y., and Weissfeld, L. (1989). Regression analysis of multivariate incomplete failure time data by modeling marginal distributions. Journal of the American Statistical Association. 84, 1065-1073.

Wei, L.J., Ying, Z., and Lin, D. Y. (1990). Linear regression analysis of censored survival data based on rank test. Biometrika. 77, 845-851.

Wei, L. J. (1992). The accelerated failure time model: a useful alternative to the Cox regression model in survival analysis. Statistics in Medicine. 11, 1871-1879.

Wienke, A., Arbeev, K., Locatelli, I., and Yashin, A. I. (2003). A simulation study of different correlated frailty models and estimation strategies. MPIDR working paper wp 2003-018.

Wilcoxon, F. (1945). Individual comparisons by ranking methods. Biometrics. 1, 80-83.

Williamson, J. M., Datta, S., and Satten, G. A. (2003). Marginal analyses of clustered 
data when cluster size is informative. Biometrics. 59, 36-42.

Williamson, J. M., Kim, H., Manatunga, A., and Addiss, D. G. (2008). Modeling survival data with informative cluster size. Statistics in Medicine. 27, 543-555.

Yashin, A. I., and Iachine, I. A. (1995). Genetic analysis of durations: correlated frailty model applied to survival of Danish twins. Genetic Epidemiology. 12, 529-538.

Zhang, J. J., and Peng, Y. W. (2007). An alternative estimation method for the accelerated failure time frailty model. Computational Statistics \& Data Analysis. $51,4413-4423$.

Liang, K. and Zeger, S. (1986). Longitudinal data analysis using generalized linear models. Biometrika. 73, 13-22.

Zeng, D. G, and Lin, D. Y. (2007). Efficient estimation for the accelerated failure time model. Journal of the American Statistical Association. 102, 1387-1396.

Zheng, Y., and Heagerty, P. (2005). Partly conditional survival models for longitudinal data. Biometrics. 61, 379-391. 


\section{APPENDIX I}

\section{Technical Details}

Regularity conditions for the theorems.

(i) $n_{j} /\left(n_{1}+n_{2}\right) \rightarrow c_{j} \in(0,1)$, for $j=1,2$.

(ii) $\int \omega_{1}^{2}(t) \lambda_{C_{1}}(t) d t<\infty$ and $\int\left\{\omega_{2}^{2}(t)+\omega_{3}^{2}(t)\right\} \lambda_{C_{2}}(t) d t<\infty$.

(iii) $\int \frac{S_{2}^{2}\left(w_{1}\right)}{K_{1}^{2}\left(v_{1}-\right)} d \bar{n}_{1}\left(w_{1}, v_{1}\right)<\infty, \int \frac{F_{1}^{2}\left(w_{2}-\right)}{K_{2}^{2}\left(v_{2}-\right)} d \bar{n}_{2}\left(w_{2}, v_{2}\right)<\infty$,

and $\int \frac{d F_{1}\left(w_{1}\right)}{K_{2}^{2}\left(w_{1}+x_{2}-\right)} d \bar{n}_{3}\left(w_{2}, x_{2}\right)<\infty$

Proof of Theorem 1. Express

$$
\begin{gathered}
\sqrt{ } n\left(\widehat{U}_{1}-\theta\right)=\sqrt{ } n\left(U_{1}-\theta\right) \\
-\frac{\sqrt{ } n}{n_{1} n_{2}} \sum_{i_{1}, i_{2}} \frac{I\left(W_{i_{1}, 1}<W_{i_{2}, 2}\right) \delta_{i_{1}, 1} \delta_{i_{2}, 2}}{\widehat{K}_{1}\left(V_{i_{1}, 1}-\right)}\left\{\frac{\widehat{K}_{2}\left(V_{i_{2}, 2}-\right)-K_{2}\left(V_{i_{2}, 2}-\right)}{\widehat{K}_{2}\left(V_{i_{2}, 2}-\right) K_{2}\left(V_{i_{2}, 2}-\right)}\right\} \\
-\frac{\sqrt{ } n}{n_{1} n_{2}} \sum_{i_{1}, i_{2}} \frac{I\left(W_{i_{1}, 1}<W_{i_{2}, 2}\right) \delta_{i_{1}, 1} \delta_{i_{2}, 2}}{K_{2}\left(V_{i_{2}, 2}-\right)}\left\{\frac{\widehat{K}_{1}\left(V_{i_{1}, 1}-\right)-K_{1}\left(V_{i_{1}, 1}-\right)}{\widehat{K}_{1}\left(V_{i_{1}, 1}-\right) K_{1}\left(V_{i_{1}, 1}-\right)}\right\} .
\end{gathered}
$$

By an $L_{1}$ analysis of the difference as in Datta et al. (2010), we can replace $\widehat{K}_{j}, j=1,2$, by their in probability limits $K_{j}$ in the denominator of the last two terms provided we add an extra $o_{p}(1)$ term. Since

$$
\sqrt{ } n_{j}\left(\widehat{K}_{j}-K_{j}\right)=-\sqrt{ } n_{j} K_{j}\left(\widehat{\Lambda}_{j}^{c}-\Lambda_{j}^{c}\right)+o_{p}(1),
$$


by the delta method, where $\Lambda_{j}^{c}$ is the cumulative censoring hazard in group $j$ and $\widehat{\Lambda}_{j}^{c}$ is its Nelson-Aalen estimator,

$$
\begin{gathered}
\sqrt{ } n\left(\hat{U}_{1}-\theta\right)=\sqrt{ } n\left(U_{1}-\theta\right)+\frac{\sqrt{ } n}{n_{1} n_{2}} \sum_{i_{1}, i_{2}}\left[\frac{I\left(W_{i_{1}, 1}<W_{i_{2}, 2}\right) \delta_{i_{1}, 1} \delta_{i_{2}, 2}}{K_{1}\left(V_{i_{1}, 1}-\right) K_{2}\left(V_{i_{2}, 2}-\right)}\right. \\
\left.\times\left\{\widehat{\Lambda}_{1}^{c}\left(V_{i_{1}, 1}-\right)-\Lambda_{1}^{c}\left(V_{i_{1}, 1}-\right)+\widehat{\Lambda}_{2}^{c}\left(V_{i_{2}, 2}-\right)-\Lambda_{2}^{c}\left(V_{i_{2}, 2}-\right)\right\}\right]+o_{p}(1) ;
\end{gathered}
$$

the details can be worked out by an $L_{2}$ analysis of the error term in (A1). By $L_{2}$ projection calculations as in Hoeffding's decomposition (Hoeffding, 1948; Serfling, 1980) the above equals

$$
\begin{aligned}
& \frac{1}{\sqrt{ } n}\left[\frac{1}{c_{1}} \sum_{i_{1}=1}^{n_{1}}\left\{\frac{S_{2}\left(W_{i_{1}, 1}\right) \delta_{i_{1}, 1}}{K_{1}\left(V_{i_{1}, 1}-\right)}-\theta\right\}+\frac{1}{c_{2}} \sum_{i_{2}=1}^{n_{2}}\left\{\frac{F_{1}\left(W_{i_{2}, 2}-\right) \delta_{i_{2}, 2}}{K_{2}\left(V_{i_{2}, 2}-\right)}-\theta\right\}\right. \\
& +\frac{1}{c_{1}} \sum_{i_{1}=1}^{n_{1}} \frac{S_{2}\left(W_{i_{1}, 1}\right) \delta_{i_{1}, 1}}{K_{1}\left(V_{i_{1}, 1}-\right)}\left\{\widehat{\Lambda}_{1}^{c}\left(V_{i_{1}, 1}-\right)-\Lambda_{1}^{c}\left(V_{i_{1}, 1}-\right)\right\} \\
& \left.+\frac{1}{c_{2}} \sum_{i_{2}=1}^{n_{2}} \frac{F_{1}\left(W_{i_{2}, 2}-\right) \delta_{i_{2}, 2}}{K_{2}\left(V_{i_{2}, 2}-\right)}\left\{\widehat{\Lambda}_{2}^{c}\left(V_{i_{2}, 2}-\right)-\Lambda_{2}^{c}\left(V_{i_{2}, 2}-\right)\right\}\right]+o_{p}(1) .
\end{aligned}
$$

Using a martingale representation (Andersen et al., 1993, page 178) for $\widehat{\Lambda}_{j}^{c}(\cdot)-\Lambda_{j}(\cdot)$, we see that the above expression equals

$$
\begin{gathered}
\frac{1}{\sqrt{ } n}\left[\frac{1}{c_{1}} \sum_{i_{1}=1}^{n_{1}}\left\{\frac{S_{2}\left(W_{i_{1}, 1}\right) \delta_{i_{1}, 1}}{K_{1}\left(V_{i_{1}, 1}-\right)}-\theta\right\}+\frac{1}{c_{2}} \sum_{i_{2}=1}^{n_{2}}\left\{\frac{F_{1}\left(W_{i_{2}, 2}-\right) \delta_{i_{2}, 2}}{K_{2}\left(V_{i_{2}, 2}-\right)}-\theta\right\}\right. \\
+\frac{1}{c_{1} \sqrt{ } n_{1}} \sum_{i_{1}=1}^{n_{1}} \frac{S_{2}\left(W_{i_{1}, 1}\right) \delta_{i_{1}, 1}}{K_{1}\left(V_{i_{1}, 1}-\right)}\left\{\int_{0}^{V_{i_{1}, 1}-} \frac{d \bar{M}_{1}^{c}(s)}{y_{1}(s)}\right\} \\
\left.+\frac{1}{c_{2} \sqrt{ } n_{2}} \sum_{i_{2}=1}^{n_{2}} \frac{F_{1}\left(W_{i_{2}, 2}-\right) \delta_{i_{2}, 2}}{K_{2}\left(V_{i_{2}, 2}-\right)}\left\{\int_{0}^{V_{i_{2}, 2}-} \frac{d \bar{M}_{2}^{c}(s)}{y_{2}(s)}\right\}\right]+o_{p}(1) ;
\end{gathered}
$$

here $M_{i, j}^{c}(t)=N_{i, j}^{c}(t)-\int_{0}^{t} Y_{i, j}(u) d \Lambda_{j}^{c}(u), N_{i, j}^{c}(t)=I\left(V_{i, j} \leq t, \delta_{i, j}=0\right), Y_{i, j}(t)=$ $I\left(V_{i, j} \geq t\right), \quad \bar{M}_{j}=n_{j}^{-1 / 2} \sum_{i=1}^{n_{j}} M_{i, j}^{c} \quad$ and $\quad y_{j}(t)=E Y_{i, j}(t), j=1,2 . \quad$ From $\quad$ the 
asymptotically linear representation of a $U$-statistic (Serfling, 1980), the second term in the RHS of (A2) equals

$$
\frac{\sqrt{ } n_{1}}{c_{1}} \int \frac{S_{2}\left(w_{1}\right)}{K_{1}\left(v_{1}-\right)}\left\{\int_{0}^{v_{1}-} \frac{d \bar{M}_{1}^{c}(s)}{y_{1}(s)}\right\} d \bar{n}_{1}\left(w_{1}, v_{1}\right)+o_{p}\left(\sqrt{ } n_{1}\right)
$$

which further equals, by Fubini's theorem,

$$
\begin{gathered}
\frac{1}{c_{1}} \sum_{i_{1}=1}^{n_{1}} \int_{0}^{\infty}\left\{\frac{1}{y_{1}(s)} \int I\left(v_{1}>s\right) \frac{S_{2}\left(w_{1}\right)}{K_{1}\left(v_{1}-\right)} d \bar{n}_{1}\left(w_{1}, v_{1}\right)\right\} d M_{i_{1}, 1}^{c}(s)+o_{p}\left(\sqrt{ } n_{1}\right), \\
=\frac{1}{c_{1}} \sum_{i_{1}=1}^{n_{1}} \int_{0}^{\infty} \omega_{1}(s) d M_{i_{1}, 1}^{c}(s)+o_{p}\left(\sqrt{ } n_{1}\right),
\end{gathered}
$$

where $\omega_{1}$ is given in (2). The third term can be handled the same way leading to the following linearization

$$
\begin{gathered}
\sqrt{ } n\left(\widehat{U}_{1}-\theta\right)=\frac{1}{\sqrt{ } n}\left[\frac{1}{c_{1}} \sum_{i_{1}=1}^{n_{1}}\left\{\frac{S_{2}\left(W_{i_{1}, 1}\right) \delta_{i_{1}, 1}}{K_{1}\left(V_{i_{1}, 1}-\right)}-\theta+\int_{0}^{\infty} \omega_{1}(s) d M_{i_{1}, 1}^{c}(s)\right\}\right. \\
\left.+\frac{1}{c_{2}} \sum_{i_{2}=1}^{n_{2}}\left\{\frac{F_{1}\left(W_{i_{2}, 2}-\right) \delta_{i_{2}, 2}}{K_{2}\left(V_{i_{2}, 2}-\right)}-\theta+\int_{0}^{\infty} \omega_{2}(s) d M_{i_{2}, 2}^{c}(s)\right\}\right]+o_{p}(1)
\end{gathered}
$$

where $\omega_{2}$ is given by (3). Therefore, we have, as $n \rightarrow \infty$,

$$
\sqrt{ } n\left(\widehat{U}_{1}-\theta\right) \stackrel{d}{\rightarrow} N\left(0, \sigma_{1}^{2}\right)
$$

where $\sigma_{1}^{2}$ is as in the statement of Theorem 1 . This proves the first assertation of Theorem 1. The linearlization for $\widehat{U}_{2}$ can be carried out in a similar fashion.

Estimation of variance. We estimate the asymptotic variance by the empirical variance of the linear approximation (A.6). Note that

$$
\int \omega_{j}(s) d \widehat{M}_{i, j}^{c}(s)=\omega_{j}\left(V_{i, j}\right) \bar{\delta}_{i, j}-\int \frac{\omega_{j}(s) I\left(V_{i, j} \geq s\right)}{Y_{j}(s)} d N_{j}^{c}(s),
$$

where $\widehat{M}_{i, j}^{c}(t)=N_{i, j}^{c}(t)-\int_{0}^{t} Y_{i, j}(u) d \widehat{\Lambda}_{j}^{c}(u), \widehat{\Lambda}_{j}^{c}$ being the Nelson-Aalen estimator of $\Lambda_{j}^{c}$, 


$$
\begin{gathered}
=\omega_{j}\left(V_{i, j}\right) \bar{\delta}_{i, j}-\int_{0}^{\infty} \frac{\omega_{j}(s) I\left(V_{i, j} \geq s\right)}{Y_{j}(s)} d\left\{\sum_{i_{1}=1}^{n_{j}} N_{i_{1}, j}^{c}(s)\right\} \\
=\omega_{j}\left(V_{i, j}\right) \bar{\delta}_{i, j}-\sum_{i_{1}=1}^{n_{j}} \frac{\omega_{j}\left(V_{i_{1}, j}\right) I\left(V_{i, j} \geq V_{i_{1}, j}\right) \bar{\delta}_{i_{1}, j}}{Y_{j}\left(V_{i_{1}, j}\right)}
\end{gathered}
$$

This justifies the choice of the summands $S_{1,2}$. The other parts can be obtained in a similar fashion. Its consistency can be established using laws of large numbers for $U$ statistics.

Proof of Theorem 2. Asymptotic linearlization of the test statistic is obtained as a linear combination of the linear approximations of the statistics $\widehat{U}(1,2)$ and $\widehat{U}(2,1)$ as obtained under Theorem 1. 


\section{APPENDIX II}

\section{Additional Simulation Results}

Table A1

Simulation results for the cluster-weighted AFT model when the cluster size is only related to the cluster effect $($ Beta $1=0.8$ Beta $2=1$, Beta3 $=-0.75$, Beta4 $=-1.5$, alpha $=0.25$,

$$
\mathrm{rl}=1.5, \mathrm{r} 2=3, \mathrm{z} 3=\mathrm{N}(1,0.5) \text {, error }=0.05) \text {. }
$$

\begin{tabular}{|c|c|c|c|c|}
\hline \multirow[t]{2}{*}{ Number of cluster } & & \multicolumn{3}{|c|}{ Censoring rate } \\
\hline & & 0.25 & 0.5 & 0.75 \\
\hline \multirow[t]{12}{*}{30} & $\operatorname{Bias}\left(\widehat{\beta}_{1}\right)$ & 0.059 & 0.043 & -0.016 \\
\hline & $\operatorname{e.sd}\left(\widehat{\beta}_{1}\right)$ & 0.139 & 0.165 & 0.256 \\
\hline & $\operatorname{sd}\left(\widehat{\beta}_{1}\right)$ & 0.113 & 0.130 & 0.219 \\
\hline & $\operatorname{Bias}\left(\hat{\beta}_{2}\right)$ & -0.022 & -0.040 & -0.009 \\
\hline & $\operatorname{e.sd}\left(\widehat{\beta}_{2}\right)$ & 0.219 & 0.282 & 0.382 \\
\hline & $\operatorname{sd}\left(\widehat{\beta}_{2}\right)$ & 0.181 & 0.212 & 0.304 \\
\hline & $\operatorname{Bias}\left(\hat{\beta}_{3}\right)$ & -0.011 & 0.007 & 0.036 \\
\hline & $\operatorname{e.sd}\left(\widehat{\beta}_{3}\right)$ & 0.106 & 0.122 & 0.186 \\
\hline & $\operatorname{sd}\left(\widehat{\beta}_{3}\right)$ & 0.084 & 0.100 & 0.162 \\
\hline & $\operatorname{Bias}\left(\widehat{\beta}_{4}\right)$ & 0.001 & 0.009 & -0.004 \\
\hline & $\operatorname{e.sd}\left(\widehat{\beta}_{4}\right)$ & 0.169 & 0.209 & 0.274 \\
\hline & $\operatorname{sd}\left(\widehat{\beta}_{4}\right)$ & 0.134 & 0.158 & 0.220 \\
\hline \multirow[t]{12}{*}{200} & $\operatorname{Bias}\left(\widehat{\beta}_{1}\right)$ & 0.109 & 0.099 & 0.026 \\
\hline & $\operatorname{e.sd}\left(\widehat{\beta}_{1}\right)$ & 0.077 & 0.088 & 0.123 \\
\hline & $\operatorname{sd}\left(\widehat{\beta}_{1}\right)$ & 0.059 & 0.071 & 0.108 \\
\hline & $\operatorname{Bias}\left(\widehat{\beta}_{2}\right)$ & -0.039 & -0.065 & -0.044 \\
\hline & $\operatorname{e.sd}\left(\widehat{\beta}_{2}\right)$ & 0.114 & 0.141 & 0.204 \\
\hline & $\operatorname{sd}\left(\widehat{\beta}_{2}\right)$ & 0.096 & 0.115 & 0.160 \\
\hline & $\operatorname{Bias}\left(\widehat{\beta}_{3}\right)$ & -0.048 & -0.029 & 0.019 \\
\hline & $\operatorname{e} . \operatorname{sd}\left(\widehat{\beta}_{3}\right)$ & 0.055 & 0.064 & 0.090 \\
\hline & $\operatorname{sd}\left(\widehat{\beta}_{3}\right)$ & 0.043 & 0.052 & 0.079 \\
\hline & $\operatorname{Bias}\left(\widehat{\beta}_{4}\right)$ & 0.008 & 0.016 & 0.003 \\
\hline & $\operatorname{e.sd}\left(\widehat{\beta}_{4}\right)$ & 0.084 & 0.102 & 0.146 \\
\hline & $\operatorname{sd}\left(\widehat{\beta}_{4}\right)$ & 0.069 & 0.083 & 0.113 \\
\hline
\end{tabular}




\section{Table A2}

Simulation results for the non-cluster-weighted AFT model when the cluster size is only related to the cluster effect $($ Beta $1=0.8$ Beta2 $=1$, Beta $3=-0.75$, Beta4 $=-1.5$, alpha $=0.25$, $\mathrm{r} 1=1.5, \mathrm{r} 2=3, \mathrm{z} 3=\mathrm{N}(1,0.5)$, error $=0.05)$.

\begin{tabular}{llrrr}
\hline Number of cluster & & \multicolumn{3}{c}{ Censoring rate } \\
& & 0.25 & 0.5 & 0.75 \\
\hline 30 & $\operatorname{Bias}\left(\widehat{\beta}_{1}\right)$ & 0.183 & 0.144 & 0.051 \\
& $\operatorname{e.sd}\left(\widehat{\beta}_{1}\right)$ & 0.140 & 0.161 & 0.252 \\
& $\operatorname{sd}\left(\widehat{\beta}_{1}\right)$ & 0.085 & 0.111 & 0.173 \\
& $\operatorname{Bias}\left(\widehat{\beta}_{2}\right)$ & -0.024 & -0.032 & -0.004 \\
& $\operatorname{e.sd}\left(\widehat{\beta}_{2}\right)$ & 0.218 & 0.273 & 0.362 \\
& $\operatorname{sd}\left(\widehat{\beta}_{2}\right)$ & 0.142 & 0.177 & 0.247 \\
& $\operatorname{Bias}\left(\widehat{\beta}_{3}\right)$ & 0.002 & 0.033 & 0.075 \\
& $\operatorname{e.sd}\left(\widehat{\beta}_{3}\right)$ & 0.090 & 0.109 & 0.177 \\
& $\operatorname{sd}\left(\widehat{\beta}_{3}\right)$ & 0.070 & 0.086 & 0.129 \\
& $\operatorname{Bias}\left(\widehat{\beta}_{4}\right)$ & 0.001 & 0.003 & -0.001 \\
& $\operatorname{e.sd}\left(\widehat{\beta}_{4}\right)$ & 0.145 & 0.186 & 0.248 \\
& $\operatorname{sd}\left(\widehat{\beta}_{4}\right)$ & 0.114 & 0.134 & 0.178 \\
& & & \\
& $\operatorname{Bias}\left(\widehat{\beta}_{1}\right)$ & 0.277 & 0.243 & 0.138 \\
& $\operatorname{e.sd}\left(\widehat{\beta}_{1}\right)$ & 0.099 & 0.102 & 0.137 \\
& $\operatorname{sd}\left(\widehat{\beta}_{1}\right)$ & 0.056 & 0.073 & 0.113 \\
& $\operatorname{Bias}\left(\widehat{\beta}_{2}\right)$ & -0.040 & -0.056 & -0.023 \\
& $\operatorname{e.sd}\left(\widehat{\beta}_{2}\right)$ & 0.155 & 0.165 & 0.230 \\
& $\operatorname{sd}\left(\widehat{\beta}_{2}\right)$ & 0.097 & 0.123 & 0.170 \\
& $\operatorname{Bias}\left(\widehat{\beta}_{3}\right)$ & -0.042 & -0.005 & 0.058 \\
& $\operatorname{e.sd}\left(\widehat{\beta}_{3}\right)$ & 0.062 & 0.074 & 0.101 \\
& $\operatorname{sd}\left(\widehat{\beta}_{3}\right)$ & 0.044 & 0.055 & 0.082 \\
& $\operatorname{Bias}\left(\widehat{\beta}_{4}\right)$ & 0.002 & -0.001 & -0.016 \\
& $\operatorname{e.sd}\left(\widehat{\beta}_{4}\right)$ & 0.099 & 0.117 & 0.162 \\
$\operatorname{sd}\left(\widehat{\beta}_{4}\right)$ & 0.074 & 0.089 & 0.118 \\
\hline & & &
\end{tabular}




\section{Table A3}

Simulation results for the cluster-weighted AFT model when the cluster size is only related to the cluster effect (Beta1 $=1.5$ Beta $2=2$, Beta $3=-1$, Beta $4=-1.5$, alpha $=0.15$, $\mathrm{r} 1=1, \mathrm{r} 2=4, \mathrm{z} 3=\mathrm{N}(1,0.5)$, error $=0.05)$.

\begin{tabular}{|c|c|c|c|c|}
\hline \multirow[t]{2}{*}{ Number of cluster } & & \multicolumn{3}{|c|}{ Censoring rate } \\
\hline & & 0.25 & 0.5 & 0.75 \\
\hline \multirow[t]{12}{*}{30} & $\operatorname{Bias}\left(\widehat{\beta}_{1}\right)$ & 0.015 & 0.017 & -0.028 \\
\hline & e.sd $\left(\widehat{\beta}_{1}\right)$ & 0.093 & 0.143 & 0.221 \\
\hline & $\operatorname{sd}\left(\widehat{\beta}_{1}\right)$ & 0.074 & 0.104 & 0.162 \\
\hline & $\operatorname{Bias}\left(\widehat{\beta}_{2}\right)$ & -0.007 & -0.053 & -0.108 \\
\hline & e.sd $\left(\widehat{\beta}_{2}\right)$ & 0.199 & 0.321 & 0.521 \\
\hline & $\operatorname{sd}\left(\widehat{\beta}_{2}\right)$ & 0.156 & 0.216 & 0.340 \\
\hline & $\operatorname{Bias}\left(\widehat{\beta}_{3}\right)$ & 0.003 & 0.003 & 0.031 \\
\hline & e.sd $\left(\widehat{\beta}_{3}\right)$ & 0.074 & 0.108 & 0.249 \\
\hline & $\operatorname{sd}\left(\widehat{\beta}_{3}\right)$ & 0.060 & 0.080 & 0.113 \\
\hline & $\operatorname{Bias}\left(\widehat{\beta}_{4}\right)$ & 0.006 & 0.039 & 0.077 \\
\hline & $\operatorname{e} . \operatorname{sd}\left(\widehat{\beta}_{4}\right)$ & 0.149 & 0.234 & 0.347 \\
\hline & $\operatorname{sd}\left(\widehat{\beta}_{4}\right)$ & 0.119 & 0.158 & 0.229 \\
\hline \multirow[t]{12}{*}{200} & $\operatorname{Bias}\left(\widehat{\beta}_{1}\right)$ & 0.036 & 0.041 & 0.009 \\
\hline & e.sd $\left(\widehat{\beta}_{1}\right)$ & 0.048 & 0.077 & 0.119 \\
\hline & $\operatorname{sd}\left(\widehat{\beta}_{1}\right)$ & 0.040 & 0.061 & 0.088 \\
\hline & $\operatorname{Bias}\left(\widehat{\beta}_{2}\right)$ & 0.024 & 0.003 & -0.052 \\
\hline & e.sd $\left(\widehat{\beta}_{2}\right)$ & 0.113 & 0.178 & 0.299 \\
\hline & $\operatorname{sd}\left(\widehat{\beta}_{2}\right)$ & 0.087 & 0.126 & 0.179 \\
\hline & $\operatorname{Bias}\left(\widehat{\beta}_{3}\right)$ & -0.013 & -0.011 & 0.014 \\
\hline & e.sd $\left(\widehat{\beta}_{3}\right)$ & 0.038 & 0.058 & 0.083 \\
\hline & $\operatorname{sd}\left(\widehat{\beta}_{3}\right)$ & 0.031 & 0.045 & 0.061 \\
\hline & $\operatorname{Bias}\left(\widehat{\beta}_{4}\right)$ & -0.015 & 0.001 & 0.039 \\
\hline & e.sd $\left(\widehat{\beta}_{4}\right)$ & 0.085 & 0.129 & 0.206 \\
\hline & $\operatorname{sd}\left(\widehat{\beta}_{4}\right)$ & 0.065 & 0.089 & 0.119 \\
\hline
\end{tabular}




\section{Table A4}

Simulation results for the n0n-cluster-weighted AFT model when the cluster size is only related to the cluster effect $($ Beta $1=1.5$ Beta2 $=2$, Beta $3=-1$, Beta4 $=-1.5$, alpha $=0.15$, $\mathrm{r} 1=1, \mathrm{r} 2=4, \mathrm{z} 3=\mathrm{N}(1,0.5)$, error $=0.05)$

\begin{tabular}{|c|c|c|c|c|}
\hline \multirow[t]{2}{*}{ Number of cluster } & & \multicolumn{3}{|c|}{ Censoring rate } \\
\hline & & 0.25 & 0.5 & 0.75 \\
\hline \multirow[t]{12}{*}{30} & $\operatorname{Bias}\left(\widehat{\beta}_{1}\right)$ & 0.069 & 0.059 & -0.003 \\
\hline & e.sd $\left(\widehat{\beta}_{1}\right)$ & 0.091 & 0.134 & 0.219 \\
\hline & $\operatorname{sd}\left(\widehat{\beta}_{1}\right)$ & 0.062 & 0.095 & 0.146 \\
\hline & $\operatorname{Bias}\left(\widehat{\beta}_{2}\right)$ & -0.022 & -0.072 & -0.136 \\
\hline & $\operatorname{e.sd}\left(\widehat{\beta}_{2}\right)$ & 0.190 & 0.309 & 0.526 \\
\hline & $\operatorname{sd}\left(\widehat{\beta}_{2}\right)$ & 0.141 & 0.201 & 0.266 \\
\hline & $\operatorname{Bias}\left(\widehat{\beta}_{3}\right)$ & 0.006 & 0.015 & 0.050 \\
\hline & $\operatorname{e.sd}\left(\widehat{\beta}_{3}\right)$ & 0.065 & 0.099 & 0.144 \\
\hline & $\operatorname{sd}\left(\widehat{\beta}_{3}\right)$ & 0.054 & 0.074 & 0.103 \\
\hline & $\operatorname{Bias}\left(\widehat{\beta}_{4}\right)$ & 0.017 & 0.053 & 0.096 \\
\hline & $\operatorname{e.sd}\left(\widehat{\beta}_{4}\right)$ & 0.134 & 0.222 & 0.347 \\
\hline & $\operatorname{sd}\left(\widehat{\beta}_{4}\right)$ & 0.110 & 0.148 & 0.183 \\
\hline \multirow[t]{12}{*}{200} & $\operatorname{Bias}\left(\widehat{\beta}_{1}\right)$ & 0.105 & 0.104 & 0.054 \\
\hline & $\operatorname{e.sd}\left(\widehat{\beta}_{1}\right)$ & 0.056 & 0.083 & 0.123 \\
\hline & $\operatorname{sd}\left(\widehat{\beta}_{1}\right)$ & 0.037 & 0.062 & 0.089 \\
\hline & $\operatorname{Bias}\left(\widehat{\beta}_{2}\right)$ & 0.021 & -0.015 & -0.084 \\
\hline & e.sd $\left(\widehat{\beta}_{2}\right)$ & 0.122 & 0.190 & 0.320 \\
\hline & $\operatorname{sd}\left(\widehat{\beta}_{2}\right)$ & 0.089 & 0.137 & 0.192 \\
\hline & $\operatorname{Bias}\left(\widehat{\beta}_{3}\right)$ & -0.013 & -0.005 & 0.031 \\
\hline & $\operatorname{e.sd}\left(\widehat{\beta}_{3}\right)$ & 0.040 & 0.062 & 0.086 \\
\hline & $\operatorname{sd}\left(\widehat{\beta}_{3}\right)$ & 0.031 & 0.047 & 0.062 \\
\hline & $\operatorname{Bias}\left(\widehat{\beta}_{4}\right)$ & -0.013 & 0.013 & 0.061 \\
\hline & e.sd $\left(\widehat{\beta}_{4}\right)$ & 0.087 & 0.137 & 0.223 \\
\hline & $\operatorname{sd}\left(\widehat{\beta}_{4}\right)$ & 0.068 & 0.099 & 0.132 \\
\hline
\end{tabular}




\section{Table A5}

Simulation results for the cluster-weighted AFT model when the cluster size is not informative $($ Beta $1=0.3$ Beta $2=0.4$, Beta $3=-0.2$, Beta $4=-0.3$, alpha $=0.25$, pai $=0.15$,

$$
\mathrm{rl}=1.5, \mathrm{r} 2=3, \mathrm{z} 3=\mathrm{N}(1,0.15) \text {, error }=0.05) \text {. }
$$

\begin{tabular}{|c|c|c|c|c|}
\hline \multirow[t]{2}{*}{ Number of cluster } & & \multicolumn{3}{|c|}{ Censoring rate } \\
\hline & & 0.25 & 0.5 & 0.75 \\
\hline \multirow[t]{12}{*}{30} & $\operatorname{Bias}\left(\widehat{\beta}_{1}\right)$ & 0.030 & 0.049 & 0.015 \\
\hline & e.sd $\left(\widehat{\beta}_{1}\right)$ & 0.248 & 0.308 & 0.435 \\
\hline & $\operatorname{sd}\left(\widehat{\beta}_{1}\right)$ & 0.227 & 0.263 & 0.336 \\
\hline & $\operatorname{Bias}\left(\widehat{\beta}_{2}\right)$ & -0.054 & -0.080 & -0.109 \\
\hline & $\operatorname{e.sd}\left(\widehat{\beta}_{2}\right)$ & 0.396 & 0.544 & 0.749 \\
\hline & $\operatorname{sd}\left(\widehat{\beta}_{2}\right)$ & 0.346 & 0.419 & 0.565 \\
\hline & $\operatorname{Bias}\left(\widehat{\beta}_{3}\right)$ & -0.006 & -0.018 & 0.012 \\
\hline & $\operatorname{e.sd}\left(\widehat{\beta}_{3}\right)$ & 0.239 & 0.294 & 0.427 \\
\hline & $\operatorname{sd}\left(\widehat{\beta}_{3}\right)$ & 0.217 & 0.255 & 0.328 \\
\hline & $\operatorname{Bias}\left(\widehat{\beta}_{4}\right)$ & 0.039 & 0.053 & 0.066 \\
\hline & $\operatorname{e.sd}\left(\widehat{\beta}_{4}\right)$ & 0.382 & 0.531 & 0.723 \\
\hline & $\operatorname{sd}\left(\widehat{\beta}_{4}\right)$ & 0.330 & 0.405 & 0.546 \\
\hline \multirow[t]{12}{*}{200} & $\operatorname{Bias}\left(\widehat{\beta}_{1}\right)$ & 0.019 & 0.043 & 0.063 \\
\hline & $\operatorname{e.sd}\left(\widehat{\beta}_{1}\right)$ & 0.106 & 0.147 & 0.222 \\
\hline & $\operatorname{sd}\left(\widehat{\beta}_{1}\right)$ & 0.102 & 0.134 & 0.197 \\
\hline & $\operatorname{Bias}\left(\widehat{\beta}_{2}\right)$ & -0.035 & -0.050 & -0.075 \\
\hline & $\operatorname{e.sd}\left(\widehat{\beta}_{2}\right)$ & 0.168 & 0.235 & 0.364 \\
\hline & $\operatorname{sd}\left(\widehat{\beta}_{2}\right)$ & 0.157 & 0.211 & 0.318 \\
\hline & $\operatorname{Bias}\left(\widehat{\beta}_{3}\right)$ & 0.006 & 0.002 & -0.008 \\
\hline & $\operatorname{e.sd}\left(\widehat{\beta}_{3}\right)$ & 0.102 & 0.141 & 0.215 \\
\hline & $\operatorname{sd}\left(\widehat{\beta}_{3}\right)$ & 0.097 & 0.130 & 0.192 \\
\hline & $\operatorname{Bias}\left(\widehat{\beta}_{4}\right)$ & 0.021 & 0.022 & 0.037 \\
\hline & e.sd $\left(\widehat{\beta}_{4}\right)$ & 0.160 & 0.226 & 0.357 \\
\hline & $\operatorname{sd}\left(\widehat{\beta}_{4}\right)$ & 0.149 & 0.204 & 0.307 \\
\hline
\end{tabular}




\section{Table A6}

Simulation results for the non-cluster-weighted AFT model when the cluster size is not

$$
\begin{gathered}
\text { informative }(\text { Beta } 1=0.3 \text { Beta2 }=0.4, \text { Beta } 3=-0.2, \text { Beta } 4=-0.3 \text {, alpha }=0.25, \text { pai }=0.15, \\
r 1=1.5, \mathrm{r} 2=3, \mathrm{z} 3=\mathrm{N}(1,0.15), \text { error }=0.05) .
\end{gathered}
$$

\begin{tabular}{|c|c|c|c|c|}
\hline \multirow[t]{2}{*}{ Number of cluster } & & \multicolumn{3}{|c|}{ Censoring rate } \\
\hline & & 0.25 & 0.5 & 0.75 \\
\hline \multirow[t]{12}{*}{30} & $\operatorname{Bias}\left(\widehat{\beta}_{1}\right)$ & 0.028 & 0.048 & 0.232 \\
\hline & e.sd $\left(\widehat{\beta}_{1}\right)$ & 0.217 & 0.263 & 0.386 \\
\hline & $\operatorname{sd}\left(\widehat{\beta}_{1}\right)$ & 0.203 & 0.239 & 0.319 \\
\hline & $\operatorname{Bias}\left(\widehat{\beta}_{2}\right)$ & -0.051 & -0.074 & -0.108 \\
\hline & e.sd $\left(\widehat{\beta}_{2}\right)$ & 0.349 & 0.481 & 0.694 \\
\hline & $\operatorname{sd}\left(\widehat{\beta}_{2}\right)$ & 0.313 & 0.398 & 0.549 \\
\hline & $\operatorname{Bias}\left(\widehat{\beta}_{3}\right)$ & -0.003 & -0.016 & 0.006 \\
\hline & $\operatorname{e.sd}\left(\widehat{\beta}_{3}\right)$ & 0.202 & 0.247 & 0.377 \\
\hline & $\operatorname{sd}\left(\widehat{\beta}_{3}\right)$ & 0.199 & 0.236 & 0.314 \\
\hline & $\operatorname{Bias}\left(\widehat{\beta}_{4}\right)$ & 0.036 & 0.049 & 0.066 \\
\hline & $\operatorname{e.sd}\left(\widehat{\beta}_{4}\right)$ & 0.331 & 0.468 & 0.672 \\
\hline & $\operatorname{sd}\left(\widehat{\beta}_{4}\right)$ & 0.306 & 0.389 & 0.535 \\
\hline \multirow[t]{12}{*}{200} & $\operatorname{Bias}\left(\widehat{\beta}_{1}\right)$ & 0.019 & 0.042 & 0.062 \\
\hline & e.sd $\left(\widehat{\beta}_{1}\right)$ & 0.090 & 0.123 & 0.195 \\
\hline & $\operatorname{sd}\left(\widehat{\beta}_{1}\right)$ & 0.085 & 0.113 & 0.179 \\
\hline & $\operatorname{Bias}\left(\widehat{\beta}_{2}\right)$ & -0.033 & -0.045 & -0.073 \\
\hline & $\operatorname{e.sd}\left(\widehat{\beta}_{2}\right)$ & 0.142 & 0.202 & 0.319 \\
\hline & $\operatorname{sd}\left(\widehat{\beta}_{2}\right)$ & 0.131 & 0.186 & 0.296 \\
\hline & $\operatorname{Bias}\left(\widehat{\beta}_{3}\right)$ & 0.005 & 0.002 & -0.007 \\
\hline & $\operatorname{e.sd}\left(\widehat{\beta}_{3}\right)$ & 0.084 & 0.115 & 0.186 \\
\hline & $\operatorname{sd}\left(\widehat{\beta}_{3}\right)$ & 0.083 & 0.111 & 0.175 \\
\hline & $\operatorname{Bias}\left(\widehat{\beta}_{4}\right)$ & 0.019 & 0.017 & 0.036 \\
\hline & $\operatorname{e.sd}\left(\widehat{\beta}_{4}\right)$ & 0.133 & 0.191 & 0.312 \\
\hline & $\operatorname{sd}\left(\widehat{\beta}_{4}\right)$ & 0.128 & 0.180 & 0.287 \\
\hline
\end{tabular}



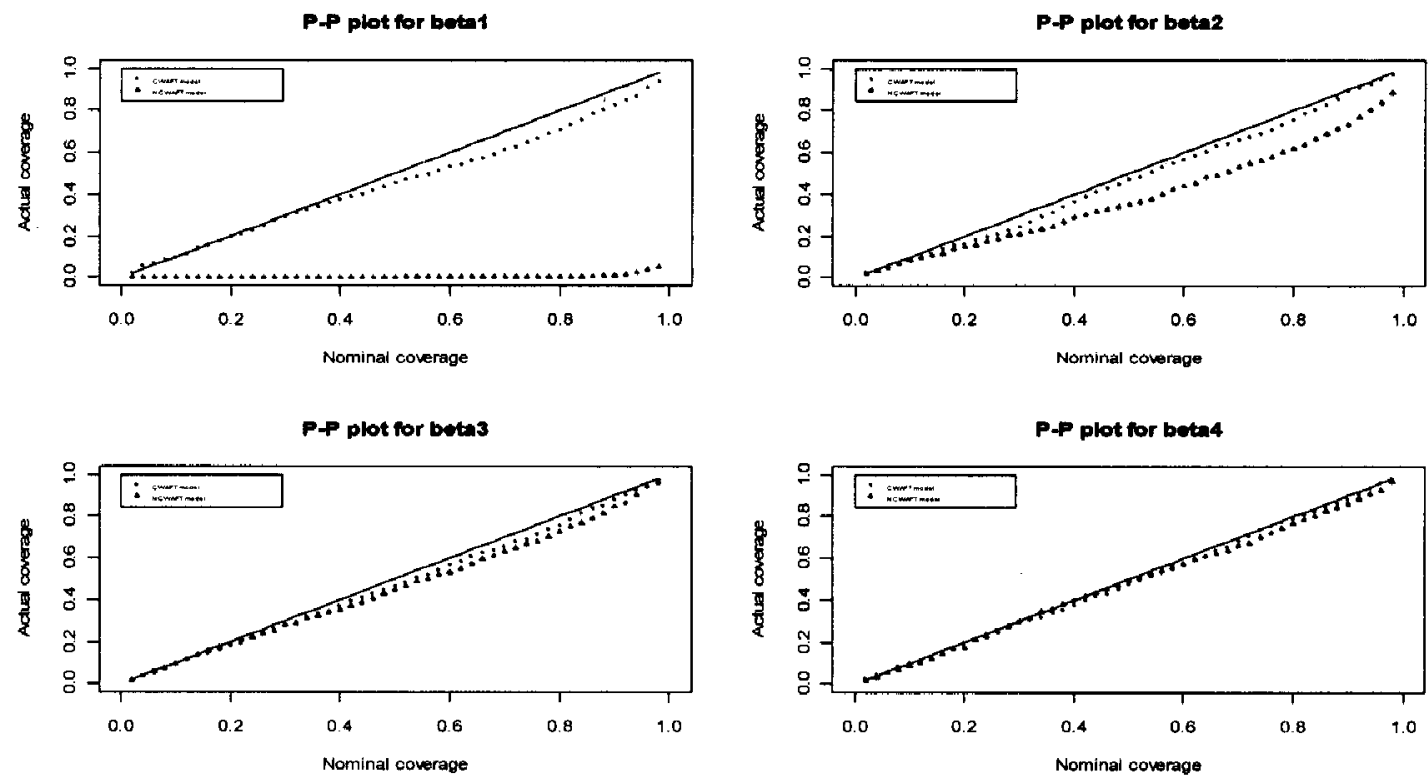

Figure A1. Probability-Probability plots for parameters for two competing methods when the cluster size is only related to the cluster effect using the same parameters in Table A1 -A2.
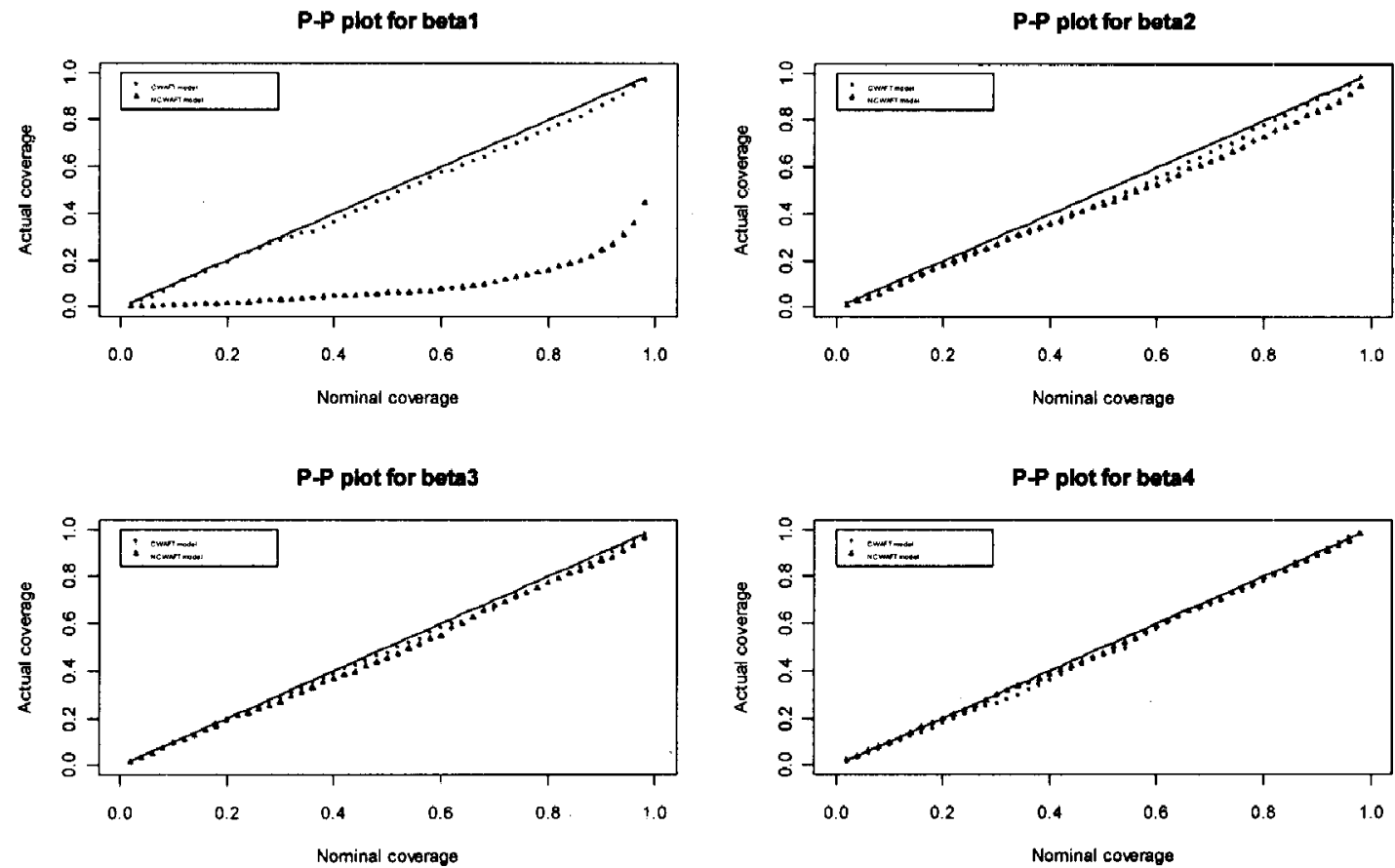

Figure A1. Probability-Probability plots for parameters for two competing methods when the cluster size is only related to the cluster effect using the same parameters in Table A3-A4. 

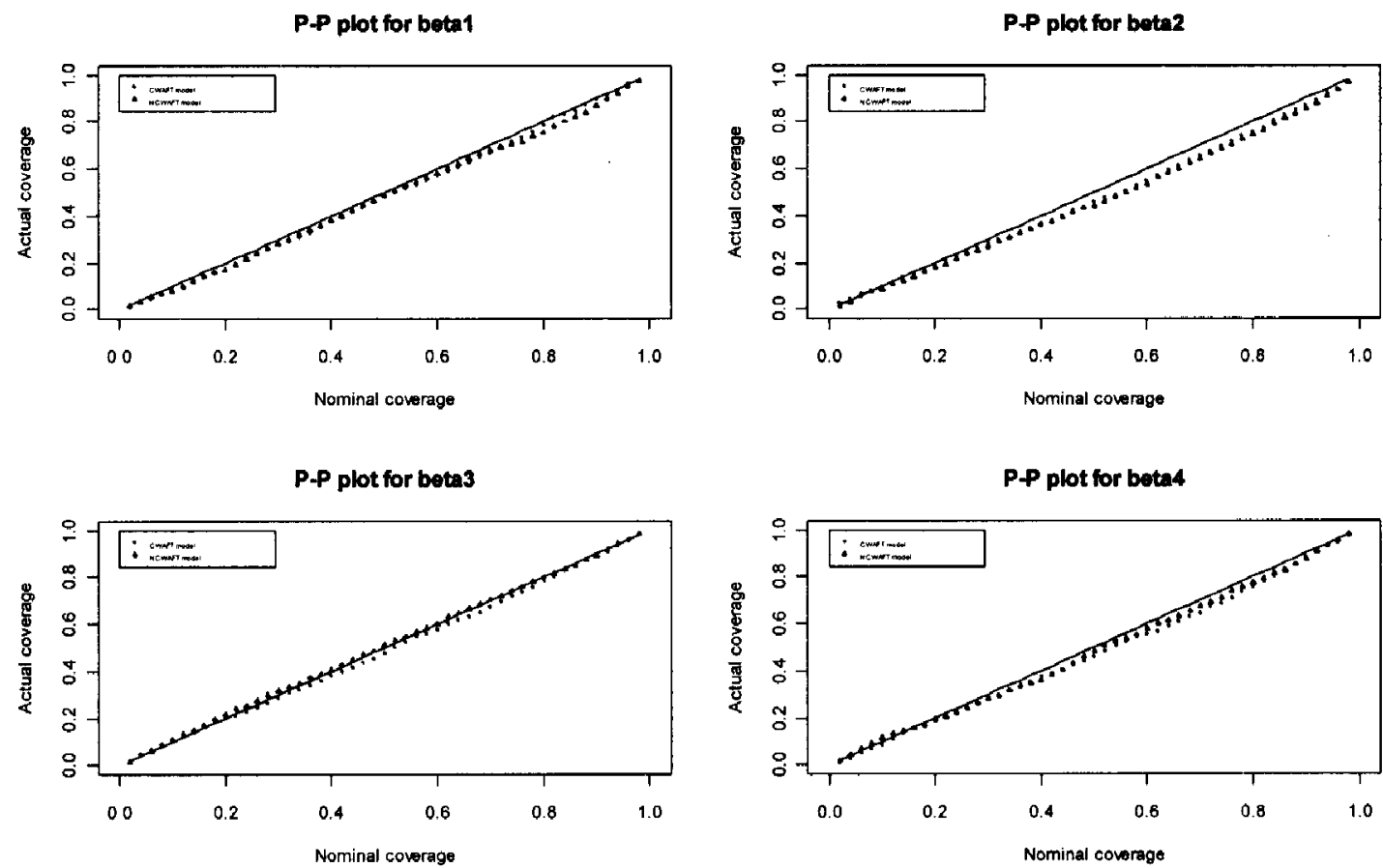

Figure Al Probability-Probability plots for parameters for two competing methods when the cluster size is not informative using the same parameters in Table A5 -A6. 
Table A7

Simulation results for $U$-statistics when two groups have same censoring rates but different group sizes.

\begin{tabular}{llrrr}
\hline Group size & & \multicolumn{3}{c}{ Censoring rate } \\
& & 0.25 & \multicolumn{1}{c}{0.5} & 0.75 \\
\hline$n_{1}=25$ & $\operatorname{Bias}\left(\widehat{U}_{1}\right)$ & -0.009 & -0.025 & -0.069 \\
$n_{2}=50$ & $\operatorname{Bias}\left(\widehat{U}_{2}\right)$ & -0.012 & -0.030 & -0.068 \\
& $\operatorname{e.se}\left(\widehat{U}_{1}\right)$ & 0.087 & 0.113 & 0.171 \\
& $\operatorname{e.se}\left(\widehat{U}_{2}\right)$ & 0.084 & 0.105 & 0.147 \\
& $\operatorname{se}\left(\widehat{U}_{1}\right)$ & 0.084 & 0.107 & 0.149 \\
& $\operatorname{se}\left(\widehat{U}_{2}\right)$ & 0.082 & 0.102 & 0.138 \\
\hline
\end{tabular}

Table A8

Simulation results for $U$-statistics when two groups have same censoring rates but different group sizes.

\begin{tabular}{llrrr}
\hline Group size & & \multicolumn{3}{c}{ Censoring rate } \\
& & 0.25 & \multicolumn{1}{c}{0.5} & 0.75 \\
\hline$n_{1}=100$ & $\operatorname{Bias}\left(\widehat{U}_{1}\right)$ & -0.008 & -0.022 & -0.058 \\
$n_{2}=50$ & $\operatorname{Bias}\left(\widehat{U}_{2}\right)$ & -0.012 & -0.027 & -0.062 \\
& $\operatorname{e.se}\left(\widehat{U}_{1}\right)$ & 0.060 & 0.082 & 0.131 \\
& $\operatorname{e.se}\left(\widehat{U}_{2}\right)$ & 0.057 & 0.071 & 0.104 \\
& $\operatorname{se}\left(\widehat{U}_{1}\right)$ & 0.060 & 0.078 & 0.111 \\
& $\operatorname{se}\left(\widehat{U}_{2}\right)$ & 0.056 & 0.068 & 0.098 \\
\hline
\end{tabular}




\section{APPENDIX III}

\section{Key R Programs for Correlated Survival Data with Potentially Informative Cluster Size}

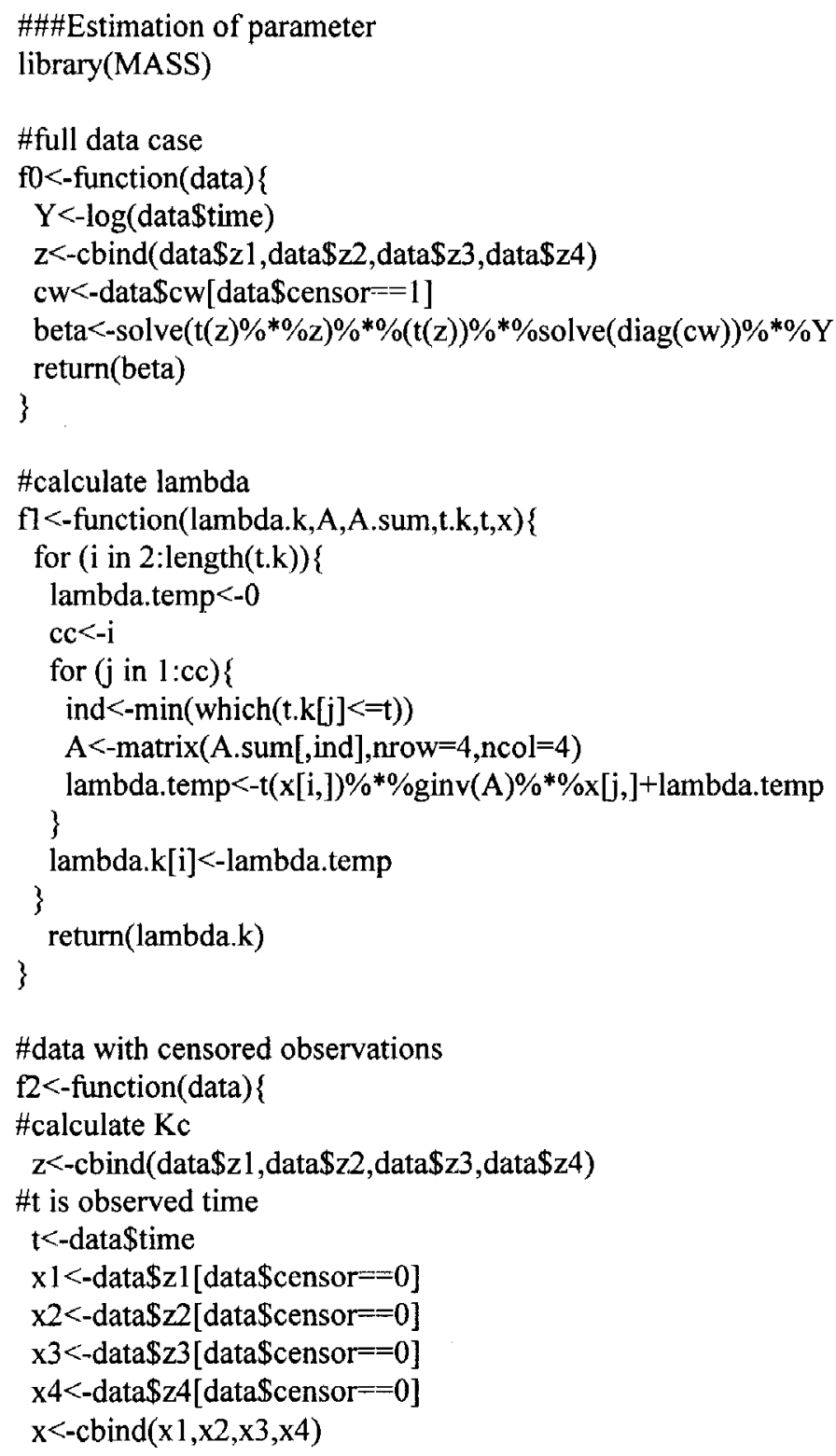


\#t.f is failure time

t.f $<$-data $\$$ time[data $\$$ censor $=1$ ]

\#t.k is time use for reweighting survival function

t.k<-data\$time[data $\$$ censor $=0$ ]

lambda.k<-NULL

A.m $<$-matrix $($ nrow $=4 * 4$, ncol $=$ n)

for (i in $1: n)$ \{

A.m[,i]<-as.numeric $(z[i] \% * \% t,(z[i])$, \}

A.sum<-matrix (nrow $=4 * 4$, ncol $=$ n)

A.sum[,n]<-A.m[,n]

for $(\mathrm{i}$ in $(\mathrm{n}-1): 1)\{$

A.sum $[, \mathrm{i}]<-A . m[, \mathrm{i}]+$ A.sum[,i+1]

\}

ind $<-\min ($ which $(t . k[1]<=t))$

A<-matrix(A.sum[,ind],nrow $=4$, ncol $=4$ )

lambda.k[1]<-t(x[1,])\%*\%ginv(A)\%*\%x[1,]

\# extra step for if only one censored event

ifelse (length(t.k)==1, lambda.k<-lambda.k, lambda.k<-fl(lambda.k,A,A.sum,t.k,t,x))

surv.k<-exp(-lambda.k)

\#add an extra beginning point for time and survival probability of censoring times

t.k<-c(-1,t.k)

surv. $k<-c(1$, surv.k)

Ke $<-$ NULL

for (i in 1:length(t.f)) \{

$\mathrm{Kc}[\mathrm{i}]<$-surv.k[max $($ which $(\mathrm{t} . \mathrm{k}<\mathrm{t.f}[\mathrm{i}]))]$

\}

\#delete extreme case

if (Kc[length $(\mathrm{Kc})]<0.00001) \mathrm{h}<-3$

\#adjust for cluster weighted method

$\mathrm{cw}<$-data $\$ \mathrm{cw}[$ data $\$ \mathrm{censor}==1]$

$\mathrm{Kc}<-\mathrm{Kc} \mathrm{c}^{*} \mathrm{cw}$

\#obtain $Y$ and define $w$

$Y<-\log$ (data\$time[data\$censor $==1]$ )

wl<-data\$zl [data\$censor $==1]$

w2<-data $\$ 2$ [data $\$$ censor $==1$ ]

w3<-data $\$$ z3 [data\$censor $=1$ - 1 ]

w4<-data $\$ \mathrm{z} 4$ [data $\$$ censor $==1]$

$\mathrm{w}<$-cbind(w1,w2,w3,w4)

\#define for sigular case

$\mathrm{B}<-\mathrm{t}(\mathrm{w}) \% * \% \operatorname{ginv}(\operatorname{diag}(\mathrm{Kc})) \% * \% \mathrm{w}$

$\mathrm{h}<-\mathrm{qr}(\mathrm{B}) \$ \mathrm{rank}$

ifelse $(\mathrm{h} !=4$, beta.est $<-\mathrm{c}(999,999,999,999)$, beta.est $<$-solve(B)\%*\%(t(w))\%*\%solve(diag(Kc))\%*\%Y)

return(beta.est)

\}

\#\#\#asymptotic variance

\#adjusted simulation size

11<-which(beta.est[,1]!=999)

$\mathrm{M}<$-length(11)

variance $<$-list $(\mathrm{M})$

vava $<$-matrix $(0$, nrow $=4$, ncol $=4)$

for $(\mathrm{j}$ in $1: M)$ \{ 


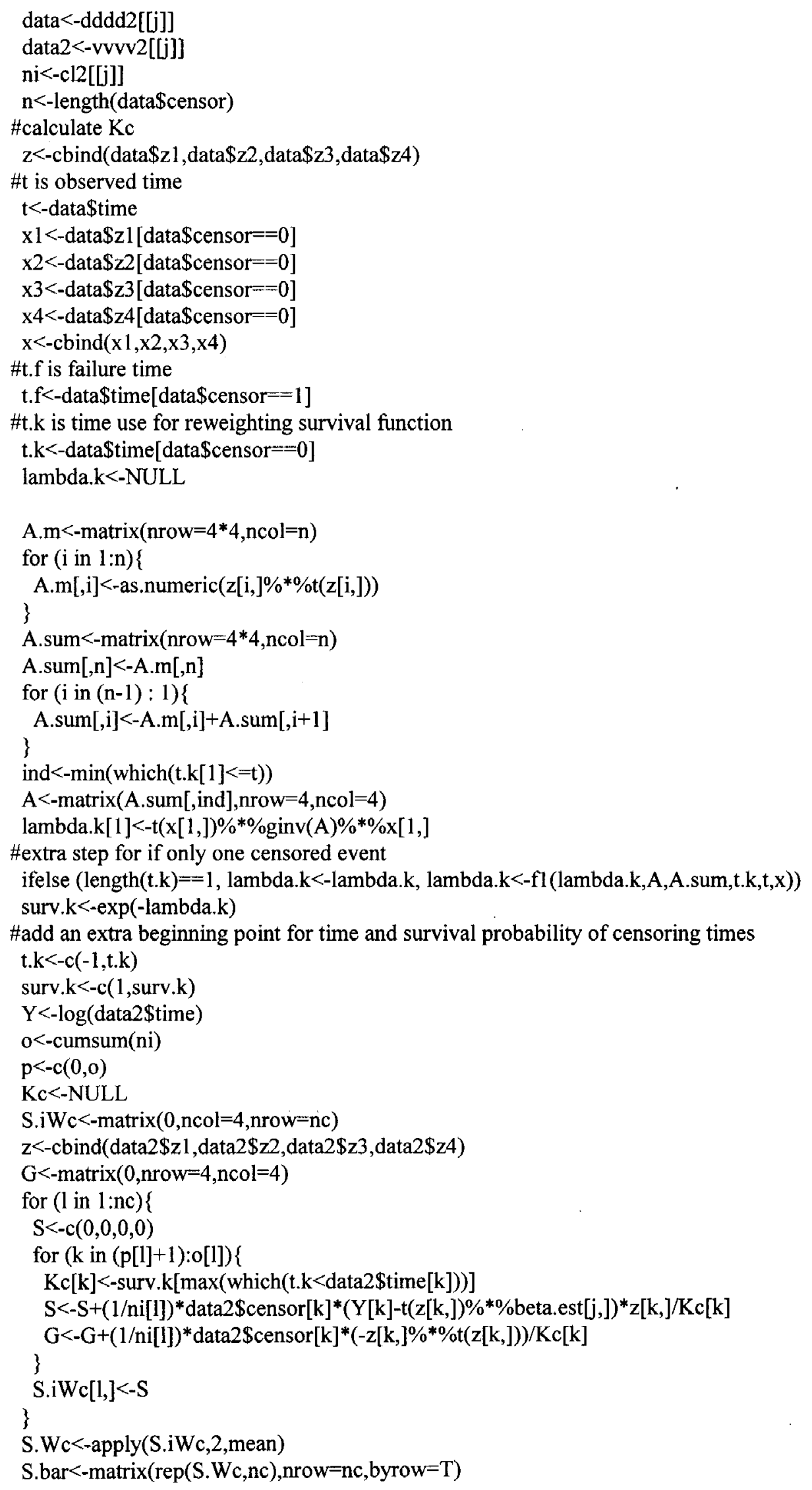




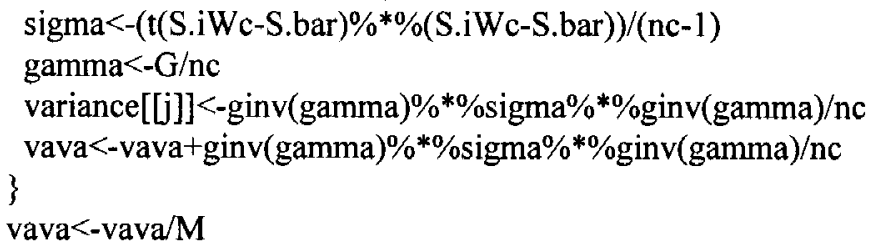

Key $R$ Programs for Comparing Sojourn Time Distributions When Transition Times Are Right Censored

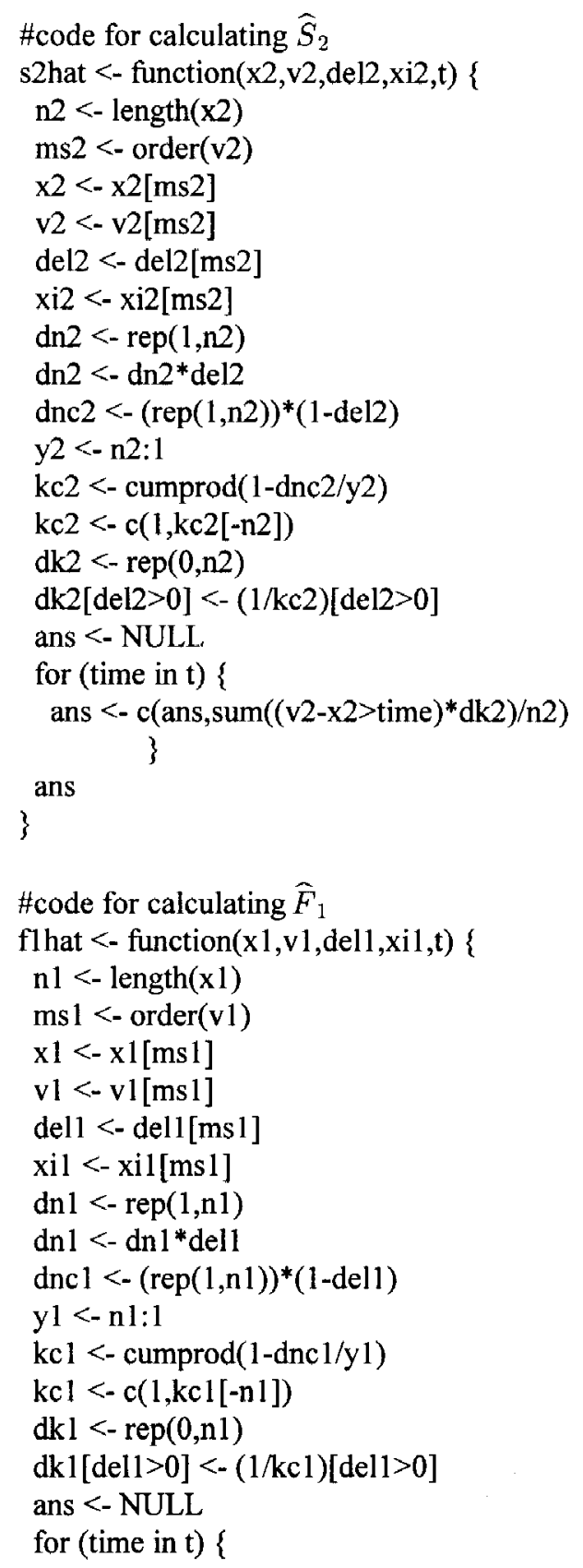




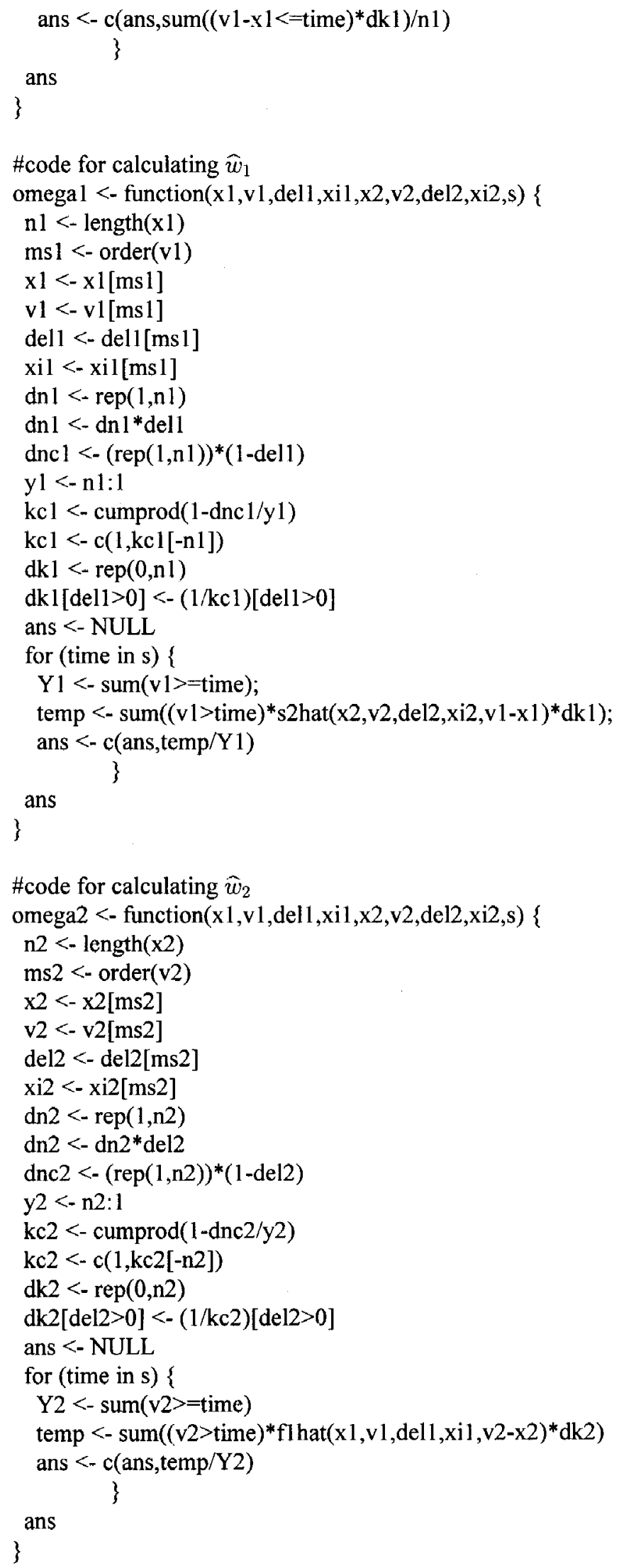




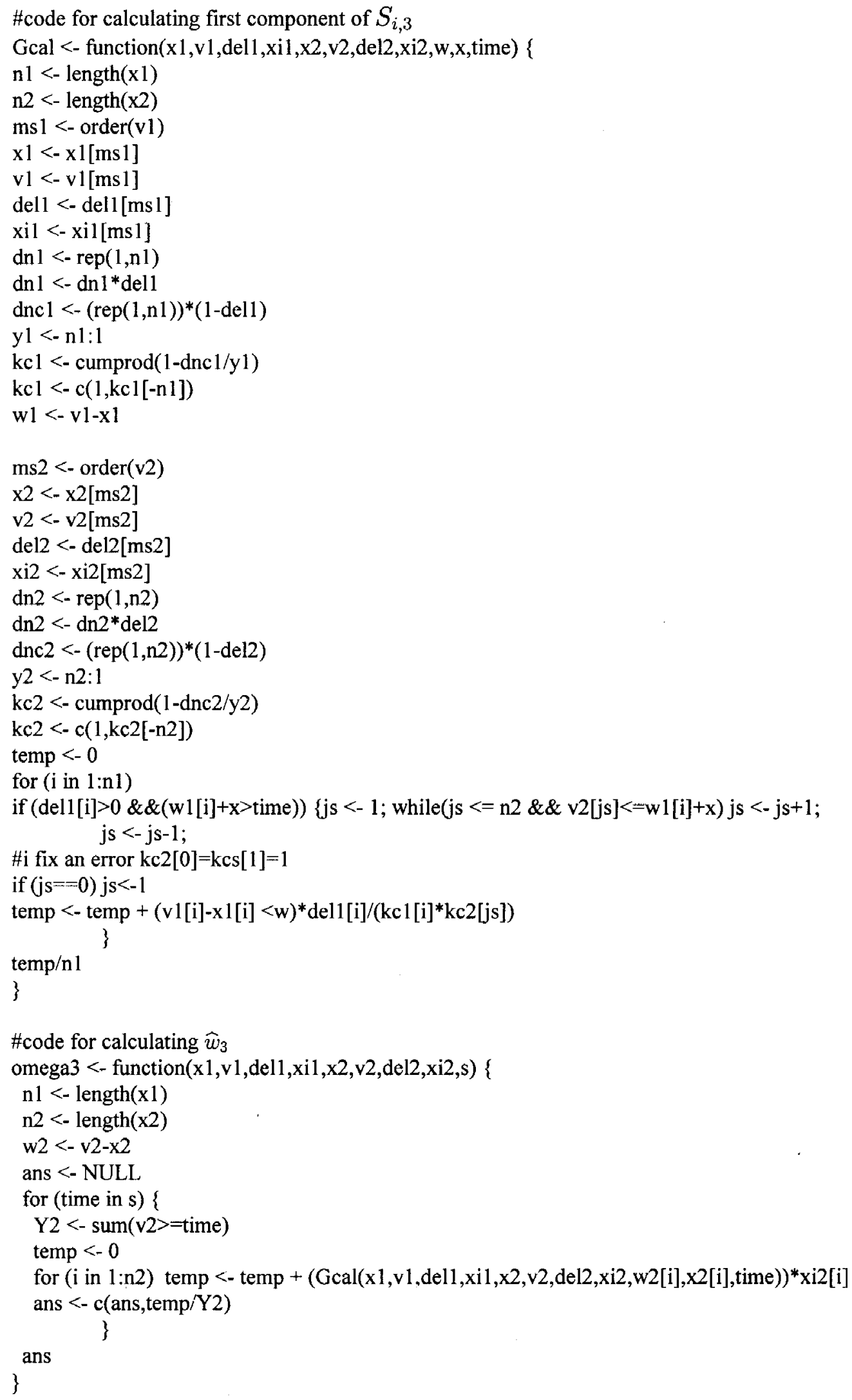




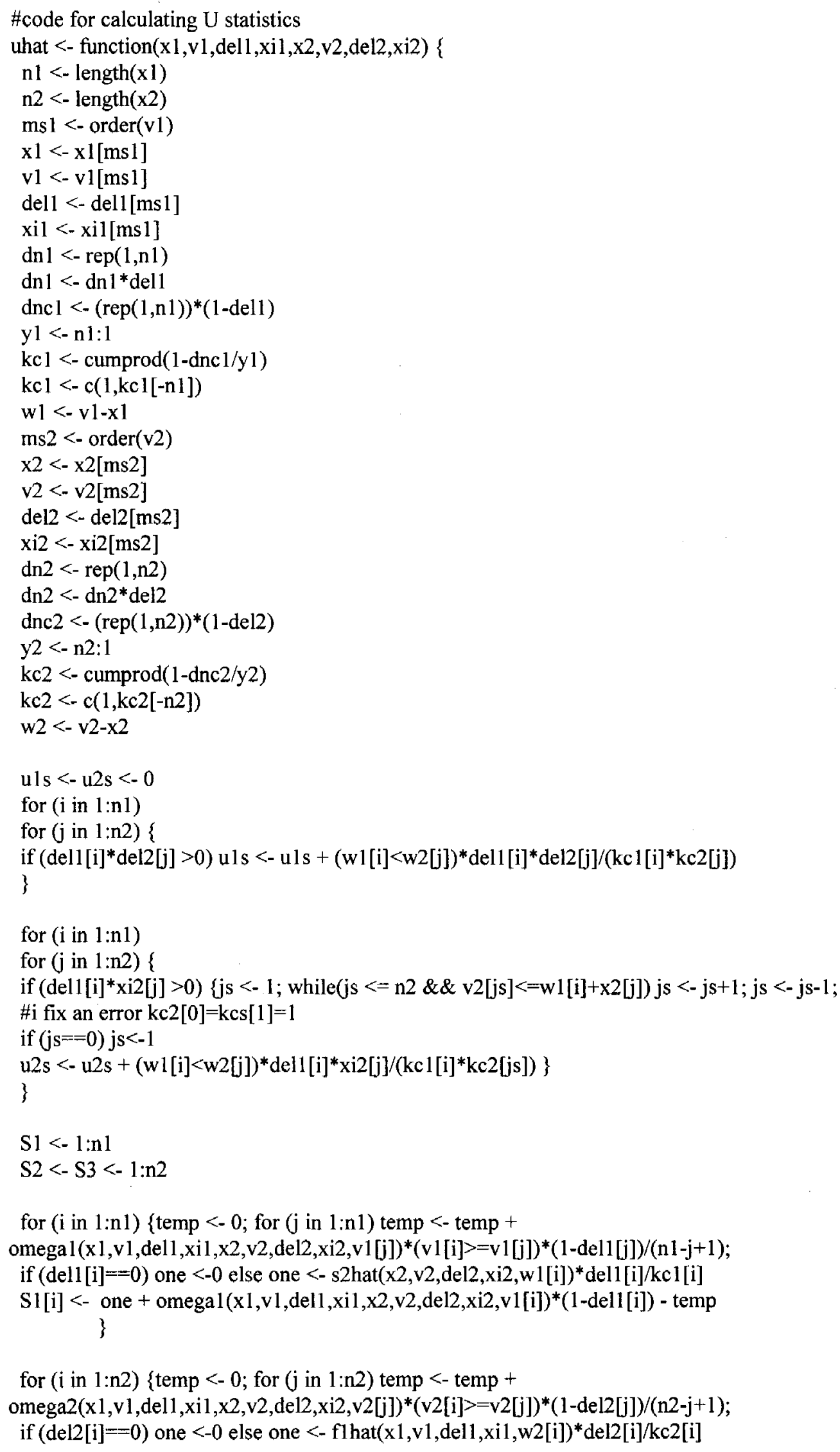




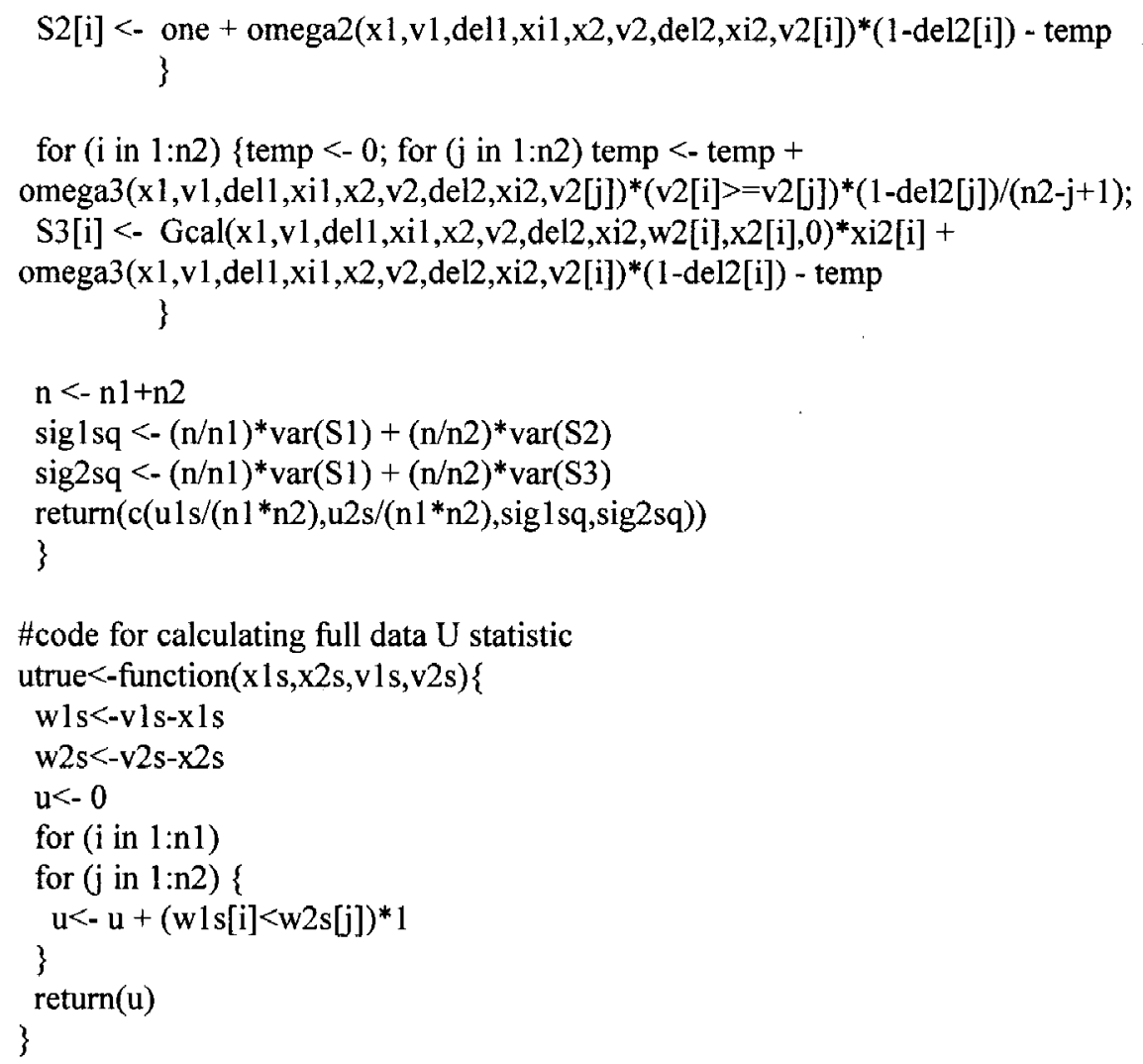




\section{CURRICULUM VITAE}

NAME:

ADDRESS:

EDUCATION

\&TRAINING:
JIE FAN

2809 South Third Street Apt. 5

Louisville, KY 40208

Diploma, Engineering

Shanghai Electric Industry School

1991-1995

B.A., Management

IMI,

1997-1999

MBA, Business Administration

Webster University

2000-2001

MPA, Public Administration

University of Louisville

2003-2005

MS, Biostatistics and Decision Science University of Louisville

2006-2007

PROFESSIONAL SOCIETES:

American Statistical Association (ASA) 


\section{PUBLICATIONS:}

Woodall, C. E., Brock, G. N., Fan, J., Byam, J. A., Scoggins, C. R., McMasters, K. M. and Martin, R. G. (2009). An evaluation of 2537 gastrointestinal stromal tumors for a proposed clinical staging system, Arch. Surg. 144, 670-678.

Datta, S. and Fan, J. Mann-Whitney test for comparing two sojourn time distributions when transition times are right censored. (Submitted)

Fan, J. and Datta, S. Inference for AFT models for clustered survival data with potentially informative cluster size. (Paper in preparation)

\section{PRESENTATIONS:}

Contributed talk. "Mann-Whitney test for comparing waiting time distributions when transition times are right censored". ICSA, Indianapolis, IN, June 2010.

Poster presentation. "Inference for AFT models for clustered survival data with potentially informative cluster size". ENAR, New Orleans, LA, March 2010.

Poster presentation. "Inference for AFT models for clustered survival data with potentially informative cluster size". Research of Louisville, Louisville, KY, November 2009. 